\title{
MANUFACTURE OF SUPERCONDUCTING WIRE
}

\section{PROGRESS REPORT}

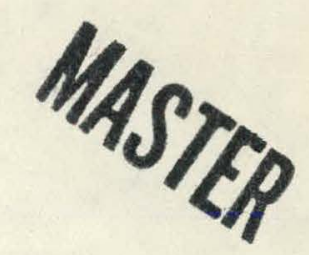

For the period of February 1 to October 15, 1979

Professors B. Avitzur, Y. T. Chou, and S. Talbert and $\mathrm{Mr}$. R. Wu

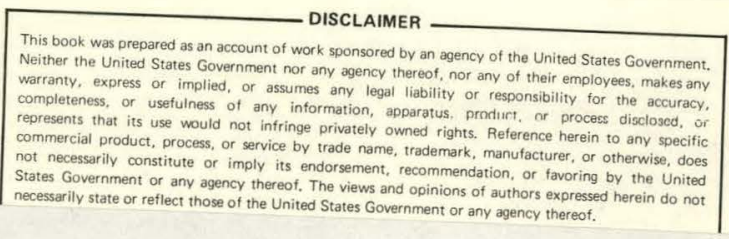

Lehigh University

Bethlehem, Pa. 18015

October 1979

Prepared for

The U. S. Department of Energy

Division of Materials Sciences.

Office of Basic Energy Sciences Under Contract DE-ACO2-79ER 103815 


\section{DISCLAIMER}

This report was prepared as an account of work sponsored by an agency of the United States Government. Neither the United States Government nor any agency Thereof, nor any of their employees, makes any warranty, express or implied, or assumes any legal liability or responsibility for the accuracy, completeness, or usefulness of any information, apparatus, product, or process disclosed, or represents that its use would not infringe privately owned rights. Reference herein to any specific commercial product, process, or service by trade name, trademark, manufacturer, or otherwise does not necessarily constitute or imply its endorsement, recommendation, or favoring by the United States Government or any agency thereof. The views and opinions of authors expressed herein do not necessarily state or reflect those of the United States Government or any agency thereof. 


\section{DISCLAIMER}

Portions of this document may be illegible in electronic image products. Images are produced from the best available original document. 


\section{The following pages are an exact representation of what is in the original document folder.}




\section{NOTICE}

This report was prepared as an account of work sponsored by the United States Government. Neither the United States nor the United States Department of Energy, nor any of their employees, nor any of their contractors, subcontractors, or their employees, makes any warranty, express or implied, or assumes any legal liability or responsibility for the accuracy, completeness, or úsefuiness of any information, apparatus, product or process disclosed or represents that its use would not infringe privately owned rights. 


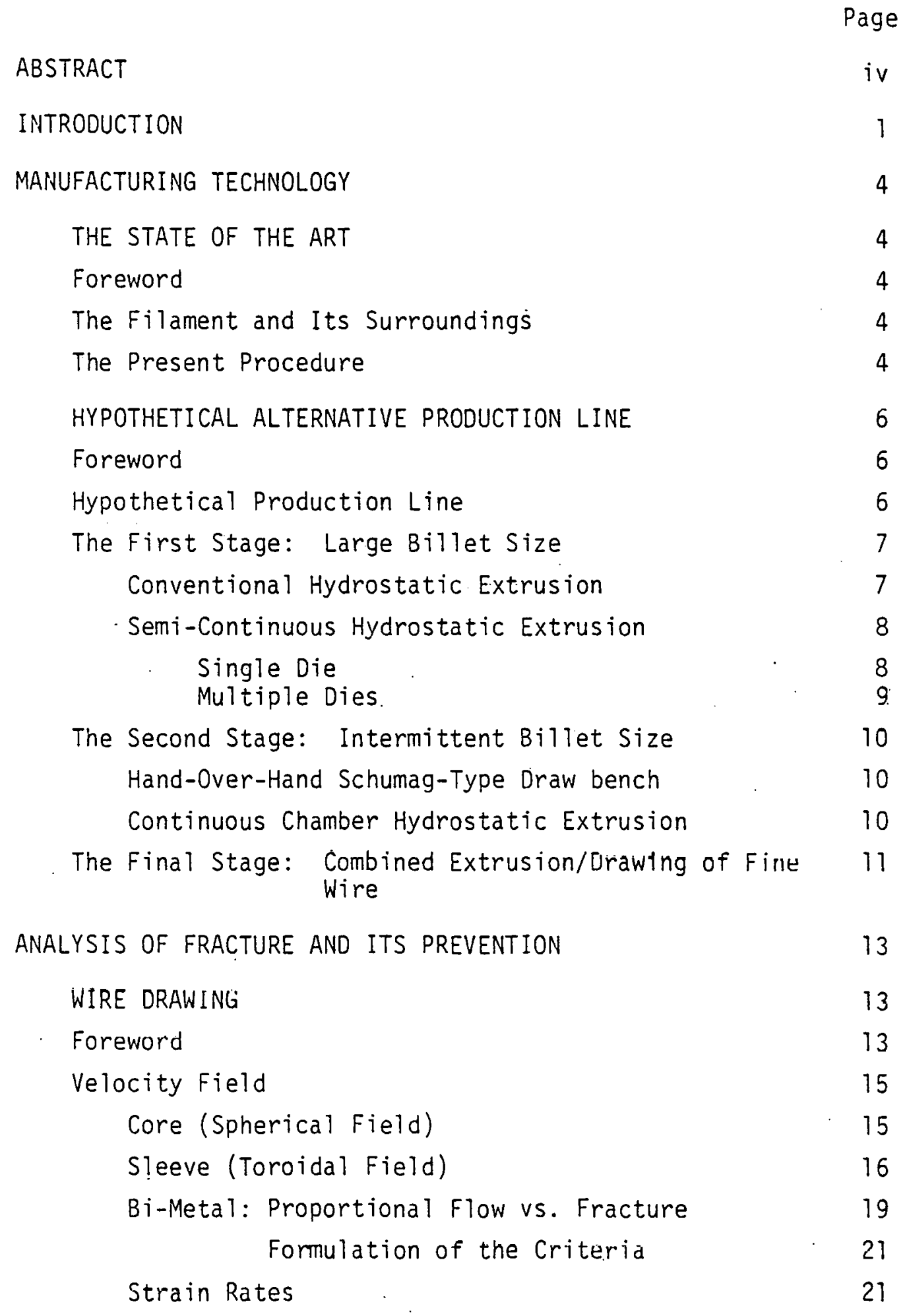


(1) The External Power: $\dot{W}_{e} 25$

(2) The Internal Power of Deformation 25

(3) The Shear Power in the Core: $\dot{W}_{\text {SC }} 27$

(4) The Friction Power along the Bearing 27

(5) The Internal Power of Deformation in the Sleeve: $\dot{w}_{\text {is }}$

(6) The Shear Power on the Interface: 29 $\dot{W}_{\text {interface }}$

(7) The Shear Power in the Sleeve: $\dot{W}_{\text {SS }} \quad 31$

(8) The Friction Power between the Sleeve and the Surface of the Die: $\dot{W}_{f s}$

(9) The Fracture Energy Associated with Core Fracture or Sleeve Fracture: $\dot{W}_{\text {fracture }}$

THE CHARACTERISTICS OF J*; The Contour Lines · 35

$\begin{array}{ll}\text { CRITERIA FOR CORE FRACTURE } & 36\end{array}$

PROPERTIES OF THE SUPERCONDUCTING WIRE 38

REFERENCES $\quad 40$

APPENDIX A AT

APPENDIX B B B B. 


\section{LIST OF FIGURES}

No. Caption

1 BILLET ASSEMBLY: TYPICAL $\mathrm{Nb}_{3} \mathrm{Sn}$ SUPERCONDUCTING WIRE

2 HYPOTHETICAL PRODUCTION LINE

3 PRESSURE TO PRESSURE EXTRUSION

4 SEMI-CONTINUOUS HYDROSTATIC EXTRUSIION

5 MULTISTAGE SEMI-CONTINUOUS EXTRUSION

6. DRAWBENCH

7 CONTINUOUS CHAMBER

8 WIRE SPOOL

9 THE SPHERICAL VELOCITY FIELD FOR THE CORE

10 THE TOROIDAL VELOCITY FIELD FOR THE SLEEVE

11 (a) PROPORTIONAL FLOW OF THE SLEEVE WITH $e=0$

(b) PROPORTIONAL FLOW OF THE SLEEVE WITH $\varepsilon \div 0$

(c) THE EFFECT OF $\varepsilon$ ON SLEEVE THINNING \& THICKENING

12 COMBINED FLOW OF THE SLEEVE AND THE CORE

13 TOTAL POWER VS. ECCENTRICITY FACTOR \& WHEN DEVIATION FACTOR $\varepsilon$ IS ZERO

14 TOTAL POWER vS. DEVIATION FACTOR $\varepsilon$ (at $a=\epsilon_{\text {opt }}{ }^{\prime}$ FOR PROPORTIONAL FLOW

15 TOTAL POWER vs. DEVIATION FACTOR $\varepsilon$ (at $e=e_{\text {opt }}$ ) FOR CORE FRACTURE, ( $a, b)$

$16 J *$ CONTOUR FOR PROPORTIONAL FLOW

$17 \mathrm{~J} *$ CONTOUR FOR CORE FRACTURE

18 CRITERION FOR CORE FRACTURE IN DRAWING
(a) $\frac{\sigma_{O S}}{\sigma_{O C}}$ vs. ${ }^{R_{i} / R_{0}}$ with changing $\alpha$
(b) $\frac{\sigma_{O S}}{\sigma_{O C}}$ vs. ${ }^{R_{i}} / R_{0}$ with Changing $r \%$
(c) $\frac{\sigma_{O S}}{\sigma_{O C}}$ vs. ${ }^{R_{i}} / R_{0}$ with Changing $M$
(d) $\frac{\sigma_{O S}}{\sigma_{O C}}$ vs. ${ }^{R_{i}} / R_{0}$ with Changing $M_{1}$ 


\section{MANUFACTURE OF SUPERCONDUCTING}

WIRE

\section{ABSTRACT}

This report is divided into three major sections. The first section covers aspects of the manufacturing technology with existing and potential processes, and the second deals with the analysis of flow and fracture and the derivation of criteria to prevent failure.

In the first section, typical present day procedure to assemble a billet, extrude and draw it, with intermediate and final anneals, is described. Then an hypothetical production line with potential alternative processes along the entire path from heavy billet to fine wire, is laid out. Several processes at different stages of development, are offered, with the message that their potential must be explored for the development of more reliable product, at a more economical production cost.

In the second section the analysis of core fracture and its prevention during the process of drawing is provided. Treatment of velocity fields for a bi-metallic rod leads to the determination of criteria for the prevention of fracture. The criteria are presented graphically.

The third section of the report describes work on micro properties of superconducting wires. A modification of the bronze method for niobium-tin superconductors is proposed and a preliminary schedule of the testing procedures is outlined. 


\section{INTRODUCTION}

This present progress report covers the results of the first eight months of a multipurpose study proposed to the Division of Material Sciences, Office of Basic Energy Sciences, of the Department of Energy. The major aspects of the proposed study cover the following:

1 Assistance in the development of advanced manufacturing technology for superconducting wire.

2 Development of analys is to assist in understanding failure modes in the production of multifilament superconducting wire (especially during flow through conical converging dies) which occur both during drawing and during extrusion.

3 Study of the properties of superconducting wire and the factors controlling them.

The present report covers progress made on the first two items.

\section{Advanced Manufacturing Technology}

Here, very briefly, standard practice is described. Then, in detail, several alternative methods of rod and wire making are presented. The advantages that each process offers for some production rates and selected range of sizes are identified and, thus, an hypothetical alternative production line is offered. Appendix A provides information in more detail for each of the processes offered, together with some other processes for wire making which are not considered relevant to multifilament superconducting wire.

Some hydrostatic extrusion programs for the evaluation of process parameters and product properties have been initiated, but it is too early to report any results.

\section{Failure Analysis}

In the proposal the mechanics of fracture are to be analyzed, and the effects of temperature rise on property deterioration due to premature diffusion of the tin into the niobium are to be studied. There has been progress in both studies. A preliminary computer program is presently available for the study of temperature rise in each constituent of the composite. The background analytical foundation has not yet been written, and no documentation is presentable at this time. 
In the proposal for the steps to be taken in providing the fracture criteria, the following division into major steps was proposed:

(i) Development of the analytical tools to study fracture through the treatment of central burst defects in an homogeneous material. This stage will provide a new improved criterion for the prevention of central burst in homogeneous materials and refinement of the treatment of fracture problems in general. This step has now been fairly accomplished and several velocity fields that wi1l culminate in a separate manuscript and, hopefully, in papers are now considered.

(ii) Development of fracture criteria for bi-metal drawing and extrusion. This study benefits from the more sophisticated techniques developed during the work on stage (i) and presented in this manuscript in the section entitled 'Analysis of Fracture and its Prevention.' Criteria for core fracture during drawing are offered. The derivation will need a check once more. Further refinements which will lead mainly to a more compact presentation, and to better efficiency in the numerical evaluation, are being made.

Presently the derivation of criteria for sleeve fracture during drawing is in an advanced stage. More work is anticipated in repeating the analysis to derive criteria for core and for sleeve fracture during extrusion of bi-metal rod.

(iii) Extending the applicability of the fracture criteria from bi-metal rod to multifilament superconducting wire.

At present, several manufacturers of superconducting wire are using the old criteria for the prevention of central burst in an homogeneous material with success, for the prevention of fracture in multifilament superconducting wire. The criterion for core fracture in bi-metal rod may serve by tiself as a guide for the prevention of fracture of multifilament wire. Guides for such extension will be sought as the next stage of this phase of the study.

\section{Properties of the Superconducting Wire}

A low temperature property laboratory, with limited capacity, is presently assembled in Lehigh's Department of Metallurgy and Materials Engineering to be used on the present study. The capabilities of the present program are extended through the use of facilities elsewhere. 
Presently, for example, microhardness studies are conducted at the Homer Research Laboratory of Bethlehem Steel. These studies are done to explore the possibility of using microhardness as a measure of the diffusion of tin from the bronze and into the niobium. The report on property studies is deferred to a later date. 
MANUFACTURING TECHNOLOGY

THE STATE OF THE ART

Foreword

Superconducting wire comes in a variety of structures; all of these structures are composites of varying degrees of complexity. A typical superconducting material, $\mathrm{Nb}_{3} \mathrm{Sn}$, is a brittle compound that can hardly be formed into the required geometry. Furthermore, to serve as a superconducting wire for any device, the superconductor $\left(\mathrm{Nb}_{3} \mathrm{Sn}\right)$ must be supported by a matrix of a regular conductor that serves as a heat conductor as well as a heat sink. Thus a typical superconductor wire is a complex structure, as shown in Fig. 1. Note that a single wire of $0.43 \mathrm{~mm}$ in diameter may contain from a few hundred to several hundred thousands of $\mathrm{Nb}_{3} \mathrm{Sn}$ filaments (typically, one hexagonal cable contains $44532-\mu$ diameter filaments in each of six strands; see Ref. 1), each one surrounded by the copper matrix.

Please note that actual assembly of a composite billet is much more complex than Fig. 1. The brass sleeve, for example, may be wrapped in a barrier of tantalum sheet (or in a tantalum sleeve) with an outer layer of copper.

The Filament and Its Surroundings

For best performance it is desired to have numerous filaments of the superconducting $\mathrm{Nb}_{3} \mathrm{Sn}$ of as small a diameter as possible embedded in a matrix (presently copper). The procedure described here calls for the assembly of the intended filaments at larger size and achieving the small size by plastic deformations through extrusion and drawing. The nriginal filaments, in rod form, are made of $\mathrm{Nb}$ which is ductile, and only after the final size is reached are the small-sized Nb filaments infiltrated by $\mathrm{Sn}$ to form the superconducting $\mathrm{Nb}_{3} \mathrm{Sn}$ filament, which is brittle. A typical procedure call is for the assembly of individual $\mathrm{Nb}$ core rods with bronze ( $\mathrm{Cu}-\mathrm{Sn}$ ) sleeve rods. Each core/ sleeve combination starts as separate cylindrical $\mathrm{Nb}$ rod in a bronze cylindrical tube. The bi-metal assembly is drawn through an hexagonal die to form a bi-metal hexagonal rod. These rods are assembled manually (see Fig. 1) into a bronze tube of about $7 " \varnothing$ and the assembly is plugged at both ends.

\section{The Present Procedure}

This assembly is heated and extruded two or more times to form a rod of about $1 / 2^{\prime \prime} \emptyset$. After the extrusion the rod is cold drawn through a succession of dies to its final diameter. Through the entire operation 
care must be taken to assure:

1 Sound product without filament fracture.

2 Avoidance of diffusion of the $S n$ from the bronze into the $\mathrm{Nb}$ filaments.

3 Perfect bonding to be established between the individual bronze hexagonals until all boundaries disappear.

4 Perfect bonding to be established between all individual components of the assembly.

If only a few hundred filaments are required, the original assembly will contain the required number of rods. When tens of thousands or hundreds of thousands of filaments are required, such outcome is usually achieved by drawing the first assembly down to about $1 / 4 " \emptyset$ hexagonal shape which then serves as the rod for a new assembly into the $7 " \emptyset$ tube. The extrusion and drawing are then repeated. Note that typically original 700 rods become 490,000 rods. Smaller numbers of rods can be assembled in one tube, but the reassembly may repeat three times to achieve the large number of filaments.

The assembiy and the number of components are much more complex, but for the present discussion this simplified description will suffice.

When the wire is brought down to size (and twisted) a diffusion anneal is employed to caluse diffusion of the $S n$ from the Cu-Sn matrix into the individual filaments. At this point the filaments are so fine that diffusion distance is small and deep penetration of the $S n$ is feasible. Now the filaments are superconducting and the fact that they became brittle is less disturbing because the reduction in size is completed.

One danger in the presently described sequence relates to the conventional extrusion process. The billet must be heated, and the extrudate reaches even higher temperatures because of the work of deformations it undergoes. Some diffusion of the $S n$ into the $N b$ is experienced during this stage. While a little diffusion may be tolerated, excessive diffusion embrittles the filaments which may, subsequently, fracture at any drawing stage. Minimizing this diffusion, or fully eliminating it, is a stated goal of this project. The study of the temperature rise during extrusion and drawing is now in progress but not included in this progress report. 
The drawing operation results too often in filament fracture and the state of the art in the study of the processing parameters that leads to this fracture is included in the section of this report entitled 'Analysis of Fracture and Its Prevention.' Furthermore, the small reduction per pass, during drawing associated with embrittlement by strain hardening, leads to numerous numbers of passes, intermediate anneals, and excessive handling, and these cause the price of the wire to rise and the reliability of the product to drop.

Alternative procedures, including alternative processes, mostly hydrostatic extrusion to replace portions of the presently established practice, are explored next. The goal is to increase reliability and reduce the price.

\section{HYPOTHETICAL ALTERNATIVE PRODUCTION LINE}

\section{Foreword}

In this section an hypothetical production 1 ine for the production of superconducting wire, starting with the $7^{\text {" }} \emptyset$ billet assembly and continuing down to the fine wire, is described. At each stage along this line alternative processes are presented with their advantages. It is not proposed that the entire alternative procedure should replace the present practices. It is only proposed that the alternative processes for each stage be studied to evaluate their feasibilities. The substitution of the alternative processes will be considered at each individual stage.

A variety of hydrostatic extrusion processes will be explored. It is worthwhile to mention here at the onset that the advantages of hydrostatic extrusion as a process, in providing a better product, have already been verified by numerous studies. It will be shown here that when hydrostatic extrusion is explored, the volume of production, which is small indeed, will dictate the design and price of the equipment. For small volume production, less complex extruders can and should be built to make the process economical.

One may envision continuous transfer from station to station rendering the entire process from assembly of filaments to final wire accomplished on a continuous basis.

Hypothetical Production Line

Figure 2 shows schematically three stages in the reduction of the assembled ( $7^{\prime \prime} \emptyset$ ) billet into final wire size $\left(\sim 0.005^{\prime \prime} \emptyset\right)$. Let these stages be identified as large, medium, and small-sized rod or wire respectively. The stages are: 
1 Reduction of Large-Sized Billet. This stage reduction from $7^{\prime \prime} \bar{\emptyset}$ or $5^{11} \emptyset$ to about half an inch is conventionally accomplished by hot extrusion. In the hypothetical line of Fig. 2 this stage can be accomplished by hot or cold hydrostatic extrusion, by semi-continuous hydrostatic extrusion, or by the semicontinuous process patented as 'Con-Die.'

2 Reduction of Medium Size. Medium size is conventionalTy reduced on a drawbench where limited lengths of straight rod are drawn. The batch-type drawbench operation may be replaced by a semi-continuous drawbench operation, or by the continuous hydrostatic extrusion process as developed by Fuchs at Western Electric.

3 Fine-sized Wire. Fine-sized wire is produced on a wiredrawing machine, the wire passing through numerous dies with small reductions in each die. An alternative presented in Fig. 2 is called the 'hi-draw' process which combines drawing and hydrostatic extrusion.

The above-mentioned processes are described in detail in Chaps. 5 and 7 of Ref. 2('Recent Developments of Continuous Wiremaking Machines' and 'Hydrostatic Extrusion' respectively) and some are covered in Ref.3, which appears as an Appendix to the present report.

The remainder of the description of this section ('Hypothetical Alternative Production Line') is devoted to an evaluation of the alternative individual processes and to recommended further exploration of these alternatives.

The First Stage: Large Billet Size

\section{Conventional Hydrostatic Extrusion}

The reduction of large billet.s to smaller diameter by conventional hydrostatic extrusion is described in Chap. 7 of Ref. 2 and in Fig. 3. The process of hydrostatic extrusion is described in numerous works, represented here by Refs. $4-25$. The major feature of the process, when compared with conventional ram extrusion, is the substitution of liquid under pressure to replace the ram and to force the billet to extrude. In many of the above references and others, in Ref. 26, and through work at the .Institute of Metal Forming at Lehigh University, it has been verified beyond doubt that cold hydrostatic extrusion can provide a more reliable product. Uniformity of the extrudate is higher because smaller die angles can be used and the lower temperature assures against premature diffusion. 
Commercial hydrostatic extrusion equipment, however, is expensive and the operation of conventional commercial hydrostatic extrusion equipment is also more expensive than the operation of a conventional ram extrusion unit. Commercial hydrostatic extrusion units are designed for production rates that are much in excess of any near-future predictions for the need for superconducting wire. The equipment is prohibitively expensive because it is over-designed for the limited volume required in superconducting wire. In the upcoming description of the alternative processes the equipment will be considered for the extrusion of one or a few billets a day, not for 60 billets $/ \mathrm{hr}$. Equipment price, in such case, reduces appreciably and becomes more realistic.

There are a number of equipment manufacturers who may be consulted on the feasibility of bringing the production costs of large billets by conventional hydrostatic extrusion into compatibility with production costs by conventional ram extrusion.

Semi-Continuous Hydrostatic Extrusion

Single Die One of the shortcomings of the processes as described earlier; namely, conventional, pressure-to-pressure, and augmented hydrostatic extrusion, is the finite length of the billet and product. The process of semi-continuous hydrostatic extrusion is designed to enable the processing of a continuous billet into a continous product. As will soon be seen, the processing is presently intermittent.

Seyeral versions of the semi-continuous process were proposed by Pugh, 24 Alexander and Lengyel,27-30 McAllen, 31 and Slater and Green. 32

One implementation of the process as proposed by slater and Green 32 is shown in Fig. 4. A continuous feed stock of straight machined rod enters the extrusion chamber from the back through a clamp. The clamp is grasping the bar when the extrusion pressure rises. The extruded rod is passing through the die of an opening smaller in size than the opening in the clamp at the back of the chamber. Thus, if left to its own devices, the billet will tend to move backward, opposite to the extrusion direction. The larger size of the back of the clamp, at which the pressure is equal to the pressure in the extrusion chamber assures a push on the billet to counter the backward force. Note the low pressure vent in the mid-section of the clamping component. At the completion of the forward stroke the extrusion pressure is released, the clamping action ceases, and the low pressure (vent) serves now to retract the clamp without the billet. Repeated cycling results in an intermittent process for the production of a continuous product. Production speeds, however, are relatively slow and fatigue problems are serious. Work on semi-continuous 
hydrostatic extrusion continues at Imperial College, Department of Mechanical Engineeering, London, England.

Multiple Dies The concept of multistage semi-continuous (hydrostatic) extrusion is described in Austen's U. S. Patent (Ref. 33). Recommended for extrusion from heavy or medium size billets of hard metal, where small reductions are feasible through a single die, a series of dies are stacked together. In finite incremental steps, each die in its turn produces its reduction on a portion of the billet. After each die completes its sequence, the cycle recommences repeatedly to complete the entire billet, or as an endless process. In the following description the sealing and motivation details are omitted. Only the concept is discussed.

Two subsequent dies are shown in Fig. 5. At the beginning of each cycle the second die is at its top position, closest to the first die. The cycle starts as a normal extrusion, hydrostatic or conventional, pushing the billet through the first die. The extrudate, emerging through the first die, pushes the second die away from the top. At this part of the cycle the second die is floating freely.

The extrusion through the first die stops and the workpiece is clamped in position. This clamping can be accomplished by applying an upward force on the second die without changing the push ahead of the first die. When the push on the second die is high enough, the intermediate portion of the billet, between the first and second dies, starts to extrude through the second die. The extrusion through the second die proceeds until the second die reaches its utmost high position closest to the first die. The second die then stops moving and extrusion through the second die ceases. One cycle of a finite length has been completed.

If the force on the second die is relieved and the die is rendered free to float again, extrusion pressure on the entrance side of the first die will start a new identical cycle. Larger reductions can be obtained by stacking more than two dies together. The concept with many dies is the same as with two dies. The equipment and sequencing becomes bulkier. Limit switches or solid state control can make the sequenciny automatic.

Dr. A. R. Austen, who was graduated from Lehigh University and who designed and built some of the present hydrostatic equipment at Lehigh, was consulted on the feasibility of constructing a Con-Die unit for exploratory work. He is willing to collaborate, preferrably with Lehigh, on such a study. Exploratory work can be done on the $2^{\prime \prime}$ to $3^{\prime \prime} \emptyset$ billet, reducing it down to $1 / 2^{\prime \prime} \emptyset$ or $1 / 4 " \emptyset$. The estimated price of a Con-Die unit for the above-mentioned reductions and sizes is approximately at $\$ 100,000$. 
The Second Stage: Intermittent Billet Size

Hand-Over-Hand Schumag-Type Drawbench

The intermediate-sized wire is too long for conventional extrusion and too large in diameter to be drawn on a drawing machine with a drum. This intermediate size, from about $20 \mathrm{~mm} \emptyset$ or $12 \mathrm{~mm} \emptyset$ down is traditionally drawn on a drawbench as a straight rod.

Several passes are introduced and the size of the bed of the drawbench limits the size of the drawn rod.

To overcome the length limitations, the company called 'Schumag' has introduced the hand-over-hand concept. By this design, similar to the climbing of mountains by rope, the workpiece is grasped by two grips. While one is pulling forward the other releases its grip and retracts to the furthest-back position. The role of the two grips is sequenced so that by interrupted forward motion an indefinite length of rod can be forced through the die. Obviously, on exiting the drawbench the rod may be cut into lengths or may proceed directly to the next reducing stage on a continuous basis. In the latter case, the smaller-sized wire may finally be suited for coiling and drawing on a wire-drawing machine.

Continuous Chamber Hydrostatic Extrusion To avert the shortcomings of the continuous viscous drag extrusion, Fuchs 34 developed the continuous segmented-chamber extruder. The continuous chamber is made from matching segments moving in a chainlike manner. (See Fig. 7). Four circumferentially-segmented parts form together a short length of the chamber. Longitudinally, many segments form the length of the chamber which is open on both ends. The segments move together parallel to the axis of symmetry of the chamber. They separate at the exit in urder to get away from the chamber they formed and then return to the entrance side where they meet again to form the chamber with their matching counterparts. A die is set at the exit and a rod is fed at the entrance. The rod is squeezed and dragged by the segmented chamber, moving with it towards the die. To enhance the drag, the rod is coated with plastic material before entering the machine. The rod is machined to close tolerances before coating to fit snugly in the chamber. As the rod reaches the die, the drag produces enough pressure to force extrusion through the die. Very little relative motion is expected between the moving chamber and the rod, so that friction losses are minimized. Pressure in the chamber rises from entrance to die end.

The motivation mechanism to mobilize the chamber components and the matching of the components requires a high degree of precision. Cleanliness must be maintained to avoid gaps between the matching segments, through which fins made from the deforming workpiece may squeeze. Besides being precision made, the mechanism must be highly rigid to produce and 
withstand the high operating pressures and forces.

Preparation of the raw material to enter the machine (dimensioning and coating) and the watchmakers' precision required, combined with massivity demands from the production machine parts and assembly, are the major shortcomings of this machine. These shortcomings are compensated by the compactness of the machine. When serviced the machine can be removed physically from the production line and replaced. The process is classified by the inventor as an hydrostatic extrusion process because the coating of the billet separates it from the chamber.

The Fuchs' continous hydrostatic extrusion machine is presently operative successfully with aluminum wire. Die wear problems still exist when harder material like copper is extruded. The continuous nature of the process, the large reductions achieved per pass, and the uniform deformations, all suggest the feasibility of the process. as an alternative process for the second stage, i.e., the production of the intermediate size of the superconducting wire.

Mr. F. J. Fuchs of Western Electric is willing to collaborate on such a study as is here contemplated. Preliminary contacts for the utilization of the equipment at Western Electric for the intermediatesize reduction of wire, supplied by Airco, were made with Western Electric and with Airco.

The Final Stage: Combined Extrusion/Drawing of Fine Wire

One of the shortcomings of the process of hydrostatic extrusion is the finite length of the extruded billet. Since the process of wire drawing has the advantage of being a continuous process, the problem of overcoming the batch-interrupted nature of hydrostatic extrusion has long been with us.

To overcome the interrupted nature of the process, early design 24 fatures rendering the prncess semi-continuous were offered by Pugh, Alexander et al., 30 and many others. In these early concepts the incoming rod and the product were continuous but feeding the material in and forming through the die were done intermittently in a periodic fashion. Speeds by necessity were fluctuating and very slow. These concepts may find their industrial application in the oroduction of large and medium size rod. For further description of these early generations of equipment, please read the section entitled 'Hydrostatic Extrusion of Billets of Unlimited Length' (pp. 502-521) of Ref. 28. In the following two recent developments processing speed is high. 
When a spool of wire (Fig. 8) is inserted into a chamber, longer runs can be made. Simple spools, as in this picture, can be used, and spinning reels and standing reels have been explored at Battelle 35 and by Pugh8 of the National Engineering Laboratories in Scotland. When the extrusion pressure is augmented by a pull, the Robertson 4 process is provided. Hundreds of thousands of feet of fine wire $(<0.001$ inch), used in the solid state industries, can be spooled and inserted into a $3 / 4$ " bore chamber to provide very long runs between loadings.

When fine wire is extruded by liquid pressure alone, the stick slip phenomenon as described in Sec. 11.3.2 of Ref. 36 is manifested to its extreme. As the wire starts moving i.t picks up speed due to reduction in friction resistance. Runout speeds reach such high levels that the wire tears. It is believed that viscous drag within the chamber causes the tearing of the wire from within the chamber. To prevent runout (and other problems) the augmentation by drawing is adopted as follows.

When the pressure in the chamber is raised to $90 \%$ of the runout pressure, the additional required power is supplied by the pulling tension. The pressure chamber is thus supplying the brute force for the large reduction while the pull supplies only the minute extra required power. The pull, however, supplies the delicate speed control which is lacking in conventional hydrostatic extrusion. If the extrusion pressure were the only powar source, it would be hard to control the extrusion rate because of the compressibility of the liquid. Runout at uncontrolled speed or stick-slip are familiar occurrences during the process of conventional, non-augmented hydrostatic extrusion.

The present description is confined to the case of predetermined speed control by the augmenting draw. The extrusion/drawing speed is the independent parameter, together with the set extrusion pressure, while the drawing tension becomes the dependent parameter. Other scheines not discussed here can be considered in which drawing tension and extrusion pressure are the independent parameters and process speed is the dependent parameter.

As the drawing speed increases, the total drawing-plus-extrusion relative stress may increase, decrease, or remain unchanged, as described in Appendix $A$.

Delicate instrumentation to relate pressure to tension controls is incorporated in the earlier attempts both at NEL in Scotland and by Microwire Corp. of Allentown, Pa. Recently Sumitomo Shipbuilding 37 in Japan developed and introduced to the market production equipment called 'Fluidraw' for copper and gold fine wire. Here the extrusion pressure is kept steady at a value lower than the runout pressure and drawing speed is controlled. No feedback is exercised to control pressure, except that extrusion pressure can be raised or lowered manually as drawing speed is controlled. The prevailing thought at Sumitomo is that closed loop auto- 
matic control at prevailing speeds of $\sim 50 \mathrm{~m} / \mathrm{sec}(4500 \mathrm{ft} / \mathrm{min})$ cannot respond fast enough anyway. Uninterrupted runs of several hours of $2.04 \mathrm{~mm}\left(0.0015^{\prime \prime} \emptyset\right)$ of copper wire are reported at speeds of up to $\sim 50 \mathrm{~m} / \mathrm{sec}(4500 \mathrm{ft} / \mathrm{min})$. For the same size wire in gold lower speeds of $150 \mathrm{~m} / \mathrm{min}$ are reported. The Sumitomo machine is the first production equipment of its sort reported and offered in the market today. Much of their success Sumitomo attributes to their use of low viscosity kerosene which they patented.

With present-day limited chamber size, only very fine wire production is expected to be economically competitive for this process. It should also be noted that very long wire can be made but the process is not a truly continous process.

At the final fine-sized superconducting wire, only very small reductions are made in each pass. The material strain-hardens fast and the smaller the size the more often the required anneal is performed. Because the filaments are small in diameter, every anneal invites premature diffusion and embrittlement of the filament, causing subsequent fracture on further drawing. Hi-draw (also called Fluidraw) offers large reductions, elimination of anneal and excessive handing, and more reliable product.

The price of a Hi-Draw production unit may be up to $\$ 100,000$. The experience of the Institute for Metal Forming at Lehigh in hydrostatic extrusion in general, and the expertise in introducing Hi-Draw, both the theory of the process and as a production tool, may help in exploring this avenue further for the production of superconducting wire. A separate proposal for such a study is available.

\section{ANALYSIS OF FRACTURE AND ITS PREVENTION}

\section{WIRE DRAWING}

\section{Foreword}

Before coming to the successful model of bi-metal flow, several attempted velocity fields have been tried, namely,

1 toroidal sleeve with rigid core motion

2 spherical core and toroidal sleeve with eccentricity factor e alone

3 nonhomogeneous spherical field.

For each of these fields the entire work (similar to that shown in the following report on the toroidal field with two pseudoindependent parameters, $e$ and $\varepsilon$ ) was computed. The components of the velocity were determined, the boundaries of velocity discontinuity calculated, and the 
strain rates field derived. The individual terms of the power components were integrated and the total power optimized. When the criteria for fracture then were obtained and plotted, the characteristics of the obtained solution were studied. Causes of shortcomings of the solutions were traced to original assumptions. The original assumptions were then modified to result in the two factors controlling flow and fracture, namely the eccentricity e and deviation from proportional flow $\varepsilon$. The eccentricity factor e could lead to proportional but nonhomogeneous flow. Nevertheless, as long as the deviation factor $\varepsilon$ is zero, proportional flow with non-zero eccentricity factor $e \neq 0$ results in identical reduction in area (and in elongation) in both core and sleeve. Homogeneous flow as the best known flow through conical converging dies is a special proportional flow obeying the following expressions:

$$
\left.\begin{array}{l}
\dot{u}_{r}=v_{f}\left(\frac{r_{f}}{r}\right)^{2} \cos \theta \\
\dot{u}_{\theta}=\dot{u}_{\phi}=0
\end{array}\right\} .
$$

See Eq.8. 4 of Ref. 36 .

Equations (1) describe a plastic flow directed towards the apex of the cone of the die in the region confined by the surface of the die and two spherical surfaces of velocity discontinuity (with a common center at the apex of the die), those surfaces separating the plastic region between the incoming and outgoing rigid bodies.

Nonhomogeneous but proportional flow may deviate from the flow of Eqs. (1) in several manners. For example, a nonhomogeneous spherical field which contains a small deviation factor $\varepsilon$ is described in the following way:

$$
\left.\begin{array}{l}
\dot{U}_{r}=v_{f}(1+\varepsilon)\left(\frac{r_{f}}{r}\right)^{2} \cos \theta \\
\dot{u}_{\theta}=\dot{U}_{\phi}=0
\end{array}\right\}
$$

This deviation will cause a deviation in the shape of one or both of the surfaces of velocity discontinuity from the spherical shape.

The toroidal field introduces a nonradial component to the velocity field. Such a flow conforms better to realities. The toroidal field 
with a rigid core does not reflect the converging nature of the flow in the core. The introduction of a rigid core produces mathematical convergence problems.

The toroidal field with eccentricity term alone may lead to proportional flow with constant relative wall thickness of the sleeve at entrance and exit. It may also lead to relative thickening or thinning of the wall. Thinning of the sleeve in bi-metal drawing leads directly to core fracture. At first the rate of fracture was arbitrarily determined by the eccentricity alone. At this point it was found that the resulting graph, describing the fracture criteria, varied strongly with changes in the arbitrary relation of fracture rate to the eccentricity factor $e$. This observation led to the introduction of the second parameter $\varepsilon$, controliing the influence of the eccentricity on sleeve thinning and on the rate of fracture. This last two-parametric family of admissible velocity fields is presented in the present manuscript.

The assumptions of the now-abandoned simpler models were necessary rungs in the ladder leading to the present acceptable field. The present two-parametric model contains those features of the previous models which were found useful through working out and examining each and every one of the teachings of the previous models, while eliminating the shortcomings.

\section{VELOCITY FIELD}

\section{CORE (Spherical Field)}

The spherical field is described in Ref. 36, Sec. 8.3, as follows:

"A kinematically admissible velocity field is described in Fig.9. The rod is divided into three regions in which the velocity field is continuous. In zones Ic and IIIC the velocity is uniform and has an axial component only. In zone Ic the volocity is $V_{O C}$, and in zone IIIC the velocity is $V_{f c}$. Because of volume constancy

$V_{\text {oC }}=V_{f C}\left(R_{f i} / R_{j}\right)^{2}$

where $\mathrm{R}_{i}$ is the outer radius of the core before form-
$i$.

and $\quad R_{f j}$ is the outer radius of the core after formind, which will be determined later.

In zone Ic deformation has not yet begun. It includes the -incoming rod, which is separated from the deforming zone IIc. 
by the surface $\Gamma_{2 c}$. Surface $\Gamma_{2 c}$ is spherical, of radius $r_{0}$ with the origin at the apex 0 of the cone of the angle $\alpha_{i}$. Zone IIc is the zone of deformation bounded by the surface of the cone with a cone of an included angle $2 \alpha_{i}$ and two concentric spherical surfaces $\Gamma_{1 c}$ and $\Gamma_{2 c}$. The surface $\Gamma_{2 c}$ is the previously mentioned spherical boundary between zones Ic and IIC. The spherical surface $\Gamma_{1 c}$ of radius $r_{f i}$ with the origin at the apex 0 of the cone, separates zone IIc from the emerging product of zone IIIc. In zone IIc the velocity is directed toward the apex 0 of the cone, with cylindrical symmetry.

"In the spherical coordinate system $(r, \theta, \phi)$, the velocity components for zone IIc are

$$
\left.\begin{array}{c}
\dot{u}_{r c}=-v_{o c} \frac{r_{0 i}^{2}}{r^{2}} \cos \theta \\
\dot{u}_{\theta c}=\dot{u}_{\phi c}=0
\end{array}\right\}
$$

Across the boundaries $\Gamma_{1 c}$ and $\Gamma_{2 c}$ the components of velocity normal to the surfaces ( $\Gamma_{1} ;$ and $\Gamma_{2} c$ ) are continuous. There exist velocity discontinuities parallel to these surfaces.

\section{Sleeve (Toroidal Field)}

The toroidal field was introduced in Ref. 38 for the determination of a criterion for the central burst defect. There, in Ref. 38, the treatment employed some approximations and arbitrary short cuts. Here, the field is first introduced with some changes and with no approximations. The power consumption associated with this field will be presented. First, an exact numerical determination 15 pursued. Then, an analytical expression is derived with linearized velocity field and strain rates field. Each expression in the analys is is linearized.

Figure 10 shows the velocity field of the sleeve. Similar to that in the core, there are rigid flow zones $\mathrm{I}_{S}$ and IIIs and the deformation zone II $_{S}$.

In zone IIs the toroidal coordinate system is used. The circle (0) at the distance e from the axis of symmetry is an origin. This e will be named as 'eccentricity factor' from here on. The radial dis- 
tance from 0 ;is $r$, varying from $r_{f s}^{*}$ on $\Gamma_{\text {js }}$ to $r_{\text {os }}^{*}$ on $\Gamma_{2 s}$ ( $_{\text {fs }}^{*}$ and $r_{0}^{*}$ can be functions of the angular position; they will be determined later). The angular position $\theta$ varies from $\alpha_{i}$ on the inner conical surface $\Gamma_{c s}$ to $\alpha$ on the conical surface of the die $\Gamma_{35}$. Please note that in Ref. $37 \alpha_{i}$ was zero while in the present field ${ }^{3 s}$ $\alpha_{i}>0$. The direction of $\theta$ is normal to the direction of $r$. Normal to these two axes $(r$ and $\theta$ ) is the $\phi$-axis. Axial symmetry exists with respect to $\phi$. obey

In this toroidal coordinate system, the velocity is assumed to

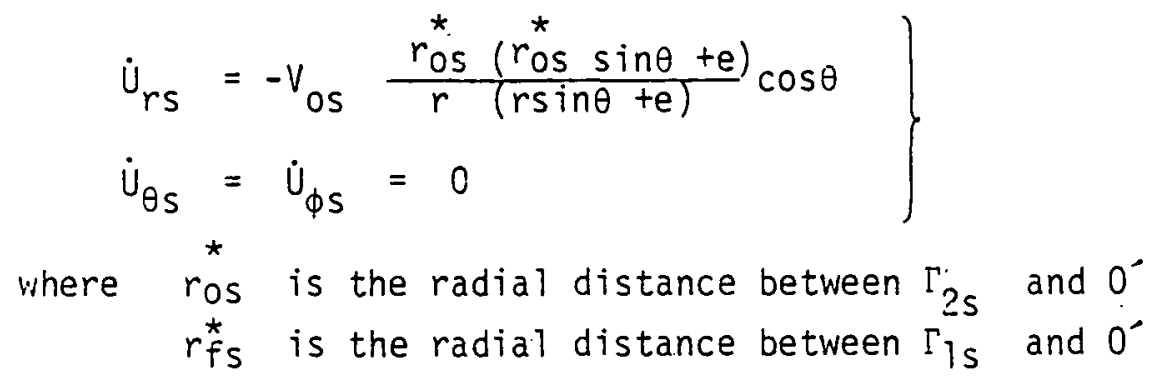

It is noted here that the deformation zone IIs is bounded by surfaces $\Gamma_{3 s}, \Gamma_{2 s}, \Gamma_{C S}$ and $\Gamma_{1 S^{\prime}} . \Gamma_{3 s}$ is the outer conical surface of the sleeve and is predetermined by the semicone angle of the die $\alpha$. For simplicity, the surface of discontinuity $\Gamma_{2 s}$ is assumed to be a toroidal surface The radial distance $r_{0}^{\star}$ s of this surface from the toroidal center $O^{-}$depends only on the eccentricity factor e. Mathematically, this means

$$
r_{O S}^{*}=r_{O S}=\frac{R_{0}-e}{\sin \alpha} \text {, }
$$

which is independent of the angular position $\theta$. The inner conical surface of the sleeve $\Gamma_{C S}$ is also determined through the value of the eccentricity factor e. The semicone angle $\alpha_{j}$ of the surface $\Gamma_{r s}$ is thus a function of e. From the proposed geometry of Fig. 10, we find that

$$
\alpha_{i}=\sin ^{-1}\left\{\frac{R_{i}-e}{R_{0}-e} \sin \alpha\right\}
$$

The value of e itself is pseudoindependent, namely, the value is arbitrary during the evaluation of the strains and power consumptions and is, finally, determined by the concept of minimum energy. (See Ref. 39 for detailed discussion on "limit analysis," the "upper bound approach" and the concept of minimum energy.) 
When proportional flow prevails in the process, it states that the ratio of the inner to outer radius of the sleeve after deformation remains the same as before the deformations, namely,

$$
\frac{R_{f i}}{R_{f}}=\frac{R_{j}}{R_{0}}
$$

where $R_{f}$ is the outer radius of the sleeve after deformation.

In Fig. lla a special case of proportional deformations, namely, homogeneous deformations with a spherical flow $(e=0)$, is described. Even when efo and a toroidal flow exists (see Fig. 11b), it still may be proportional, so that $R_{f i}$ is determined through Eq. (8) and is not a function of the eccentricity (e). Under this circumstance, the surface $\Gamma_{2}$ is toroidal (by our choice) and the angle $\alpha_{i}$ is defined by Eq. (7). The-surface. $\Gamma_{15}$ is not toroidal (if eło) and is determined subsequentiy.

In order to render the exit inner radius $R_{f i}$ a variable and permit the flow to become nonproportional, the deviation factor $\varepsilon$ was introduced as a second pseudoindependent parameter. Both the inner radius $R_{f i}$ and the surface $\Gamma_{\text {s }}$ are then functions of $\varepsilon$ and also of $e$. Since the introduction of the deviation factor $\varepsilon$ will introduce thinning or thickening of the emerging sleeve, it will also introduce a change in the ratio of exit to incoming sleeve velocity.

rrom the established geometry, it is found that the relationship between the incoming velocity $V_{O S}$ of the rigid zone Is and the exit velocity $V_{f s}$ of the outgoing rigid zone IIIs depends on pseudoindependent parameters $e$ and $\varepsilon$. We define this relationship as

$$
\frac{V_{u S}}{V_{f S}} \equiv\left(\frac{R_{f}}{R_{0}}\right)^{2}\left(1-2 \varepsilon \frac{e}{R_{0}}\right)
$$

Whenever $e$ or $\varepsilon$ is equal to zero, this relationship dictates the proporportional flow coidition. For nonzero e and $\varepsilon$ the variations in Rfi and the surface $\Gamma_{1}$ are illustrated in Fin. 11c. In this sigure, when $e \varepsilon>0$ the ratio of exit velocity to entrance velocity will be larger than that for the proportional flow [according to Eq. (9)]. This will lead to the thinning of the sleeve. The surface $\Gamma 1.5$ thus moves to the left side of the proportional position (which is designated as $\Gamma_{i s}$ ); $R_{f j}$, therefore, increases to Rfi. When ee $<_{11} 0$, changes of $\Gamma_{i s}$ and $R_{f i}$ will be reversed. They are shown as $\Gamma_{i s}^{\prime \prime}$ and $R_{f i}^{\prime \prime}$ in Fig. $11 \mathrm{c}$. 
Based on volume constancy and the defined velocity field, the radial distance $r$ fs of $\Gamma_{1 s}$ from $O^{\prime}$ can be obtained as

$$
r_{f s}^{*}(\theta, e, \varepsilon)=-\frac{e}{\sin \theta}+\frac{R_{0}}{\sin \theta} \sqrt{\frac{V_{o s}}{V_{f s}}\left[\left\langle\frac{\sin \theta}{\sin \alpha}+\frac{e}{R_{0}}\left(1-\frac{\sin \theta}{\sin \alpha}\right)\right\rangle^{2}-1\right]+\left(\frac{R_{f}}{R_{0}}\right)^{2}}
$$

where the ratio $V_{0 s} / V_{f s}$ is defined by Eq. (9).

(The detail derivations are deferred. to Appendix B.)

The dimension of $R_{f i}$ is, therefore, found to be

$$
\begin{aligned}
R_{f i} & =\left[\left(r_{f s}^{*}\right) \theta=\alpha_{j} \sin \alpha_{i}+e\right] \\
& \left.=R_{0}\left\{\left.\frac{V_{o s}}{V_{f s}}\right|_{-} ^{-}-\frac{\sin \alpha_{j}}{\sin \alpha}+\frac{e}{R_{0}}\left(1-\frac{\sin \alpha_{j}}{\sin \alpha}\right) j^{2}-1\right]+\left(\frac{R_{f}}{R_{0}}\right)^{2}\right\}^{\frac{1}{2}}
\end{aligned}
$$

\section{Bi-Meta1s: Proportional Flow vs. Fracture}

When two dissimilar metals, one of cylindrical shape called the core with the other surrounding. the core and serving as the sleeve, are combined with tight interface to undergo the drawing process, the following situations may. occur:

1 proportional flow with no fracture

2 defective flow with fracture of the core

3 defective flow with fracture of the sleeve.

In this co-drawing process (see Fig. 12), the plastic flow in the core and in the sleeve is well governed, respectively, by the spherical and the toroidal velocity fields proposed in the previous two sections.

When proportional flow is expected, the core and the sleeve must undergo identical elongation. The resulting deformation could be either homogeneous or nonhomogeneous as mentioned in the foreword. In the homogeneous proportional flow situation, the following two conditions must be obeyed:

$$
\begin{array}{ll}
\frac{R_{f i}}{R_{f}}=\frac{R_{i}}{R_{0}} \\
e=0 & \text { and } \varepsilon=0
\end{array}
$$


For nonhomogeneous proportional flow, the necessary conditions will be the same, except that e should be any value but zero.

If the core undergoes less deformation than the sleeve, the core elongates less than the sleeve and thus the sleeve exerts tensile load on the core and, sooner or later, the core fractures. Mathematically, when the core fractures,

$$
\frac{R_{f i}}{R_{f}}>\frac{R_{i}}{R_{0}}
$$

When the sleeve undergoes less deformation than the core, the sleeve elongates less than the core and the core pulls the sleeve to fracture. Mathematically, when the sleeve fractures,

$$
\frac{R_{f i}}{R_{f}}<\frac{R_{i}}{R_{0}}
$$

For the toroidal field, Eq. (13) means that when core fracture is expected, $e \varepsilon>0$, and Eq. (14) means that when sleeve fracture is expected, ee $<0$.

Experience with the previous velocity field indicates that when core fracture is expected, it occurs along the $\Gamma_{1 c}$ surface. Similarly, sleeve fracture occurs along the $\Gamma_{i s}$ surface.

Whether or not fracture occurs, the following Eqs. (15) to (17) are valid:

1 The entrance velocities of sleeve and core are identical:

$$
\left.\begin{array}{rl}
V_{O C} & =V_{O S}=V_{0} \\
V_{O C} & =\frac{V_{O S}}{V_{O}}=1
\end{array}\right\}
$$

2 For zone IIc of the core,

$$
\frac{V_{f c}}{V_{O C}}=\frac{V_{f c}}{V_{Q}}=\left(\frac{R_{j}}{R_{f i}}\right)^{?}
$$


3 And for zone IIs of the sleeve,

$$
\frac{V_{o s}}{V_{f s}}=\frac{V_{0}}{V_{f s}}=\left(\frac{R_{f}}{R_{0}}\right)^{2} \quad\left(1-2 \varepsilon \frac{e}{R_{0}}\right)
$$

\section{Formulation of the Criteria}

The failures in co-drawing mentioned above, core fracture and sleeve fracture, result from the employment of improper combinations of the process variables including percent reduction in area, semicone angle of the die, the length of the bearing of the die, friction, relative size of the core (ratio of the size of the core to the sleeve), relative strength and properties of the core (ratio of the flow stress of the core to that of the sleeve), and the prescribed body tractions, namely, back tension in drawing. Formulation of the criteria is equivalent to determining the domains of the process variables where in core fracture or sleeve fracture or no fracture are expected. We will use the upper-bound analysis to determine the power expenditures involved in co-drawing. For each particular combination of process variables, the power required for flow with core fracture, for flow with sleeve fracture, and for proportional flow can be derived separately. The principle of minimum power states that for each combination of process parameters the flow that consumes the least power will prevail for that particular combination of process variables. Following this principle, the boundaries of the domains of core fracture, sleeve fracture, and no fracture can be found explicitly to establish the criteria.

The velocity field (core and sleeve) as determined by Eqs. (4) and (5) provides for the calculation of the total power requirements (as a function of $e$ and $\varepsilon$ ) and for the determination of the optimal values of $e$ and $\varepsilon$ that minimize this power.

These calculations initiate with the evaluation of the strain rates values as is done in the next section, this leading to the evaluation of the velocity discontinuities and determination of internal power of deformations and shear and friction losses as is done subsequently.

\section{Strain Rates}

In upper-bound analysis, the power to overcome the resistance of the material to deformation is estimated through the integration of the product of the strain rate and the yield stress required over the entire 
deformation volume. Therefore, it is necessary to derive the strain rates fields for the proposed spherical and toroidal fields. First, we will summarize the spherical and toroidal velocity fields as follows:

Spherical velocity field,

$$
\left.\begin{array}{l}
\dot{u}_{r c}=-v_{O C}\left(\frac{r_{0 i}}{r}\right)^{2} \cos \theta \\
\dot{U}_{\theta C}=\dot{u}_{\phi c}=0
\end{array}\right\}
$$

Toroidal velocity field,

$$
\left.\begin{array}{l}
\dot{U}_{r S}=-v_{\text {os }} \frac{r_{O S}\left(r_{0 S} \cdot \sin \theta+e\right)}{r(r \cdot \sin \theta+e)} \cos \theta \\
\dot{U}_{\theta S}=\dot{u}_{\phi S}=0
\end{array}\right\}
$$

The strain rates in the spherical field as functions of velocity components are (Ref. 40):

$$
\left.\begin{array}{l}
\dot{\varepsilon}_{r r}=\frac{\partial \dot{U} r}{\partial r} \quad \dot{\varepsilon}_{\theta \theta}=\frac{1}{r} \frac{\partial \dot{U} \theta}{\partial \theta}+\frac{\dot{U}_{r}}{r} \\
\dot{\varepsilon}_{\phi \phi}=\frac{1}{r \sin \theta} \frac{\partial \dot{U} \phi}{\partial \phi}+\frac{\dot{U}_{r}}{r}+\frac{\dot{U} \theta}{r} \cot \theta \\
\dot{\varepsilon}_{r \theta}=\frac{1}{2}\left(\frac{\partial \dot{U} \theta}{\partial r}-\frac{\dot{U} \theta}{r}+\frac{1}{r} \frac{\partial \dot{U}_{r}}{\partial \theta}\right) \\
\dot{\varepsilon}_{\theta \phi}=\frac{1}{2}\left(\frac{1}{r \sin \theta} \frac{\partial \dot{U} \theta}{\partial \phi}+\frac{1}{r} \frac{\partial \dot{U} \phi}{\partial \theta}-\frac{\cot \theta}{r} \dot{U}_{\phi}\right) \\
\dot{\varepsilon}_{\phi r}=\frac{1}{2}\left(\frac{\partial \dot{U} \phi}{\partial r}-\frac{\dot{U}_{\phi}}{r}+\frac{1}{r \sin \theta} \frac{\partial \dot{U} r}{\partial \phi}\right)
\end{array}\right\}
$$

In the toroidal field, according to Sokolnikoff's [41] Eqs. (48.7) and (48.9), the strain rates are: 


$$
\left.\begin{array}{l}
\dot{\varepsilon}_{i j}=\frac{\partial}{\partial \alpha_{i}} \frac{\ddot{U}_{i}}{\sqrt{g_{i j}}}+\frac{1}{2 g_{i j}} \sum_{k=1}^{3} \frac{\partial g_{i j}}{\partial \alpha_{k}} \frac{\dot{U} k}{\sqrt{g_{k k}}} \text { if } i=j \\
\dot{\varepsilon}_{i j}=\frac{1}{2 \dot{g}_{i j} g_{j j}}\left[g_{i i} \frac{\partial}{\partial \alpha_{j}}\left(\frac{\dot{U}_{i}}{\sqrt{g_{i j}}}\right)+g_{j j} \frac{\partial}{\partial \alpha_{i}}\left(\frac{\dot{U} j}{\sqrt{g_{j j}}}\right) \text { if } i \neq i\right.
\end{array}\right\}
$$

where

$$
g_{r r}=1, g_{\theta \theta}^{\prime}=r^{2} \text { and } g_{\phi \phi}^{\prime}=(r \sin \theta+e)^{2}
$$

for the present toroidal field.

Calculating the strain rates, substituting $\dot{U}_{\theta}=\dot{U}_{\phi}=0$,

$$
\begin{aligned}
& \dot{\varepsilon}_{r r}=\frac{\partial \dot{u}_{r}}{\partial r} \\
& \dot{\varepsilon}_{\theta \theta}=\frac{\dot{U} r}{r} \\
& \dot{\varepsilon}_{\phi \phi}=\frac{\dot{U}_{r} \sin \theta}{r \sin \theta+e} \\
& \dot{\varepsilon}_{r \theta}=\frac{1}{2 r} \frac{\partial \dot{U} r}{\partial \theta} \\
& \dot{\varepsilon}_{\theta \phi}=\dot{\varepsilon} r \phi=0
\end{aligned}
$$

From Eqs. (4) and (18), the strain rates for the spherical field can be calculated. They are

$$
\begin{aligned}
& \dot{\varepsilon}_{r r}=-2 \dot{\varepsilon}_{\theta \theta}=-2 \dot{\varepsilon}_{\phi \phi}=2 v_{o c} r_{o i}^{2} \frac{\cos \theta}{r^{3}} \\
& \dot{\varepsilon}_{r \theta}=\frac{1}{2} v_{0 c r o i}{ }^{2} \frac{\sin \theta}{r^{3}} \\
& \dot{\varepsilon}_{\theta \phi}=\dot{\varepsilon}_{r \phi}=0
\end{aligned}
$$

Similarly, Eqs. (5) and (2.0) give us the strain rates for toroidal field: 


$$
\begin{aligned}
& \dot{\varepsilon}_{r r}=v_{0 s} \frac{r_{0 s}\left(r_{0 s} \sin \theta+e\right)(2 r \sin \theta+e)}{r^{2}(r \sin \theta+e)^{2}} \cos \theta \\
& \dot{\varepsilon}_{\theta \theta}=-v_{0 s} \frac{r_{0 s}\left(r_{0 s} \sin \theta+e\right)}{r^{2}(r \sin \theta+e)} \cos \theta \\
& \dot{\varepsilon}_{\phi \phi}=-v_{\text {os }} \frac{r_{0 s}\left(r_{0 s} \sin \theta+e\right)}{r(r \sin \theta+e)^{2}} \sin \theta \cos \theta \\
& \dot{\varepsilon}_{r \theta}=\frac{1}{2} \cdot v_{0 s} \frac{\left.r_{0 s}[r \sin \theta+e)\left(r_{0 s} \sin \theta+e\right) \sin \theta-e\left(r_{0 s}-r\right) \cos ^{2} \theta\right]}{r^{2}(r \sin \theta+e)^{2}} \\
& \dot{\varepsilon}_{\theta \phi}=\dot{\varepsilon}_{r \phi}=0
\end{aligned}
$$

The above strain rates for the toroidal field, when linearized with respect to the eccentricity factor $e$, become

$$
\begin{aligned}
& \dot{\varepsilon}_{r r}=v_{o s} \bar{r}_{o s}^{2} \frac{\cos \theta}{r 3}\left\{2-\frac{e}{R_{0}}\left[4+\left(3 \frac{\overline{r_{0 S}}}{r}-2\right) \frac{\sin \alpha}{\sin \theta}\right)\right\} \\
& \dot{\varepsilon}_{\theta \theta}=-v_{o s} \bar{r}_{\text {os }}^{2} \frac{\cos \theta}{r^{3}}\left\{1-\frac{e}{R_{0}}\left[2+\left(\frac{\overline{r_{o s}}}{r}-1\right) \frac{\sin \alpha}{\sin \theta}\right]\right\} \\
& \dot{\varepsilon}_{\phi \phi}=-v_{o s} \bar{r}_{o s}^{2} \frac{\cos \theta}{r^{3}}\left\{1-\frac{e}{R_{0}}\left[2+\left(2 \frac{\overline{r_{\sigma s}}}{r}-1\right) \frac{\sin \alpha}{\sin \theta}\right]\right\} \\
& \dot{\varepsilon}_{r \theta}=v_{\text {os }} \overline{r_{\text {os }}^{2}} \frac{\sin \theta}{2 r^{3}}\left\{1-\frac{e}{R_{0}}\left[2+\csc ^{2} \theta\left(\frac{r_{0 S}}{r}-1\right) \frac{\sin \alpha}{\sin \theta}\right)\right\} \\
& \dot{c}_{\dot{\theta} \phi}=\dot{\varepsilon}_{r \phi}=0
\end{aligned}
$$

where $\bar{r}_{\text {os }}=\frac{R_{0}}{\sin \alpha}$

$$
r_{\text {os }}=\frac{R_{0-e}}{\sin \alpha}
$$

The compressibility equation $\dot{\varepsilon}_{r r}+\dot{\varepsilon}_{\theta \theta}+\dot{\varepsilon}_{\phi \phi}=0$ is still
held in linearized expressions. 


\section{POWER CONSUMPTION}

The upper bound on power consumption during co-drawing of sleeve and core composed of two dissimilar materials is given by

$$
\begin{aligned}
J^{*}= & \dot{W}_{e}+\dot{W}_{i c}+\dot{W}_{s c}+\dot{W}_{f}+\dot{W}_{i s}+\dot{W}_{\text {interface }}+\dot{W}_{s s}+\dot{W}_{f s} \\
& \left.+\dot{W}_{\text {fracture }}\right) / \pi \sigma_{o c} V_{f s} R_{f}^{2}
\end{aligned}
$$

where the definition of the individual power terms follows:

\section{(1) The External Power : $\dot{W}_{e}$}

The external power $W_{e}$ is associated with the prescribed body tractions. For drawing, the prescribed body traction is the back tension $\sigma_{x b}$, and the external power becomes

$$
\begin{aligned}
\dot{W}_{e}=\dot{W}_{b} & =\pi\left\{V_{o c} R_{i}^{2}+V_{o s}\left(R_{0}{ }^{2}-R_{i}{ }^{2}\right)\right\} \sigma_{x b} \\
& =\pi V_{0} R_{0}{ }^{2} \sigma_{x b}
\end{aligned}
$$

\section{(2) The Internal Power of Deformation in the core : $\dot{w}_{\text {ic }}$}

For the core, the simple spherical field of Eq. (4) was assumed through this entire work. With this spherical field, the internal power of deformation in the core, by Eq. (8.11) of Ref. 36, becomes

$$
\begin{aligned}
\dot{w}_{i c} & =\frac{2}{\sqrt{3}} \sigma_{O C} \int_{0}^{\alpha_{i}} \int_{r_{f i}}^{r_{0 i}} v_{o c} r_{o i}{ }^{2} \frac{1}{r^{3}} \sqrt{3 \cos ^{2} \theta+\frac{1}{4} \sin ^{2} \theta}(2 \pi r \sin \theta r d \theta d r) \\
& =2 \pi \sigma_{O C} V_{O C} R_{i}^{2} f\left(\alpha_{i}\right) \ln \left({ }^{R_{i} / R_{f_{i}}}\right)
\end{aligned}
$$

where $f(r)$ is defined by

$$
f(r)=\frac{1}{\sin ^{2} r}\left[1-\cos r \sqrt{1-\frac{11}{12} \sin ^{2} r}+\frac{1}{\sqrt{11.12}} \ln \frac{1+\frac{11}{12}}{\sqrt{\frac{11}{12}} \cos r+\sqrt{1-\frac{11}{12} \sin ^{2} r}}\right]
$$

The function $f(r)$ is tabulated in Table 8.1 of Ref. 36, for $7^{0}$ intervals for $0 \leq r \leq 90^{\circ}$. Table 8.1 of Ref. 36 is reproduced here as Table 1 . Please note that $f(r)$ is within 5\% error if replaced by 1 for $r$ up to $67^{\circ}$. 
Table 1 Relative Average Effective Strain $f(\alpha)$ and Shear Losses

\begin{tabular}{|c|c|c|c|c|c|c|c|c|}
\hline$\alpha^{0}$ & $f(\alpha)$ & $\frac{\alpha}{\sin ^{2} \alpha}-\cot \alpha$ & $\alpha^{0}$ & $f(\alpha)$ & $\frac{\alpha}{\sin ^{2} \alpha}-\cot \alpha$ & $\alpha^{\circ}$ & $f(\alpha)$ & $\frac{\alpha}{n^{2} \alpha}$ \\
\hline 0 & 1.00000 & 0 & & & & \\
\hline 1 & I. .00001 & 0.011636 & 31 & 1.00672 & 0.37539 & 61 & 1.03603 & 0.83746 \\
\hline 2 & 1.00003 & 0.023275 & 32 & 1.00721 & 0.38854 & 62 & 1.03784 & 0.85632 \\
\hline 3 & 1.00006 & 0.034920 & 33 & 1.00772 & 0.40180 & 63 & 1.03974 & $0.875+9$ \\
\hline 4 & 1.00010 & 0.046573 & 34 & 1.00825 & 0.41516 & 64 & 1.04174 & 0.89500 \\
\hline 5 & 1.00016 & 0.058237 & 35 & 1.00881 & 0.42864 & 65 & 1.04384 & 0.91 .184 \\
\hline 6 & 1.00023 & 0.069915 & 36 & 1.00939 & 0.44224 & 66 & 1.04605 & 0.93503 \\
\hline 7 & 1.00031 & 0.081611 & 37 & 1.01000 & 0.45596 & 67 & 1.04838 & 0.95559 \\
\hline 8 & 1.00041 & 0.093327 & 38 & 1. 01063 & 0.46981 & 68 & I. .05082 & 0.97653 \\
\hline 9 & 1.00052 & $0.1050 i$ & 39 & 1.01129 & 0.48380 & 69 & 1.05340 & $0.99 i 87$ \\
\hline 10 & 1.00064 & 0.11683 & 40 & 1.01198 & 0.49792 & 70 & 1.05613 & 1.01961 \\
\hline 11 & 1.00078 & 0.12862 & 41 & 1.01270 & 0.51218 & 71 & 1.05900 & $1.0+178$ \\
\hline 12 & 1.00093 & 0.14045 & 42 & 1.01345 & 0.52660 & 72 & 1.06204 & $1.06: t 38$ \\
\hline 13 & 1.00109 & 0.15231 & 43 & 1. 01423 & 0.51117 & 73 & 1. 06526 & $1.087+15$ \\
\hline 14 & 1.00127 & 0.16421 & 44 & 1.01505 & 0.55590 & 74 & $1.0686 \vec{i}$ & 1. 11099 \\
\hline 15 & 1.00146 & 0.17614 & 45 & 1.01590 & 0.57080 & 75 & 1.07228 & 1.13503 \\
\hline 16 & 1.00167 & 0.18813 & 46 & 1.01679 & 0.58587 & 76 & 1.07611 & 1.15958 \\
\hline 17 & 1.00189 & 0.20016 & 47 & 1.01772 & 0.60111 & 77 & 1.08018 & 1.18467 \\
\hline 18 & 1.00212 & $0.21: 23$ & 4.8 & 1.01869 & 0.61655 & 78 & 1.08451 & 1.21031 \\
\hline 19. & 1.00237 & $0.22 \div 37$ & 49 & 1.01970 & 0.63217 & 79 & 1.08912 & 1.23653 \\
\hline 20 & 1.00264 & 0.23656 & 50 & 1.02075 & 0.64800 & 80 & 1.09404 & 1.26335 \\
\hline 21 & 1.00292 & 0.24881 & 51 & 1.02185 & 0.66403 & 81 & 1.09928 & 1. 29080 \\
\hline 22 & 1.00322 & 0.26112 & 52 & 1.02300 & 0.68027 & 82 & 1.10 .488 & 1.31890 \\
\hline 23 & 1.00354 & 0.27350 & 53 & $1.02+20$ & 0.6967 .4 & 83 & 1.11087 & $1.3 \cdot 768$ \\
\hline 24 & 1.00387 & 0.28595 & 54 & $1.025+6$ & $0,713.1 .6$ & $8: 4$ & 1.11727 & 1.37717 \\
\hline 25 & 1.00422 & 0.298 .18 & 55 & 1.02677 & 0.73037 & 85 & $1.12+13$ & 1.40740 \\
\hline 26 & 1.00459 & 0.31108 & 56 & 1.02814 & 0.74755 & 86 & 1.131 .48 & $1.138 \div 0$ \\
\hline 27 & 1.00498 & $0.3237 i$ & 57 & 1.02958 & $0.76498^{\circ}$ & 87 & 1.13935 & 1.47020 \\
\hline 28 & 1.00538 & 0.33653 & 58 & 1.03108 & 0.78268 & 88 & $1.1 .4 \div 80$ & $1.5028+4$ \\
\hline 29 & 1.00581 & $0.3 \cdot 4939$ & 59 & 1.03265 & 0.80066 & 89 & $1.1568 \pi$ & 1.53636 \\
\hline 30 & 1.00625 & 0.3623. & 60 & $1.03 .+30$ & 0.81891 & 90 & 1.16660 & 1.57080 \\
\hline
\end{tabular}


(3) The Shear Power in the core: $\dot{w}_{S C}$

The shear power over surfaces of velocity discontinuity $\Gamma_{2 c}$ and

$\Gamma_{1 c}$, by Eq. (8.12) of Ref. 36 , becomes

$$
\left.\begin{array}{rl}
\dot{w}_{S C} & =\frac{(k+1) \sigma_{O C}}{\sqrt{3}} \int_{0}^{\alpha_{i}} v_{O C} \sin \theta\left(2 \pi r_{O i} \sin \theta r_{i} d \theta\right. \\
& =\frac{(k+1)}{\sqrt{3}} \pi \sigma_{O C} v_{O C} R_{i}^{2}\left(\frac{\alpha_{j}}{\sin ^{2} \alpha_{j}}-\cot \alpha_{i}\right)
\end{array}\right\}
$$

where

$$
\begin{array}{ll}
k=0 & \text { for core fracture } \\
k=1 & \text { for sleeve fracture }
\end{array}
$$

(4) The Friction Power along the Bearing of the Die: $W_{f}$

The friction power losses between the sleeve and the cylindrical portion of the die (bearing of the die) are independent of the assumed velocity field. They are

$$
\dot{w}_{f}=\frac{2}{\sqrt{3}} m \pi \sigma_{O S} V_{f x} R_{f}^{2}\left(\frac{L}{R_{f}}\right)
$$

The velocity $V_{\text {fx }}$ is the slaeve velocity $V_{f s}$ unless sleeve fracture is anticipated, in which case $V_{f x}=V_{f c}$.

\section{(5) The Internal Power of Deformation in the Sleeve: $W_{i s}$} The interial power of deformation in the sleeve $\dot{W}_{i s}$, by the toroidal
field, is obtained as follows:

$$
\dot{w}_{i s}=\frac{2}{\sqrt{3}} \sigma_{0 s} \int_{\alpha_{i}}^{\alpha} \int_{r_{\star}}^{r_{0 s}} \sqrt{\frac{1}{2} \dot{\varepsilon}_{i j} \dot{\varepsilon}_{i j}} 2 \pi(r \sin \theta+e) r d \theta d r(37)
$$

where $\frac{1}{2} \quad \dot{\varepsilon}_{i j} \dot{\varepsilon}_{i j}=\frac{1}{2}\left(\dot{\varepsilon}_{r r}^{2}+\dot{\varepsilon}_{\theta \theta}{ }^{2}+\dot{\varepsilon}_{\phi \phi}^{2}\right)+\dot{\varepsilon}_{r \theta}^{2}$

$\left[\dot{\varepsilon}_{r r}^{2}, \quad \dot{\varepsilon}_{\theta \theta}^{2}, \dot{\varepsilon}_{\phi \phi}^{2}\right.$ and $\dot{\varepsilon}_{r \theta}^{2}$ were calculated and listed in Eqs. (22).] 
$\alpha_{i}$. is defined by Eq. (17)

$r_{f s}^{*}$ is defined by Eq. (10) and

$r_{\text {os }}$ is defined by Eq. (6)

After linearization with respect to $e$ and $\varepsilon$, Eq. (31) becomes

$\dot{w}_{\text {is }}=2 \pi \sigma_{0 S} V_{\text {os }} R_{0}^{2}\left\{\left(\ln \frac{R_{0}}{R_{f}}\right) f(\alpha)-\left(\frac{R_{i}}{R_{0}}\right)^{2} \ln \left(\frac{R_{0}}{R_{f}}\right) f\left(\alpha_{i}\right)\right.$

$-\frac{e}{R_{0}}\left\langle\left(2 \ln \frac{R_{0}}{R_{f}}-\frac{R_{0}}{R_{f}}+1\right) f(\alpha)+\left(\frac{R_{0}}{R_{f}}-1-2 \frac{R_{0}}{R_{i}} \ln \frac{R_{0}}{R_{f}}\right)\left(\frac{R_{i}}{R_{0}}\right)^{2} f\left(\alpha_{i}\right)\right.$

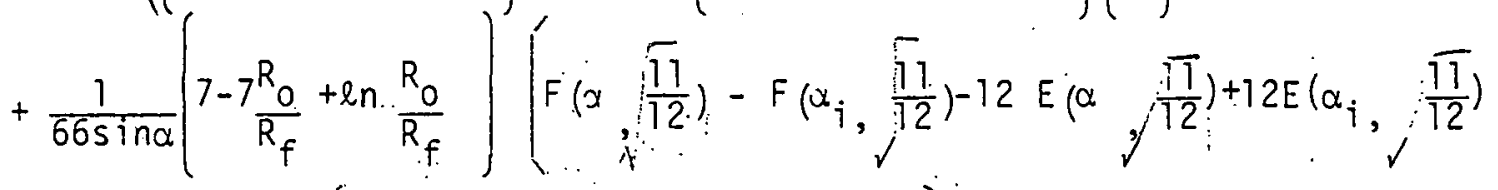

$-\frac{2}{\sin ^{2} \alpha} \ln \frac{R_{0}}{R_{f}}\left[E\left(\alpha, \sqrt{\frac{17}{12}}\right)-E\left(\alpha_{i, j} \sqrt{\frac{17}{12}}\right)\right.$

$+4 \pi \sigma_{0 S} V_{0} R_{0}^{2} \frac{e}{R_{0}}\left\{\frac{\left(1-\frac{R_{0}}{R_{f}}+\varepsilon\right)}{2 \sin ^{2} \alpha}\left(f(\alpha) \sin ^{2} \alpha-f\left(\alpha_{i}\right) \sin ^{2} \alpha_{i}\right]\right.$

$+\frac{\left(\frac{R_{0}}{R_{f}}-1\right)}{\sin \alpha}\left[E\left(^{\alpha,} / \frac{11}{12}\right)-E\left(\alpha_{i}, j \frac{\overline{11}}{j \frac{1}{12}}\right)\right]$

$\left.-\varepsilon\left[2 n \frac{\sin \alpha\left(\cos \alpha_{i}+\sqrt{1-\frac{11}{12} \sin ^{2} \alpha_{j}}\right)}{\sin \alpha_{j}\left(\cos \alpha+\sqrt{\left.1-\frac{11}{12} \sin ^{2} \alpha\right)}\right.}-\sqrt{\frac{11}{12}} \frac{\sqrt{1-\frac{11}{12}} \sin ^{2} \alpha-\sqrt{\frac{11}{12}} \cos \alpha}{\sqrt{1-\frac{11}{12} \sin ^{2} \alpha_{i}-\frac{\sqrt{11}}{12} \cos \alpha_{j}}}\right]\right\}$ 
where

$$
\begin{aligned}
& F\left(r, \sqrt{\frac{11}{12}}\right)=\int_{\theta=0}^{r} \frac{d \theta}{\sqrt{1-\frac{11}{12} \sin ^{2} \theta}} \\
& E\left(r, \sqrt{\frac{17}{12}}\right)=\int_{\theta=0}^{r} \sqrt{1-\frac{11}{12} \sin ^{2} \theta} d \theta
\end{aligned}
$$

The integrals $F\left(r, \sqrt{\frac{17}{12}}\right)$ and. $E\left(r, \sqrt{\frac{11}{12}}\right)$ are the first and second incomplete elliptic integrals. Values of these elliptic integrals are presented in Table 2.

\section{(6) The Shear Power on the Interface : Winterface $_{\text {infer }}$}

The shear loss over the conical interface between the core and the sleeve is due to the discontinuity in the tangential components of the assumed velocity fields. Because the normal component is zero, the equation for the shear loss is

$\dot{W}_{\text {interface }}=\int_{\Gamma_{c s}} m_{\eta} \Gamma\left|\dot{u}_{r s}-\dot{u}_{r c}\right| d s$

where $m_{1}$ is the coefficient describing the bonding condition, $0 \leq m_{1} \leq 1$,

$\Gamma_{C S}$ is the conical surface of separation,

and $I$ is the lower of the two rations,

$\frac{\sigma_{O S}}{\sqrt{3}}$ and $\frac{\sigma_{O C}}{\sqrt{3}}$

Consequently, algebraic complications are avoided if the equivalent equation

$\dot{W}_{\text {interface }}=m_{1}\left|\int_{\Gamma_{c s}} \Gamma \dot{U}_{r s} d s-\int_{\Gamma_{c s}} \Gamma \dot{U}_{r c} d s\right|$

is used where the integrals are computed separately with different variables. 
TABLE 2 First, $F(\alpha, k)=\int_{\theta=0}^{\alpha} \frac{d \theta}{\sqrt{1-k^{2} \sin ^{2} \theta}}$, and second, $E(\alpha, k)=\int_{\theta=0}^{\alpha} \sqrt{1-k^{2} \sin ^{2} \theta}$ d $\theta$ kind elliptic

\begin{tabular}{|c|c|c|c|c|c|c|c|}
\hline \multirow{2}{*}{$\alpha^{\circ}$} & $\begin{array}{l}\text { First } \\
\text { Kind } \\
\end{array}$ & \multicolumn{2}{|c|}{ Second Kind } & \multirow{2}{*}{$\alpha^{0}$} & $\begin{array}{l}\text { First } \\
\text { Kind }\end{array}$ & \multicolumn{2}{|c|}{ Second Kind } \\
\hline & \multicolumn{2}{|c|}{$k=\sqrt{11 / 12}$} & $k=\sqrt{3 / 4}$ & & \multicolumn{2}{|c|}{$k=\sqrt{11 / 12}$} & $k=\sqrt{3 / 4}$ \\
\hline 0 & 10 & 0 & 0 & & & & \\
\hline 1 & .01745 & .01745 & .01745 & 46 & .89455 & .72704 & .74195 \\
\hline 2 & .03491 & .03490 & .03490 & 47 & .91881 & .73960 & .75553 \\
\hline 3 & .05238 & .05234 & .05234 & 48 & .94345 & .75196 & .76896 \\
\hline 4 & .06987 & .06976 & .06977 & 49 & .96849 & .76413 & .78225 \\
\hline 5 & .08737 & .08717 & .08718 & 50 & .99395 & .77609 & .79538 \\
\hline 6 & .10490 & .10454 & .10458 & 51 & 1.01985 & .78785 & .80837 \\
\hline 7 & .12245 & .12189 & .12195 & 52 & 1.04621 & .79941 & .82120 \\
\hline 8 & .14005 & .13921 & .13929 & 53 & 1.07304 & .81076 & .83388 \\
\hline 9 & $: 15768$ & .15649 & .15660 & 54 & 1.10038 & .82191 & .84641 \\
\hline 10 & .17535 & .17372 & .17387 & 55 & 1.12824 & .83284 & .85879 \\
\hline 11 & .19308 & .19091 & .19110 & 56 & 1.15665 & .84356 & .87101 \\
\hline 12 & .21086 & .20804 & 20830 & 57 & 1.18564 & .85407 & .88308 \\
\hline 13 & .22870 & .22511 & .22544 & 58 & 1.21523 & .86437 & .89501 \\
\hline 14 & .24661 & .24213 & .24253 & 59 & 1.24545 & .87445 & .90678 \\
\hline 15 & .26458 & .25907 & .25957 & 60 & 1.27633 & .88431 & .91839 \\
\hline 16 & .28264 & .27594 & .27655 & 61 & 1.30790 & .89396 & .92986 \\
\hline 17 & .30078 & .29274 & .29347 & 62 & 1.34020 & . 90339 & .94119 \\
\hline 18 & .31900 & .30945 & .31032 & 63 & 1.37326 & .91261 & .95236 \\
\hline 19 & .33732 & .32608 & .32710 & 64 & 1.40711 & . 92161 & .96339 \\
\hline 20 & .35574 & .34262 & .34381 & 65 & 1.44180 & .93039 & .97428 \\
\hline$\overline{21}$ & .37427 & .35906 & .36044 & 66 & 1.47735 & .93896 & .98502 \\
\hline 22 & .39290 & .37540 & .37699 & 67 & 1.51382 & .94731 & .99563 \\
\hline 23 & .41166 & . 39164 & .39346 & 68 & 1.55124 & .95545 & $1: 00610$ \\
\hline 24 & .43055 & .40777 & .40984 & 69 & 1.58965 & .96338 & 1.01643 \\
\hline 25 & .44956 & .42379 & .42612 & 70 & 1.62910 & .97111 & 1.02664 \\
\hline$\overline{26}$ & .46872 & .43970 & .44732 & 71 & 1.66963 & .97862 & 1.03672 \\
\hline 27 & .48802 & .45548 & .45842 & 72 & 1.71128 & .98594 & 1.04668 \\
\hline 28 & .50748 & .47113 & .47441 & 73 & 1.75409 & .99305 & 1.05652 \\
\hline 29 & .52710 & . 48666 & .49031 & 74 & 1.79810 & .99997 & 1.06624 \\
\hline 30 & .54689 & .50205 & .50609 & 75 & 1.84335 & 1.00671 & 1.07586 \\
\hline 31 & .56686 & .51730 & .52177 & 76 & 1.88986 & 1.01326 & 1.08537 \\
\hline 32 & .58702 & .53241 & .53733 & 77 & 1.93767 & 1.01963 & 1.09478 \\
\hline 33 & .60738 & .54738 & .55278 & 78 & 1.98679 & 1.02583 & 1.10410 \\
\hline 34 & .62793 & .56220 & .56811 & 79 & 2.03722 & 1.03187 & 1.11334 \\
\hline 35 & .64871 & .57686 & .58332 & 80 & 2.08896 & 1.03776 & 1.12249 \\
\hline 36 & .66971 & .59137 & .59841 & 81 & 2.14199 & 1.04350 & 1.13156 \\
\hline 37 & .69094 & .60571 & .61337 & 82 & 2.19628 & 1.04912 & 1.14057 \\
\hline 38 & .71242 & .61990 & .62820 & 83 & 2.25176 & 1.05461 & 1.14952 \\
\hline 39 & .73416 & .63391 & .64290 & 84 & 2.30836 & 1.05999 & 1.15841 \\
\hline 40 & .75616 & .64775 & .65746 & 85 & 2.36597 & 1.06528 & 1.16726 \\
\hline 41 & .77845 & .66142 & .67189 & 86 & 2.42448 & 1.07048 & 1.17607 \\
\hline 42 & .80103 & .67491 & .68619 & 87 & 2.48373 & 1.07562 & 1.18484 \\
\hline 43 & .82391 & .68823 & .70034 & 88 & 2.54356 & 1.08072 & 1.19359 \\
\hline 44 & .84712 & .70135 & .71436 & 89 & 2.60378 & 1.08577 & 1.20233 \\
\hline 45 & .87066 & .71429 & .72823 & 90 & 2.66420 & 1.09082 & 1.21106 \\
\hline
\end{tabular}




$$
\begin{aligned}
& \text { In the first integral, } \\
& d s=2 \pi\left(r \sin \alpha_{i}+e\right) d r \\
& \dot{u}_{r_{s}}=-v_{0 s} \frac{r_{0}}{r}\left(\frac{r_{0} \sin \alpha_{i}+e}{r \sin \alpha_{i}+e}\right) \quad \cos \alpha_{i}
\end{aligned}
$$

and the limits of the integration are

$$
\frac{R_{f i}-e}{\sin \alpha_{i}} \leq r \leq \frac{R_{i}-e}{\sin \alpha_{i}}
$$

In the second integral,

$$
\begin{aligned}
& d s=2 \pi r \sin \alpha_{i} d r \\
& \dot{u}_{r c}=-v_{o c} \frac{R_{i}^{2} \cos \alpha_{i}}{r^{2} \sin ^{2} \alpha_{i}}
\end{aligned}
$$

and

$$
\frac{R_{f i}}{\sin \alpha_{i}} \leq r \leq \frac{R_{j}}{\sin \alpha_{i}}
$$

The result of this shear loss computation then becomes

$$
\begin{aligned}
w_{\text {interface }} & =\frac{2 \pi}{\sqrt{3}} m_{i} \cdot \min \left[\sigma_{o c}, \sigma_{o s}\right] \cdot v_{n} \\
& \cdot\left\{\left\{R_{i}\left(R_{i}-e\right) \cot \alpha_{i} \ln \frac{R_{i}-e}{R_{f i}-e}-R_{i}^{2} \cot \alpha_{i} \ln \frac{R_{i}}{R_{f i}}\right\}\right.
\end{aligned}
$$

where min $\left[\sigma_{O C}, \sigma_{O S}\right]$ is the smaller of $\sigma_{O C}$ and $\sigma_{O S}$.

(7) The Shear Power in the Sleeve : $W_{\text {SS }}$

The shear power on the surfaces of velocity discontinuity $\Gamma_{1 s}$ and $\Gamma_{2 s}$ is given by the following two equations:

$$
\begin{aligned}
\dot{W}_{\Gamma_{2 s}} & =\frac{\sigma_{0 S}}{\sqrt{ }} \int_{\Gamma_{2 s}} v_{0} \sin \theta d s \\
& =\frac{\sigma_{0 S}}{\sqrt{3}} \int_{\alpha_{i}}^{\alpha}\left(v_{0} \sin \theta\right) \cdot 2 \pi\left(r_{0} \sin \theta+e\right) r_{0} d \theta
\end{aligned}
$$




$$
\text { and } \begin{aligned}
\dot{W}_{\Gamma_{1 s}} & =\left.\frac{\sigma_{0 s}}{j^{3}}\right|_{\Gamma_{1 s}} v_{f s} \sin (\theta-\psi)-\left(-\left.\dot{u}_{r s}\right|_{r=r_{f s}^{*}} \sin \psi \mid d s\right. \\
= & \frac{\sigma_{o s}}{\sqrt{3}} \int_{\alpha_{i}}^{\alpha}\left|v_{f s} \sin (\theta-\psi)-v_{o s} \frac{r_{o s}\left(r_{o s} \sin \theta+e\right)}{r_{f s}^{*}\left(r_{f s}^{*} \sin \theta+e\right)} \cos \theta \sin \psi\right| \cdot 2 \pi\left(r_{f s}^{*} \sin \theta+e\right) r_{f s}^{*} d \theta
\end{aligned}
$$

where $\psi$ is the angle between the plane normal to the surface $\Gamma_{15}$ and the radial direction of the toroidal coordinate, i.e.,

$$
\psi=\tan ^{-1}\left\{\begin{array}{ll}
\frac{1}{r_{f s}^{*}} & : \frac{d \cdot r_{f s_{-}}^{*}}{d \theta}
\end{array}\right\}
$$

The total shear loss in the sleeve is therefore

$$
\dot{w}_{S S}=\dot{w}_{\Gamma_{2 s}}+(1-k) \ddot{w}_{\Gamma_{1 s}}
$$

where $k=0$ for core fracture

and $k=1$ for sleeve fracture

The linearized results from Eqs. (44) and (45) are Eqs. (48) and (49) respectively:

$$
\begin{aligned}
& \dot{W}_{\Gamma_{2 s}}=\frac{2 \pi}{\sqrt{3}} \sigma_{0 s} V_{0 s_{0} R_{0}^{2}}^{2}\left\{\frac{2 \alpha-\sin 2 \alpha}{4 \sin ^{2} \alpha}-\frac{2 \alpha_{i}-\sin 2 \alpha i}{4 \sin ^{2} \alpha}\right. \\
& -\frac{e}{R_{0}} \cdot\left(\frac{\alpha}{\sin ^{2} \alpha}-\frac{\alpha_{j}}{\sin ^{2} \alpha}-\frac{\cos \alpha_{i}}{\sin \alpha}-\left(1-\frac{\sin \alpha_{j}}{\sin \alpha}\right)\right]
\end{aligned}
$$

and 


$$
\left.\begin{array}{l}
\dot{W}_{\Gamma_{1 s}}=\frac{2 \pi}{\sqrt{3}} \sigma_{0 s} V_{f s} R_{f}^{2}\left\{\frac{2 \alpha-\sin 2 \alpha}{4 \sin 2 \alpha}-\frac{2 \alpha_{j}-\sin 2 \alpha_{i}}{4 \sin ^{2} \alpha}\right. \\
+\frac{e}{R_{0}}<\frac{R_{0}}{R_{f}} \frac{\left(\cos \alpha_{j}-\cos \alpha\right.}{\sin \alpha}-\frac{2}{\sin \alpha}\left(\frac{R_{0}}{R_{f}}-1\right) \ln \left(\frac{\tan \frac{\alpha}{2}}{\tan \frac{\alpha_{i}}{2}}\right)-\frac{2 \alpha-\sin 2 \alpha}{2 \sin ^{2} \alpha} \\
+\frac{2 \alpha_{i}-\sin 2 \alpha_{i}}{2 \sin ^{2} \alpha}-2 \varepsilon \mid \frac{2 \alpha-\sin 2 \alpha}{4 \sin ^{2} \alpha}-\frac{2 \alpha_{i}-\sin 2 \alpha_{i}}{4 \sin ^{2} \alpha}-2\left(\cot \alpha_{i}-\cot \alpha\right) \\
\left.+\left(\alpha-\alpha_{i}\right)\right\}
\end{array}\right\}
$$

(8) The Friction Power between the Sleeve and the Surface of the Die: $\dot{w}_{f s}$ This friction loss is calculated by

$$
\dot{w}_{f s}=\frac{\sigma_{o S}}{\sqrt{3}} m \int_{\left\{r_{f s}^{*} \theta=\alpha\right.}^{r_{o s}} \cdot\left|\dot{U}_{r s) \theta=\alpha}\right| 2 \pi(r \sin \alpha+e) d r
$$

The linearized result of this friction consumption is

$$
\dot{W}_{f s}=\frac{2 \pi}{\sqrt{3}} m \sigma_{o s} V_{0} R_{0}^{2} \cot \alpha\left[\ln \frac{R_{0}}{R_{f}}+\frac{e}{R_{0}}\left(\frac{R_{0}}{R_{f}}-1-\ln \frac{R_{0}}{R_{f}}\right)\right]
$$

\section{(9) The Fracture Energy Associated with Core Fracture or STeeve Fracture : Wracture}

As mentioned in the concept of fracture, if core fracture occurs, there must have been volume separation along $\Gamma_{j}$. That is, velocity discontinuity appears in the directions of both the plane normal and the tangent of the $\Gamma_{1 c}$ surface. The energy consumption associated with this event. is

$$
\begin{aligned}
& \dot{W}_{\text {core }}=\sigma_{O C} \int_{\Gamma_{1 C}} \cdot \frac{\left(\overline{\left(\Delta V_{T}\right)^{2}}\right.}{3}+\Delta V_{N} d s \\
& \text { where } \Delta V_{N}=v_{f s} \cos \theta-v_{o c}\left(\frac{r_{o i}}{r_{f i}}\right)^{2} \cos \theta \\
& \Delta V_{T}=V_{f s} \sin \theta \\
& d s=2 \pi \cdot\left(r_{f j} \sin \theta\right) r_{f i} d \theta
\end{aligned}
$$


Similarly, the fracture energy for sleeve fracture can be estimated by

$\dot{W}_{\substack{\text { sleeve } \\ \text { fracture }}}=\sigma_{0 s} \int_{\Gamma_{1 s}} \sqrt{\left(\frac{\Delta V_{T}}{3}\right)+\left(\Delta V_{N}\right)^{2}} d s$

where

$$
\begin{aligned}
\Delta V_{N} & =\left|v_{f c} \cos (\theta-\psi)-\left(-\dot{u}_{r s}\right)_{r=r_{f s}} \cos \psi\right| \\
\Delta V_{T} & =\left|v_{f c} \sin (\theta-\psi)-\left(-\dot{u}_{r s}\right)_{r=r_{f s}^{*}} \sin \psi\right| \\
d s & =2 \pi\left(r_{f s}^{*} \sin \theta+e\right) r_{f s}^{*} d \theta
\end{aligned}
$$

Therefore,

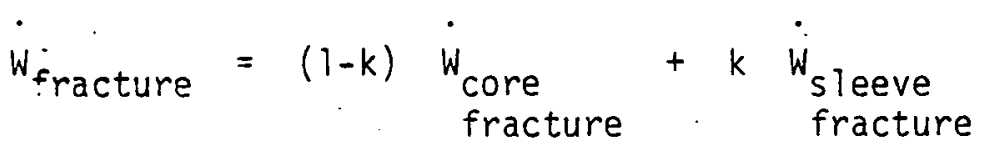

where

$$
\begin{aligned}
& k=0 \text { for core fracture } \\
& k=1 \text { for sleeve fracture }
\end{aligned}
$$

Linearized expressions for fracture energy are

$$
\dot{W}_{\text {core }}=\frac{\sigma_{\text {oc }}}{2 \sqrt{3}} \pi V_{f s} R_{f}^{2}\left(\frac{2 \alpha_{j}-\sin 2 \alpha_{i}}{\sin ^{2} \alpha_{i}}\right)\left(\frac{R_{j}}{R_{0}}\right)^{2}\left[1+2 \varepsilon \frac{e}{R_{0}}\left(\frac{R_{0}{ }^{2}}{R_{i}{ }^{2}}-1\right)\right]
$$
The linearized expression for $\dot{W}_{\text {sleeve fracture will be presented later. }}$
as Eq. $(62)$. 
Tile Characteristics of $\mathrm{J}^{*}$; The Contour Lines

The individual power terms of Eqs. (30), (44), (45), (50), (52) and (56) are presented in integral form. Precise analytical integration on these terms. was not performed. Before further analys is and approximations are made it is helpful to analyze the characteristics of each expression and of the total power $\mathrm{J}^{*}$. These characteristics are studied by performing numerical integration. In specific, the effect of the two pseudoindependent paramaters, namely, the eccentricity term (e) and the deviation term $(\varepsilon)$ on the individual power terms and on the total are of great interest. At first the effect of one paramater at a time is studied, and then their combined effect is presented in the form of contour lines.

When the arbitrary assumption is made that fracture does not occur and, therefore, $\varepsilon$ is assumed a priori to be zero, the power terms remain functions of eccentricity alone (e). For these conditions, for a typical bi-metal rod, typical characteristcs of the total power $\mathrm{J}^{*}$ are described in Fig. 13. Please note that a minimum power value is observed at $e=7.877 \times 10^{-3}$. Furthermore, it should be noted that the difference between $(i)$ the value of $j^{*}$ at $e=0$ and $(i j)$ the optimal value of $j^{*}$ is very small indeed. The evaluation by numerical methods must be made very carefully. All the characteristics described in this section are based on a preliminary exploratory program, and on few calculations, as designed to conserve budget. More in-depth rigorous programming and longer runs with higher precision factors are planned.

Observing that the specific value of $e=7.877 \times 10^{-3}$ provided a lowest value for $J^{*}$ at $\varepsilon=0$, it is then of interest to find the characteristics of $\mathrm{J}^{*}$ as a function of $\varepsilon$ when $e=7.877 \times 10^{-3}$ for positive values of $\varepsilon$. It should be noted from the section describing combined flow and fracture that positive values of $\varepsilon$ lead to the excessive deformalion of the sleeve and to core fracture.

The characteristics of $\mathrm{J}^{*}=J^{*}(\varepsilon)$ at $e=7.877 \times 10^{-3}$ for the specific bi-metal drawing are described in Fig. 14. Please note that this characteristic curve starts increasing as $\varepsilon$ increases from zero, reaches a peak, which is only incrementally above the value at $\varepsilon=0$, and drops down.

Since fracture has to initiate from the value of $\varepsilon=0$, the characteristics of Fig. 14 suggest that fracture initiation will require more power than that required for proportional flow, and thus proportional flow will prevail as the stable flow. The small values of $e$ and $\varepsilon$, and the small differences in total power associated with proportional flow or fracture, indicate that the stability of the stable flow is rather fragile. Any disturbance or initial defect in the material may shift the flow from proportional to fracture flow. Furthermore, the accuracy of the analys is depends on several assumptions, and the invalidity of any of these assumptions may trigger a change 
to failure mode. Some of these assumptions are homogeneity of each constituent, isotropy, perfectly plastic behavior, etc.

A failure mode is associated with a characteristic of $\mathrm{J}^{\star}$ where at the optimal value of eccentricity $\left(e=e_{o p t}\right)$, the slope of $J$ * as a function of $\varepsilon$ is negative, namely,

$$
\left.\frac{\partial J^{\star}}{\partial \varepsilon}\right|_{\begin{array}{l}
e=e_{o p t} \\
\varepsilon=0
\end{array}}<0
$$

The characteristics of Eq. (63) are described in Fig. 15.

In Figs. 16 and $17, J^{*}$ is plotted as a function of both $e$ and $\varepsilon$ in the vicinity of $e=\varepsilon=0$, for positive values of $e$ and $\varepsilon$. The contour lines are lines of equal $j^{*}$ values. Fig. 16 describes the contour lines for conditions that imply that no core fracture is expected. Note that the minimum value of $J^{*}$ occurs at $e>0$ but $\varepsilon=0$. For the case described by Fig. 17, the minimum occurs at $e>0$ and $\varepsilon>\dot{0}$ which means that core fracture is expected.

An important observation must be made here regarding the derivative of $\mathrm{J}^{\star}$ with respect to $\varepsilon$ at $e=0$. The original equations for the velocity field, the equations for power and the numerical solution for the characteristics of $\mathrm{J}^{\star}$ reveal that

$$
\frac{\partial J^{*}}{\partial \varepsilon}-\left.\right|_{e=\varepsilon=0} 0
$$

Thus, for core fracture to occur, the minimum point in Figs. 16 and 17 must occur at positive values of $e$ and $\varepsilon$.

\section{Criteria for Core Fracture}

Observation of Figs. 16 and 17 suggests that the following are necessary and sufficient conditions for core fracture to exist:

and

$$
\left.\begin{array}{l}
\left.\frac{\partial J^{\star}}{\partial e}\right|_{e=\varepsilon=0}<0 \\
\left.\frac{\partial J^{\star}}{\partial \varepsilon}\right|_{\begin{array}{l}
e>0 \\
\varepsilon=0
\end{array}} ^{<}
\end{array}\right\}
$$


Thus, the slope ${ }_{\star}$ of $J^{\star}$ at the origin, with respect to e, must * be negative so that $J$ for some positive valve of $e$ is lower than $J$ * at the origin. If this first condition is maintained, fracture may occur, but only if the following second necessary condition prevails. The second necessary condition requires that the minimum exists for a positive nonzero value of $\varepsilon$ (Fig. 17).

Numerical determination of these two conditions may lead to the construction of criteria for core fracture as presented in Figs. $18(a-d)$.

Figure 18(a) shows the criterion for core fracture in the domain of $\frac{\sigma_{O S}}{\sigma_{O C}}$ vs. ${ }_{\sigma}{ }^{R} R_{0}$ for differenent die angles $\left(10^{\circ}, 30^{\circ}, 50^{\circ}\right.$, and $\left.80^{\circ}\right)$ where $\frac{\sigma_{O S}}{\sigma_{O C}}$ represents the ratio of the yield strength of the sleeve to that of ${ }^{\sigma} O C_{\text {the core, and }} R_{i / R_{0}}$ is the radius ratio of the core to that of the sleeve before the deformation. In this criterion, the precent reduction $r \%$, friction coefficient $m$, bonding condition $m_{l}$ and relative back tension $\sigma_{x b}$ are preset to be $30 \%, 0.05,1.0$ and 0.0 respectively. If the selected combination of relative strength $\frac{\sigma_{O S}}{\sigma_{O C}}$ and size $\frac{R_{i}}{R_{O}}$ of the core and sleeve, for the above mentioned values of $r \%, m, m p$ and $\sigma_{x b} / \sigma_{0 S}$ falls under the curve of the specific die angle used, core fracture is expected. Otherwise, it is prevented.

Figures 18 (b) through 18 (d) are criteria for core fracture in the same domain as that of Fig. 18 (a). But, instead of changing die angle, the percent reduction, the friction coefficient, or the bonding condition are chosen to be variable for the curves in Fig. 18 (b) to 18 (d) respectively. In all the diagrams, the area under the curve predicts the fracture domain for those specific combinations of process parameters.

With the aid of these graphical criteria, the core fracture in codrawing can be easily prevented without going through traditional trialand-error procedures. Of course, the precision of the determination of specific process parameters, such as friction coefficient, bonding condition, and strength ratio of the materials, is reflected in the accuracy of the prediction.

Because of stability problems as described next, the further away the working conditions are from any particular criterion curve, the safer is the use of that criterion.

Figures 18 are also constructed from a set of equations derived by applying Eq. (65) to the power terms of Eq. (25). Figs. 16 and 17 reveal that the minimum of $J$ occurs with very small values of $e$ and $a$. Therefore, the values of the strain rate components in Eq. (22) have been 
linearized with respect to $\varepsilon$ and $e$ together with all other expressions. The analytical integration for the individual power terms became possible.

The linearized power terms are verified one by one against the numerically integrated power terms. Since the differentiation by Eq. (25) is called for at the origin, the differentiation for the determination of the necessary and sufficient conditions for core fracture is a precise solution.

Each step of the analytical integration and then of the differentiation is presently checked both analytically (once more) and against the numerical evaluation. This checking is a necessary and lengthy process and we defer presentation of the final equations subject to verification. Typing of the lengthy results, only after the check is made, will save both expense and perhaps embarassment (if corrections are called for).

\section{PROPERTIES OF THE SUPERCONDUCTING WIRE}

This phase of research was started in the month of August. The objectives of the program are to design, on a laboratory scale, a new forming process for manufacturing $\mathrm{Nb}_{3} \mathrm{Sn}$ wires, and to characterize and evaluate the microstructures of these products in an attempt to improve their superconducting performance.

In the first phase of this investigation, the bronze technique for production of $\mathrm{Nb}_{s} \mathrm{Sn}$ wires was reexamined and modified. The conventional wire drawing is to be replaced by the process of hydrostatic extrusion as ciescribed in Sec. 1. The use of the new process will improve not only the efficiency of the forming operation, but also the physical properties of the superconducting products.

It was decided that two sets of $\mathrm{Nb}$-bronze composite rods would be tested. One set nf rods with multifilaments of niobium in a bronze matrix will be supplied by the Airco Company; the other set with mullurilaments will be prepared by the Brookhaven National Laboratory (the Nb-bronze ratios will be the same as those used by the investigators at NBL). In addition, single crystals of $\mathrm{Nb}$ will also be used as the core material in the monofilamentary wire series. It is expected that the texture of the Nb core developed by the forming process will have an effect on the diffusion of $\mathrm{Sn}$ and, subsequently, on the microstructure of the $\mathrm{Nb}_{s} \mathrm{Sn}$ compound.

The composite rods will then be hydrostatically extruded, and annealed to form the superconducting $\mathrm{Nb}_{\mathrm{S}} \mathrm{Sn}$ compound following the bronze technique. The parameters to be investigated will be the percentage of deformation, the annealing time and temperature, and the Nb-bronze ratio. All these speci- 
mens will be carefully examined using optical and electron microscopy. Microhardness measurements will also be made to detect the inhomogeneity of the products. This program is expected to be completed in the summer of 1980 .

The second phase of this investigation will be focussed on the measurements of the superconducting parameters, $T_{C}$ and $J_{C}$ at the liquid helium temperature and under tensile loading. This phase of research will be carried out at the NBL with the guidance of Dr. Thomas Luhman, and is scheduled to be started in the summer of 1980 .

A second line of investigation will be designed to verify the theoretical findings (Sec. 2) by experimental observation. A set of monofilamentary rods of $\mathrm{Nb}$ and $\mathrm{Cu}-\mathrm{Sn}$ composites of different $\mathrm{Nb} / \mathrm{Cu}-\mathrm{Sn}$ deformed rods will be sectioned into halves and examined under microscope for fracture sites. The experimental results should verify the theoretical predictions investigated in phase two of the program. This program will be started in the month of December.

All the programs described above are in their early stages of development. The results and conclusions will be reported at a later date. 


\section{REFERENCES}

1 Walker, M. S., et al., "Properties and Performance of Fine-Filament Bronze-Process $\mathrm{Nb}_{3} \mathrm{Sn}$ Conductors," IEEE Transactions on Magnetics, Vol. Mag-15, No. 1, Jan. 1979. Published by the IEE Magnetic Soc., pp. 80-82.

2 Avitzur, B., "Metal-Forming Processes," John Wiley, 1980.

3 Avitzur, B., "Recent Developments in Wire Making,". To be published in the new edition of the Wire Handbook by the Wire Association and in Proceedings, National Symposium on Large Deformation, New Delhi, India, Dec. 17-19, 1979.

4 Robertson, J., "Method of and Apparatus for Forming Metal Articles," British Patent No. 19,356, 14 October 1893;

U. S. Patent No. 524,504, 14 August 1894.

5 Bridgman, P. W., Physics of High Pressure, International Textbooks of Exact Sciences, G. Bell \& Sons, Ltd., London, 1949.

6 Pugh, H. L1. D. and Gunn, D. A., "The Cold Extrusion of Brittle Materials against a.Hydrostatic Pressure," NEL Report 31 .

7 Pugh, H. L1. D., and Ashcroft, K., "The Hydrostatic, or Ramless, Extrusion of Metals by Fluid Pressure," NEL Report 32.

8 Pugh, H. Lì. D., and Green, U., "The Behavior of Metals under High Hydrostatic Pressure," Part II. Tensile and Torsion Tests, NERL Plasticity Report 128, October, 1956.

9 Pugh, H. LT. D., and Low, A. H., "The Hydrostatic Extrusion of Difficult Metals.," J. Inst. Meta1s, pp. 201-217. March, 1965.

10 Fiorentino, R. T., Sabroff, A. M., and Boulger, F. W., "Advances in Hydrostatic Extrusion," Tool Manufacturing Engr., pp.77-83, August, 1963.

11 Green, D., "An Experimental High Speed Machine for Practical Exploitation of Hydrostatic Extrusion," J. Inst. Metals, Vol. 93, Pp. 65-70, 1964-1965.

12 Fuchs, F. J., Jr., "Production Metal Forming with Hydrostatic Pressures," ASME Paper 65-PROD-17, June; 1965. 
13 Beresnev, B. I., Vereshchagin, L. F., and Ryabinin, Yu. N., "Characteristics of the Rheological Behavior of Metals Excruded under Hydrostatic Pressure" (in Russian), Izv. Akad, Nauk SSSR, Otdl. Tech. Nauk, 1957 , (5), pp. 48-55.

14 Beresnev, B. I., Vereshchagin, L. F., and Ryabinin, Yu. N., "Extrusion of Metals by Means of Liquid under High Pressure" (in Russian), Izv. Akad, Nauk, SSSR, Nekh, i Mashin., 1959, 7 (i), pp. 128-132.

15 Beresnev, B. I., Vereshchagin, L. F., and Ryabinin, Yu. N., "Conditions of Extrusion and Variations of Mechanical Properties of Metals in Their Extrusion by a High Pressure Fluid" (in Russian), Inzh.-fz zh., 1960, 3 (12), pp. 43-48.

16 "1600/80 Ton Hydraulic Extrusion Press," The Engineer, Sept. 8, 1967, pp. 312-314.

17 Kobe Hydrostatic Extrusion Press. Catalog No. 35141 , Kobe-Steel, Ltd. Machinery Division, 1 chome, WakinohamaCho, Fukiai-ku, Kobe, Japan.

18 Hayashi, M., Jokota, M., Kordo, T., and Hirata, M., "A Study on the Hydrostatic Extrusion of Fine Wire," Sumitomo Electric Industries, Ltd., Osaka, Japan, 1974, to be published.

19 Bobrowsky, A., Stack, E. A., and Austen, A., "Extrusion and Drawing Using High Pressure Hydraulics," Am. Soc. Tool Manufacturing Eng., Paper SP65-33, 1964.

20 Avitzur, B., "Hydrostatic Extrusion," J. of Eng. for Ind., Trans. ASME Series B., Vol. 87, Nov. 1965, pp. 487-494.

21. Avitzur, B., and Sortais, H. C., "Experimental Study of Hydrostatic Extrusion," J. of Basic Eng., Trans. ASME, Series D, Vol. 88, No. 3, Sept. 1966, pp. 658-668.

22 Hillier, M. J., A Hydrodynamic Model of Hydrostatic Extrusion, Intern. J. Production Res., Vol. 5, p. 171, 1967.

23 Garner, J. N., and Rice, W. B., "Predictions of Conditions of Central Bursting in Hydrostatic Extrusion," Annals of the CIRP, Vol. 25/1/1976, pp. 165-167.

24 Pugh, H. L1. D., "Recent Developments in Cold Forming, "Bulleid Memorial Lecture, 1965, Vols. IIIA and IIIB, The University of Nottingham Press, Nottingham, England, 1965. 
25 Fuchs, F. J., "Hydrostatic Wire Extrusion," The Wire Journal, Oct. 1970, pp. 105-113.

26 Jelinek, F. J., and Carmichael, D. C., "Experimental Evaluation of Hydrostatic Extrusion for the Fabrication of Multifilament Superconducting Wire," Battelle Report to Division of Magnetic Fusion Energy, Dept. of Energy, Project No. G5080-6301(774), 1976.

27 Alexander, J. M., and Lengyel, B., British Pat. Applications $12,326 / 65,56,241 / 66,41,249 / 67$.

28 Alexander, J. M., and Lengye 1, B., Proc. Inst., Mech. Eng., 1966,180 (31) 317.

29 Lengyel, B., and Alexander, J. M., High Pressure Eng. Conf., Proc. Inst. of Mech. Eng., London, 1968, 182 (3c), 207.

30 Lengye1, B., "A Semi-Continuous Hydrostatic Extrusion Process," Metals \& Materials, Jan. 1968, pp. 9-15.

31 McAllan, J. W., British Pat. Application No. 39,796, 1965.

32 Slater, H. K., and Green D., "Augmented Hydrostatic Extrusion of Continuous Bar," High Pressure Eng. Conf., London, 11-15 Sept. 1967, Inst. Mech. Eng., London, 1967-68, 182 (3c), Paper No. 14, pp. 109-114.

33 Austen, A. R., "Method and Apparatus for Extrusion," United States Pat. No. 3,999,415 dated Dec. 28, 1976. Also, United States Pat. No. 4,028,919 dated June 14, 1977.

34 Fuchs, F. J., and Schmeh1, G. L., "Continuous Hydrostatic Extrusion," presented and published, NEL/AIRAPT International Conference on Hydrostatic Extrusion, University of Stering, Scotland, June $13 \cdots 15,1973$.

35 Sabroff, A. M., and Fiorentino, R. J., "High Reduction Orawing," U. S. Patent No. 3,328,998, July 1967.

36 Avitzur, B., "Metal Forming : Processes and Analysis," McGraw-Hi11, 1968, 500 pp.; Krieger, 1979.

37 Hayashi, M., Jokota, M., Kondo, T., and Hinata, M., "A Study on the Hydrostatic Extrusion of Fine Wire," Sumitomo Electric Industries, Ltd., Osaka, Japan, 1974, to be published.

38 Avitzur, B., "Analysis of Center Bursting Defects in Drawing and Extrusion," Journal of Engineering for Industry, Trans. ASME Series B., Vol. 90, No. 1, Feb., 1968, pp. 79-91. 
39 Avitzur, B., "Metal Forming : The Application of Limit Analysis", Marcel Dekker Inc., New York, 1980.

40 Rouse, H., (ed.), "Advanced Mechanics of Fluids," John Wiley \& Sons, Inc., New York, 1959.

41 Sokolnikoff, I. S., Mathematical Theory of Elasticity, 2nd ed., McGraw-Hil1, New York, 1956, pp. 177-184. 


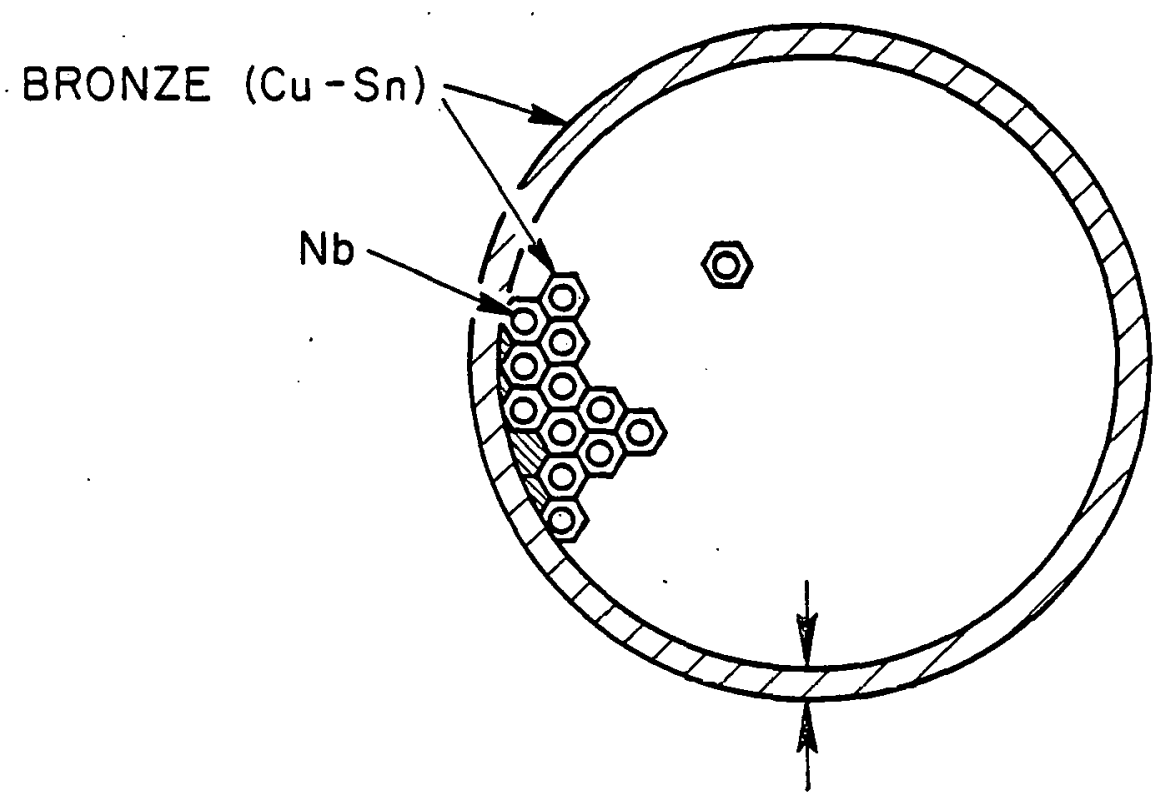

(a) BILLET ASSEMBLY.

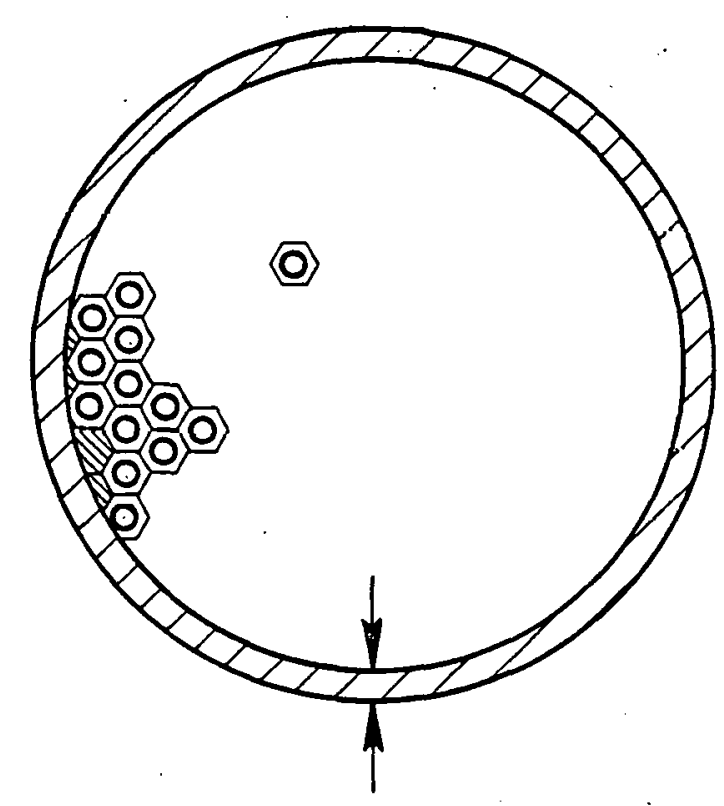

(b) AFTER EXTRUSION.

FIG.1 BILLET ASSEMBLY-TYPICAL $\mathrm{Nb}_{3}$ Sn SUPERCONDUCTING WIRE. 


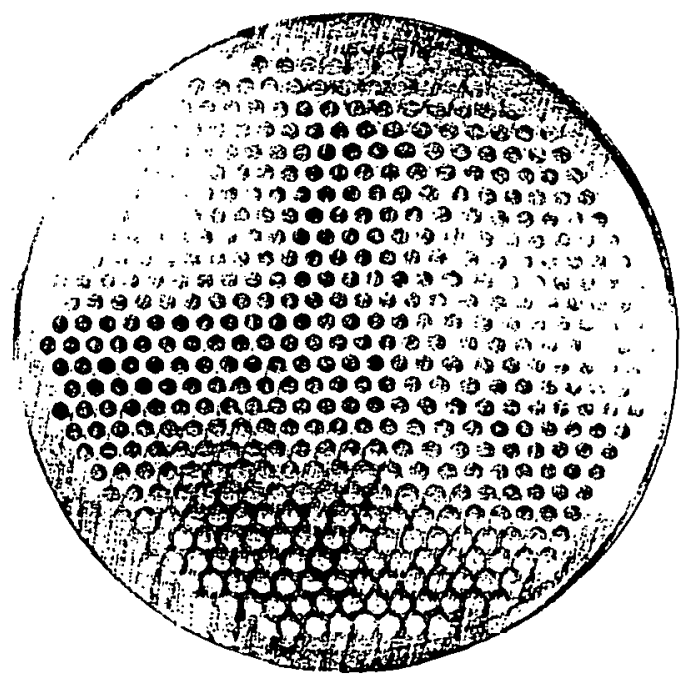

(c) POLISHED SPECIMEN OF FULL BILLET.

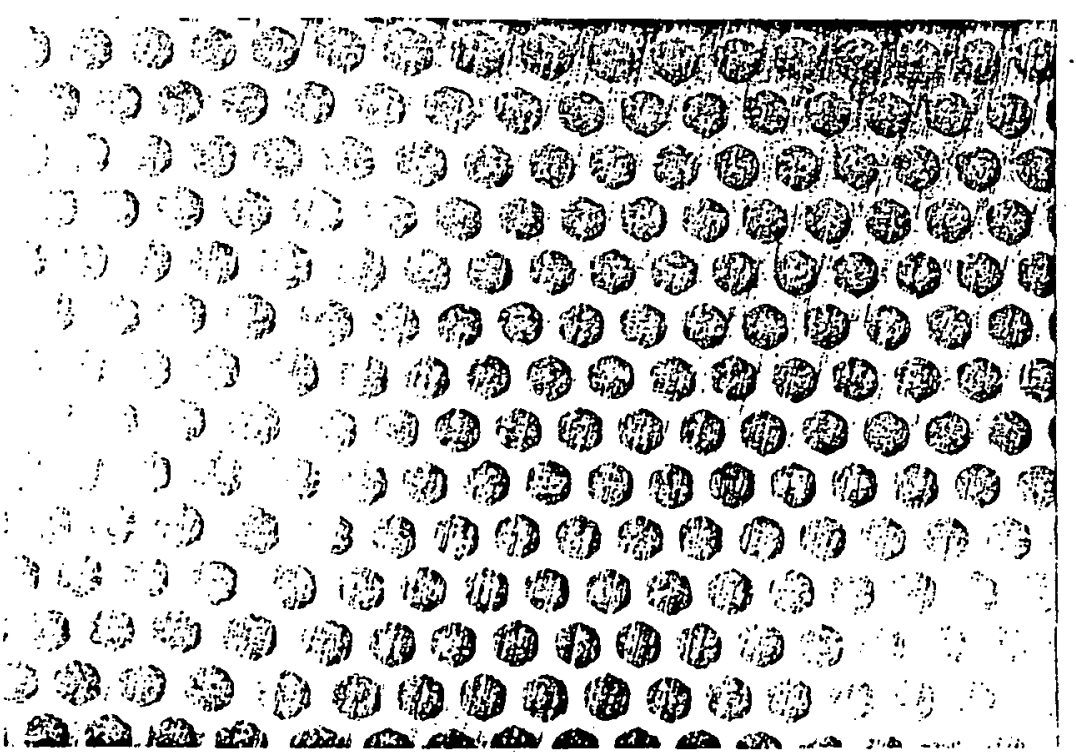
(d) ENLARGED PICTURE OF POLISHED SECTION.

\section{FIG.1 BILLET ASSEMBLY - TYPICAL $\mathrm{Nb}_{3} \mathrm{Sn}$ SUPER - CONDUCTING WIRE.}




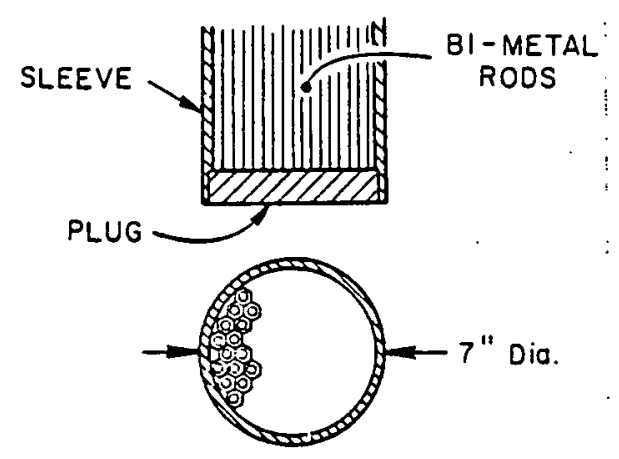

(1) BILLET ASSEMBLY

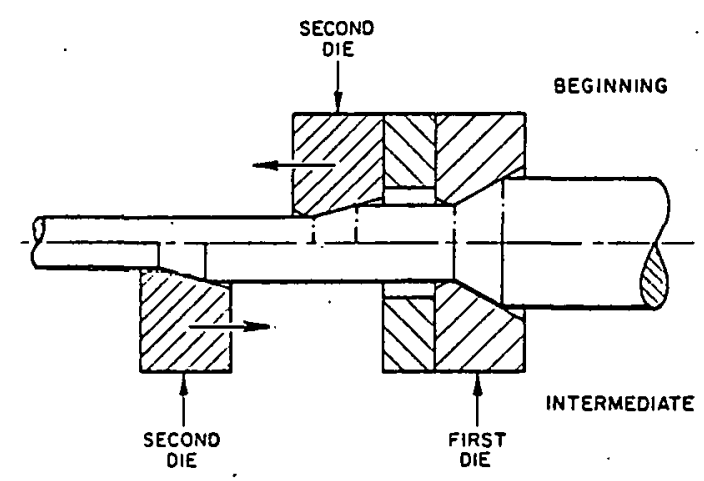

(2) MULTISTAGE HYDROSTATIC EX TRUSION OR SEMI-CONTINUOUS HYD. EXT. OR BY THE CON-DIE PROCESS.
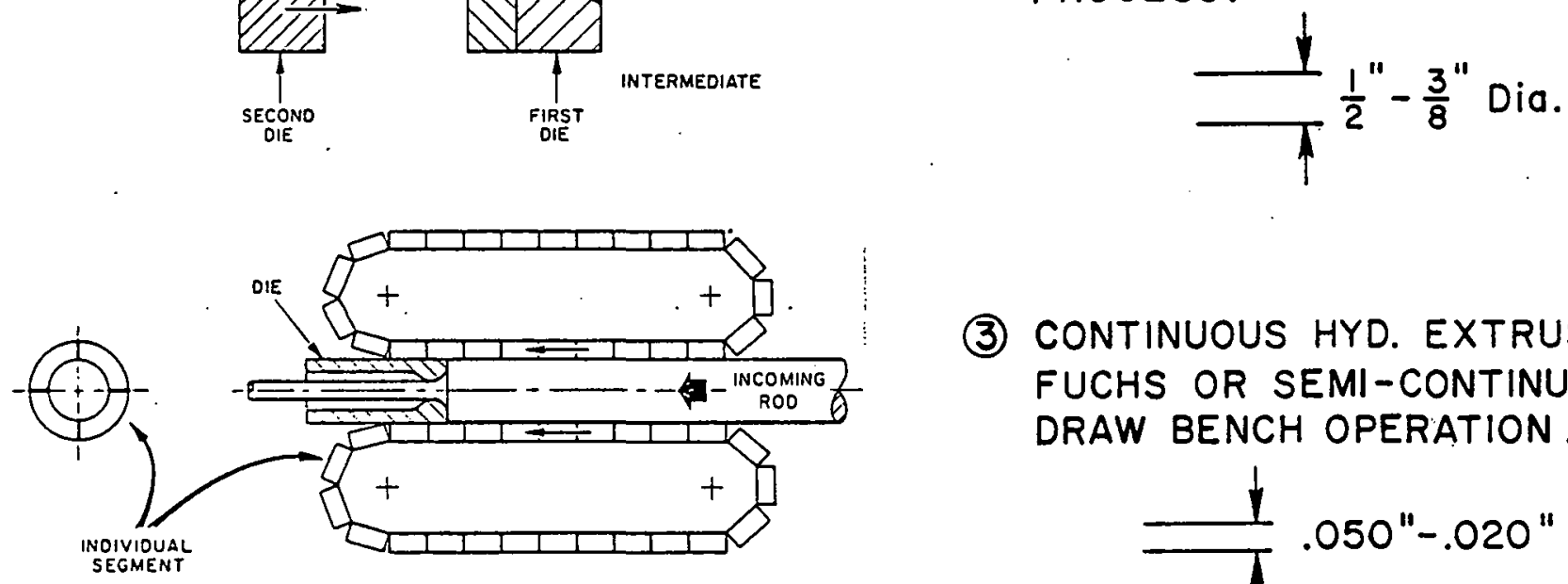

(3) CONTINUOUS HYD. EXTRUSION BY FUCHS OR SEMI-CONTINUOUS DRAW BENCH OPERATION .
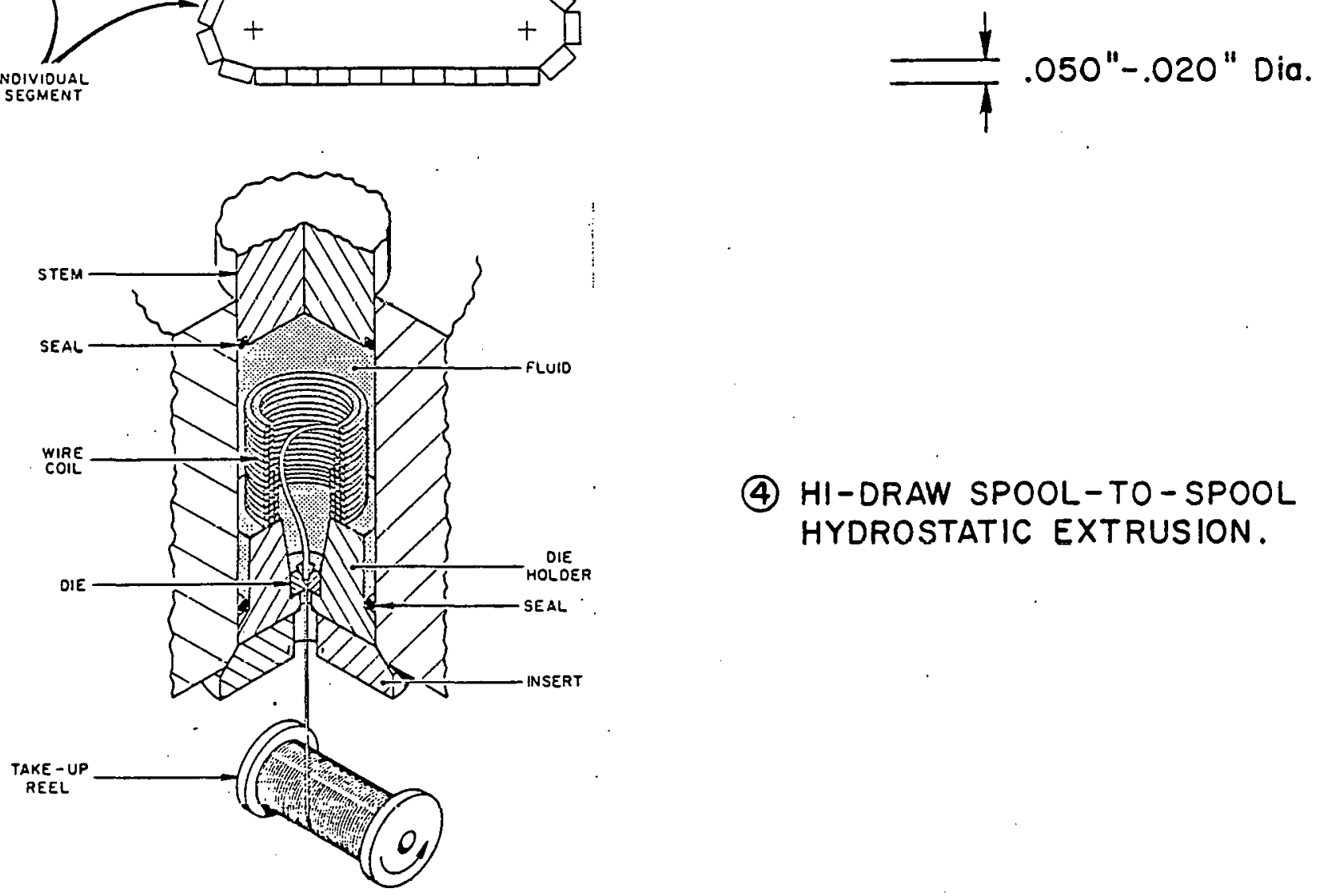

(4) HI-DRAW SPOOL-TO-SPOOL HYDROSTATIC EXTRUSION.

FIG.2 HYPOTHETICAL PRODUCTION LINE. 


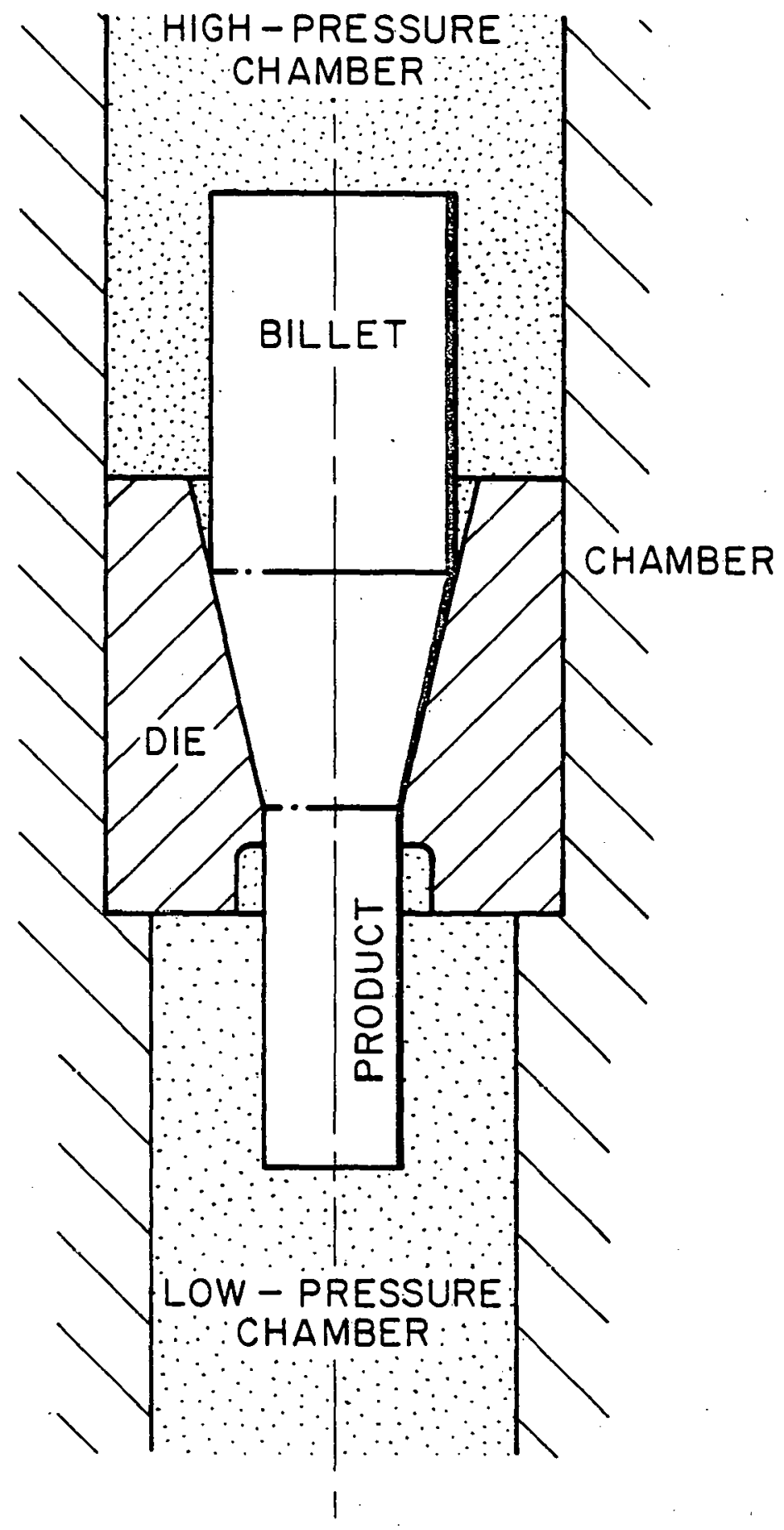

FIG.3 PRESSURE - TO - PRESSURE EXTRUSION. 


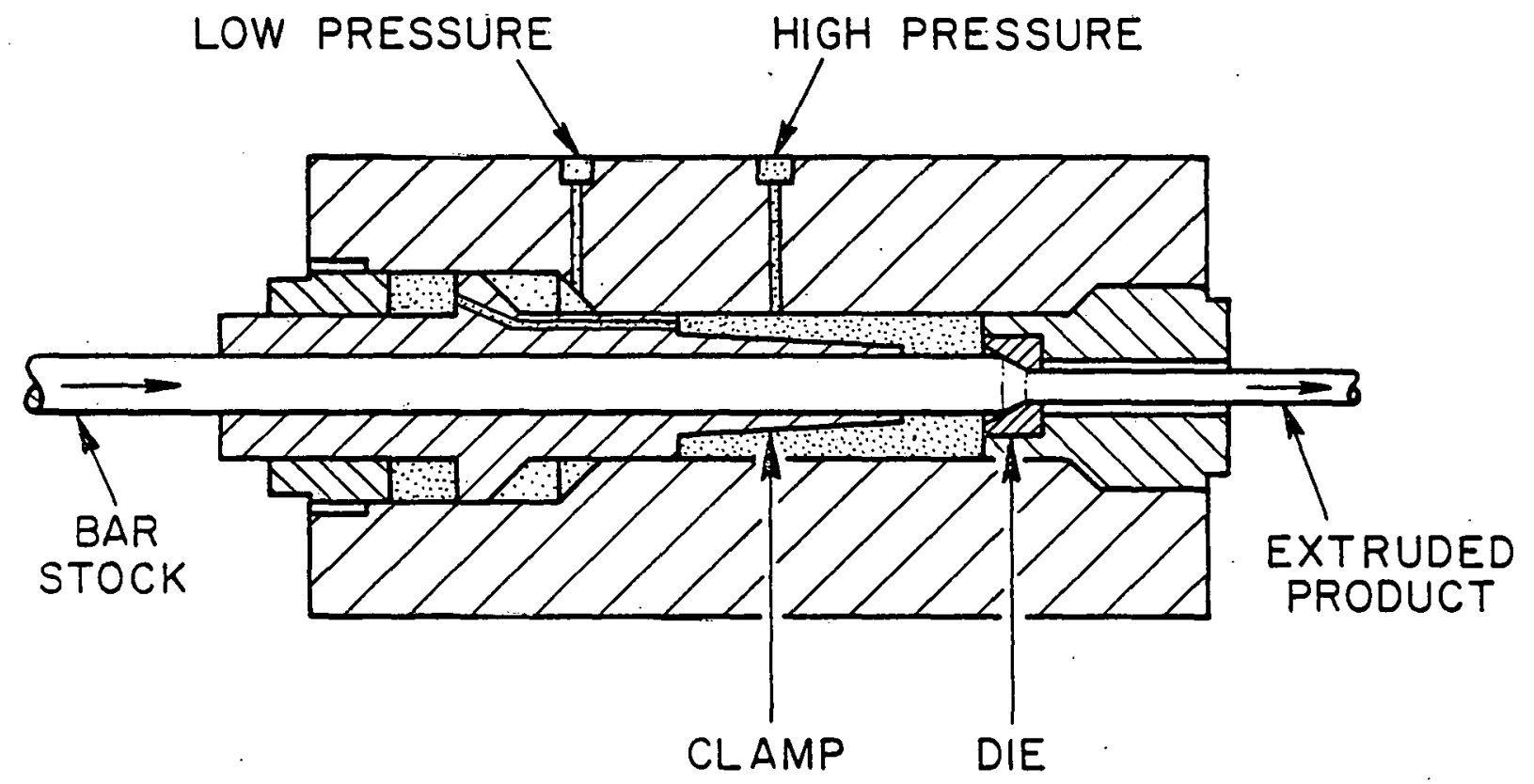

FIG.4 SEMI-CONTINUOUS HYDROSTATIC EXTRUSION. (BY GREEN) 


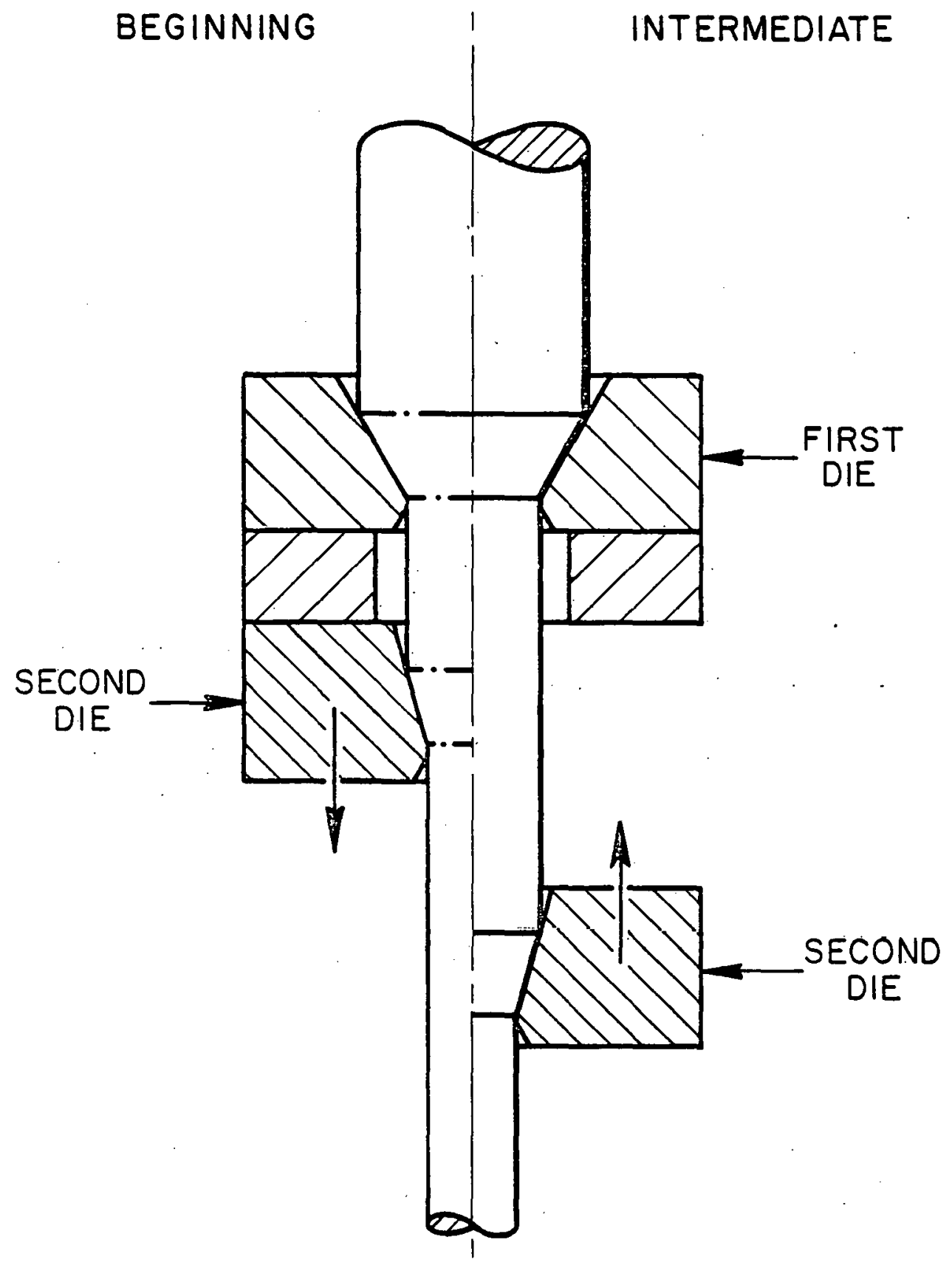

FIG.5 MULTISTAGE SEMICONTINUOUS EXTRUSION BY AUSTEN. 


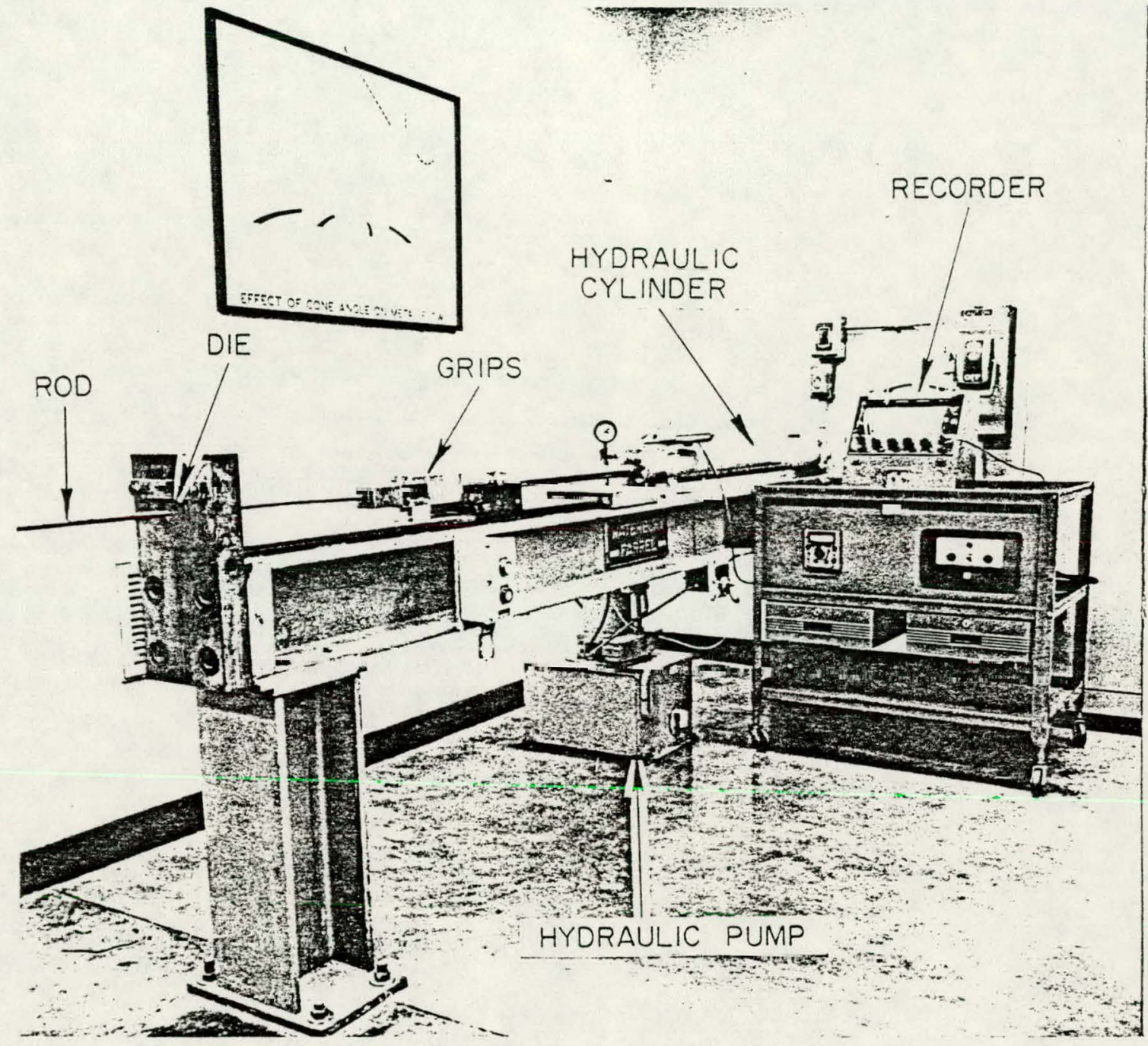

FIG.6 DRAW BENCH. 


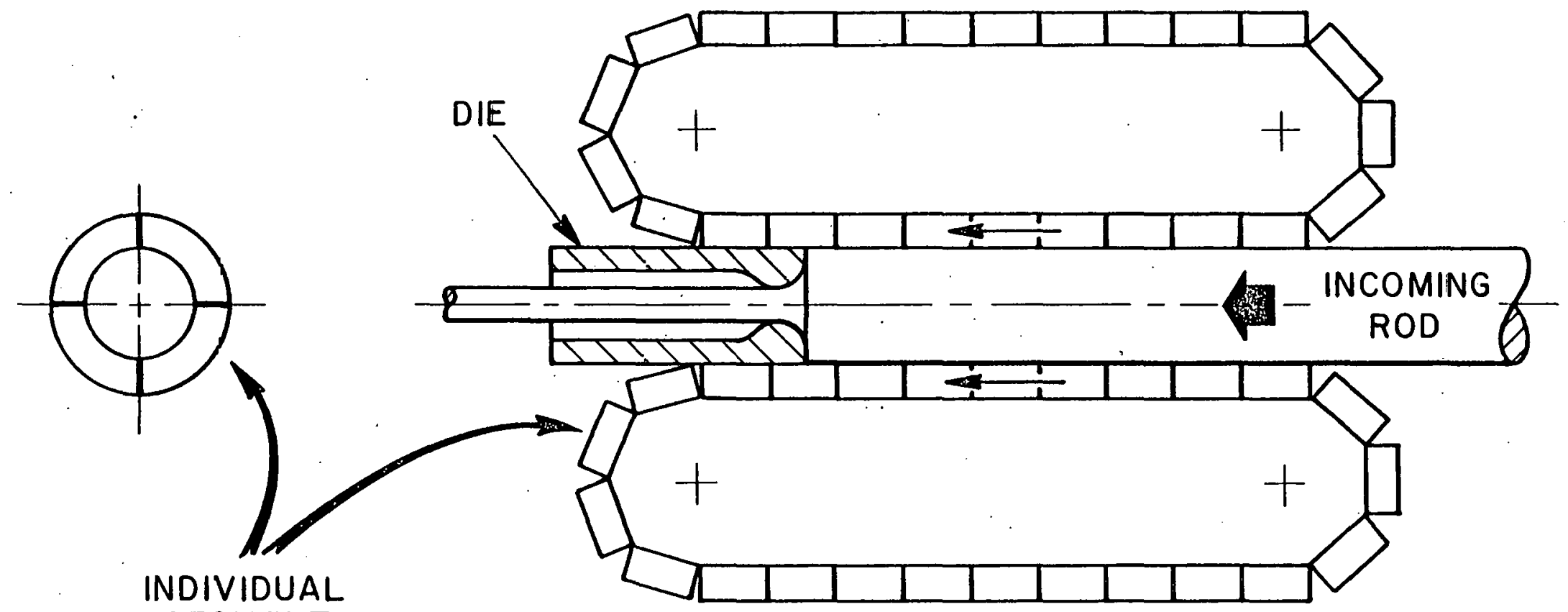

FIG.7 CONTINUOUS CHAMBER. 


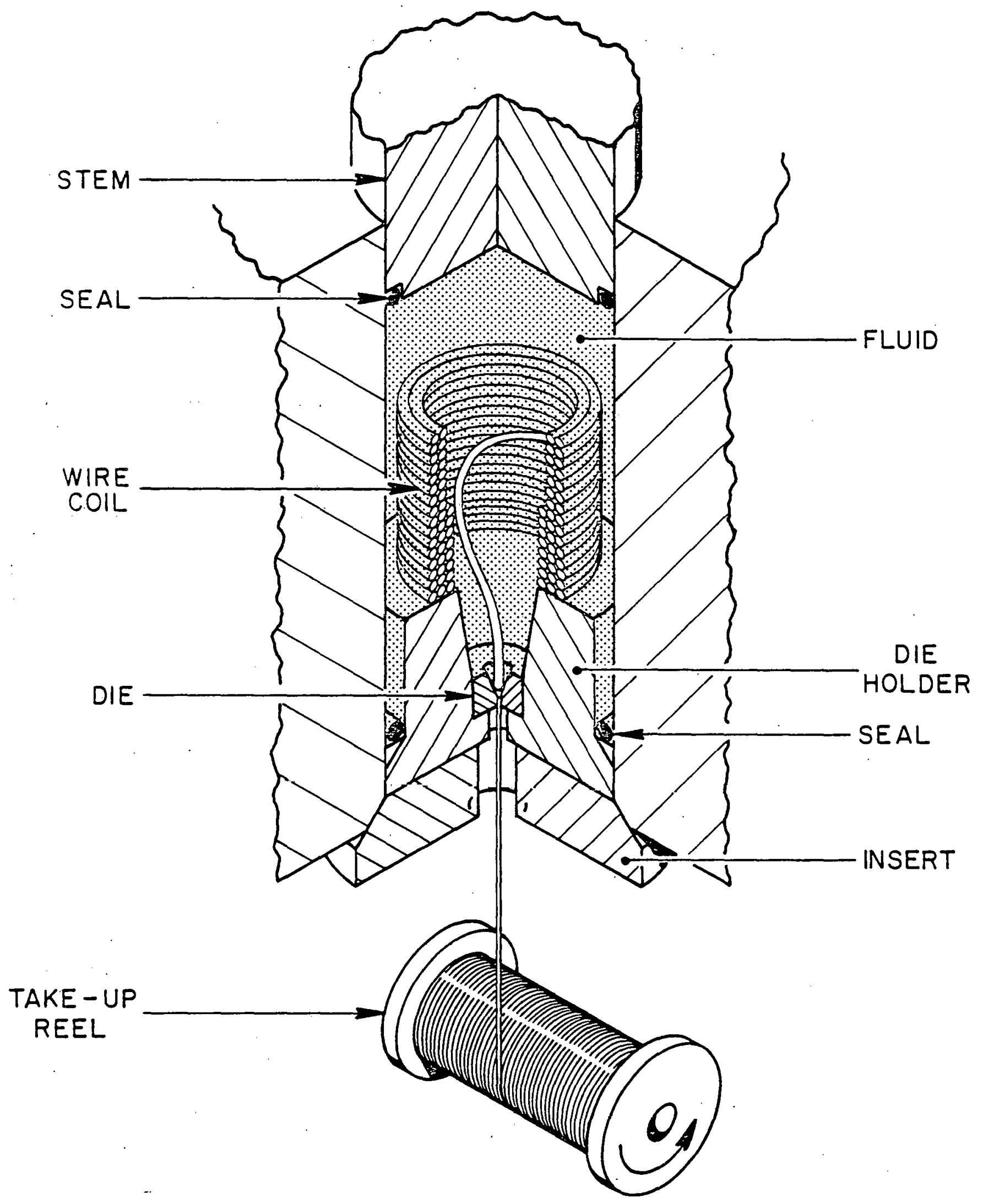

FIG.8 WIRE SPOOL. 


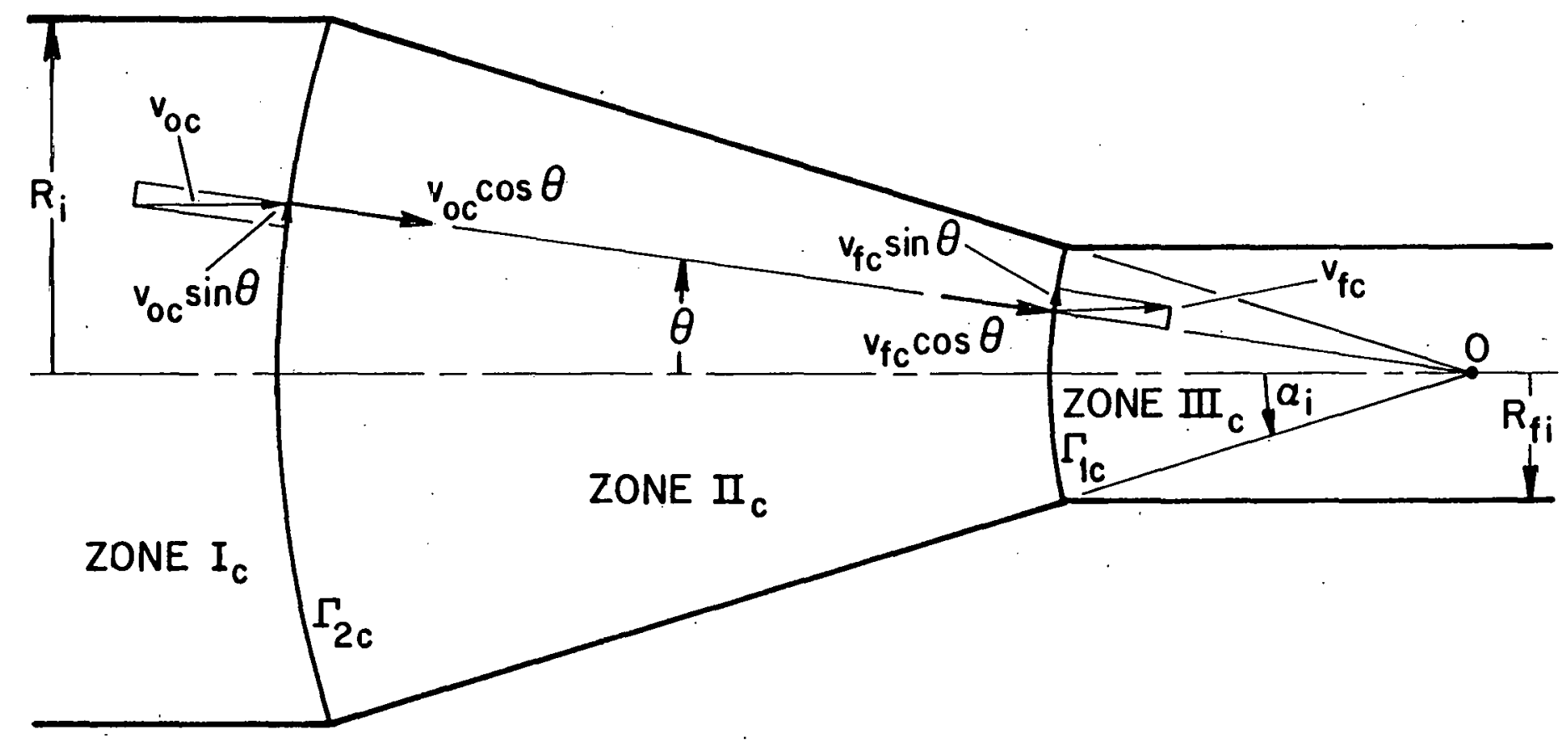

FIG.9 THE SPHERICAL VELOCITY FIELD FOQR THE CORE. 


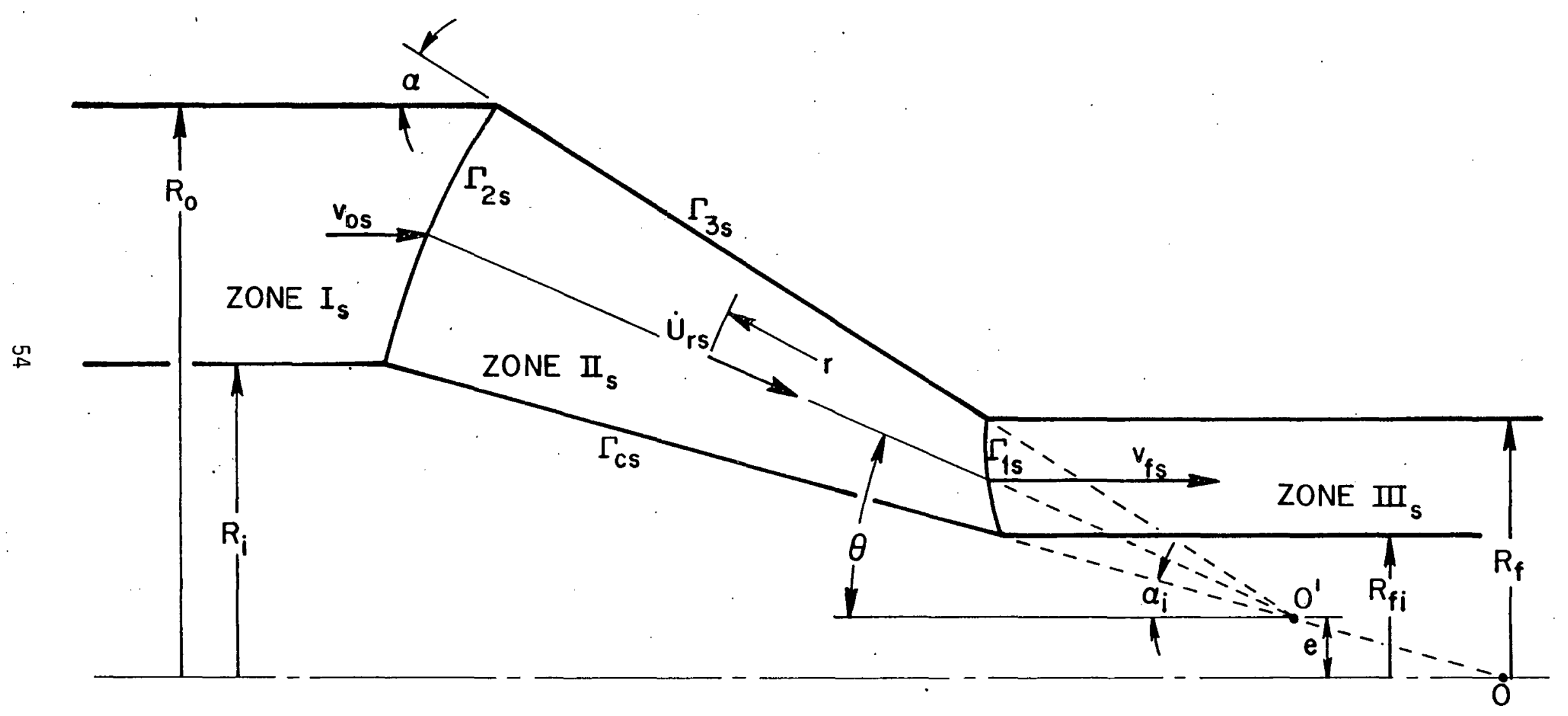

FIG.IO THE TOROIDAL VELOCITY FIELD FOR THE SLEEVE. 


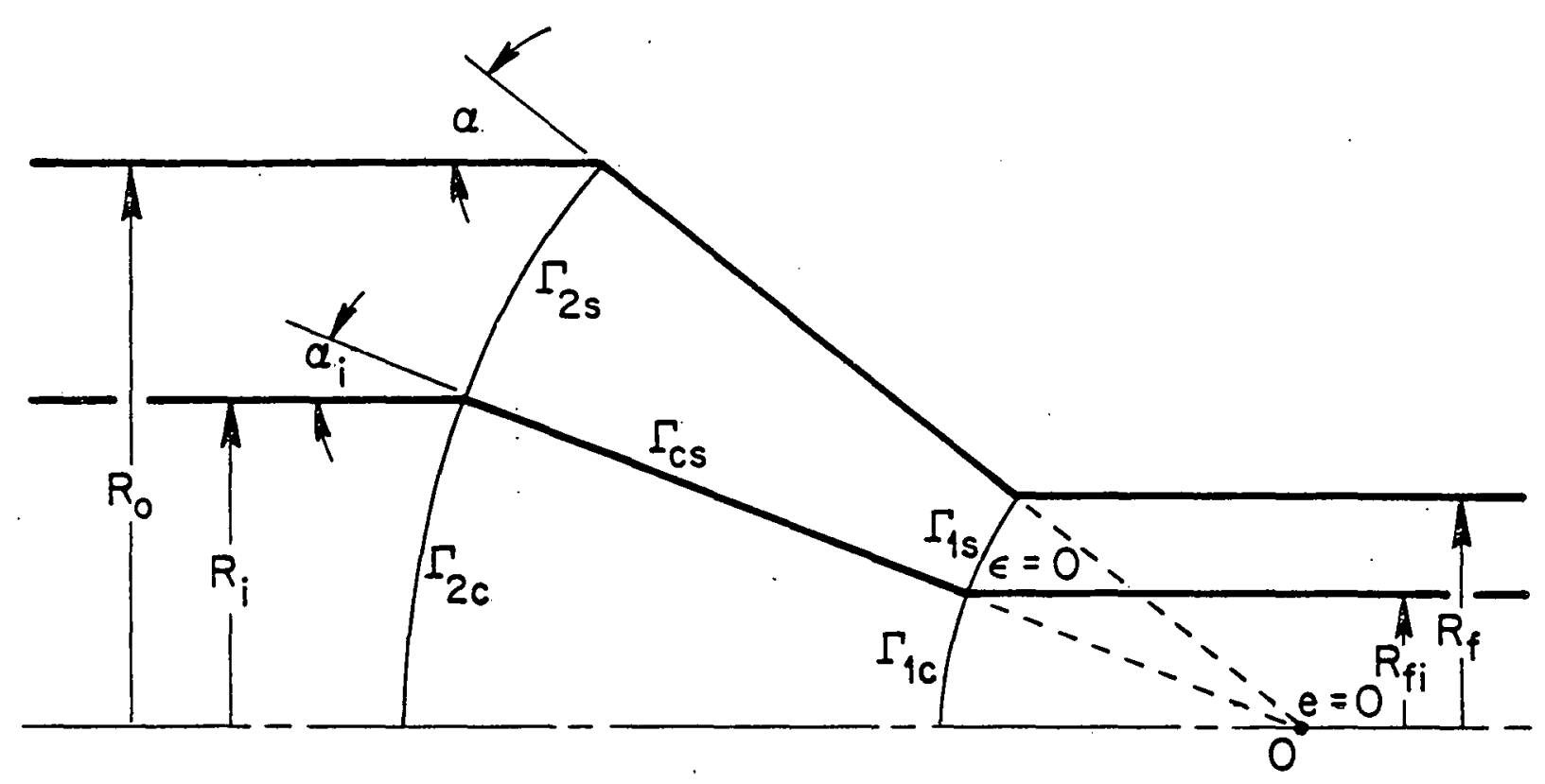

(a) PROPORTIONAL FLOW OF THE SLEEVE WITH $e=0$.

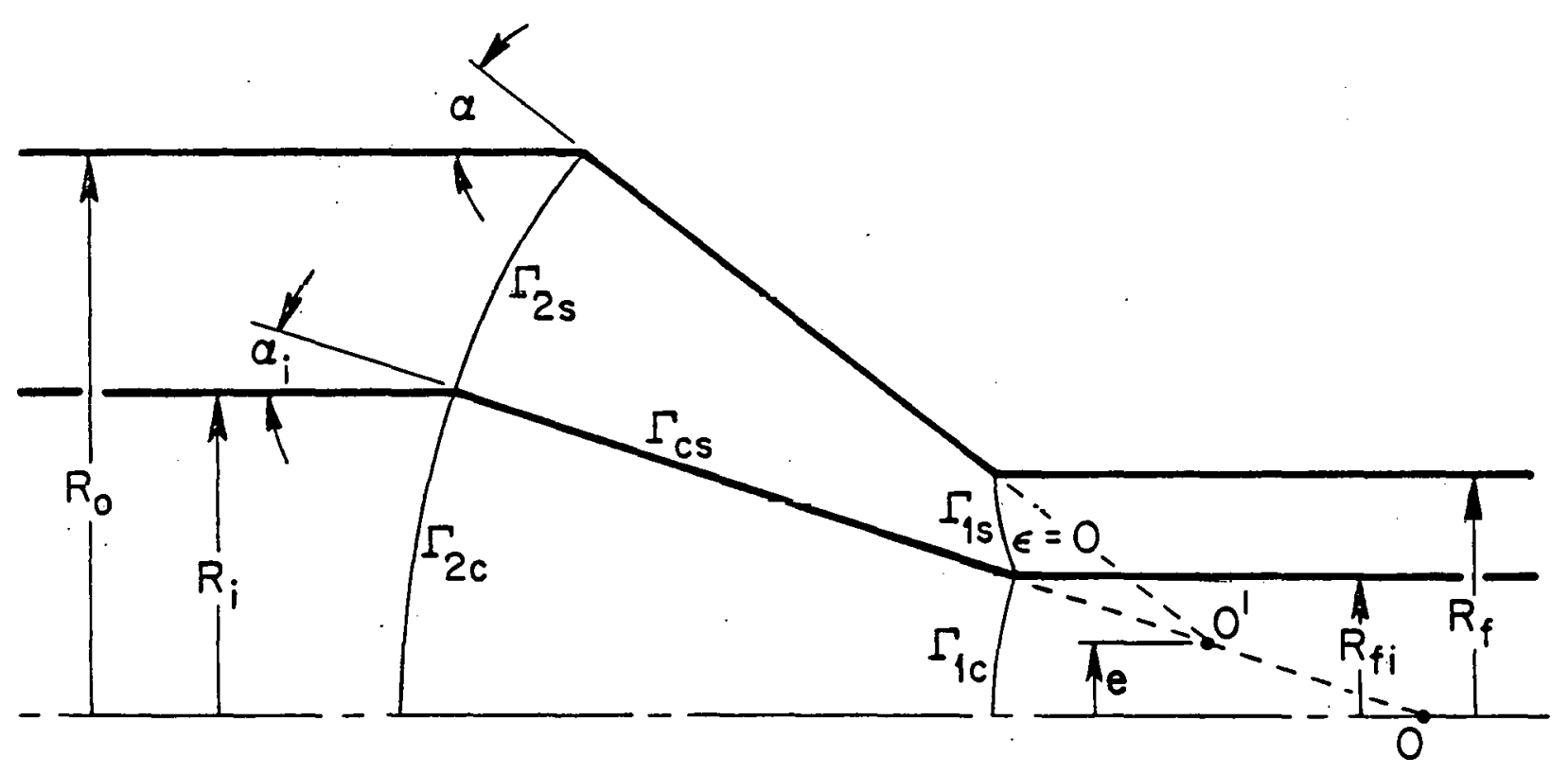

(b) PROPORTIONAL FLOW OF THE SLEEVE WITH $\mathrm{e} \neq 0$.

FIG.II DETAIL VARIATIONS IN TOROIDAL VELOCITY FIELD. 


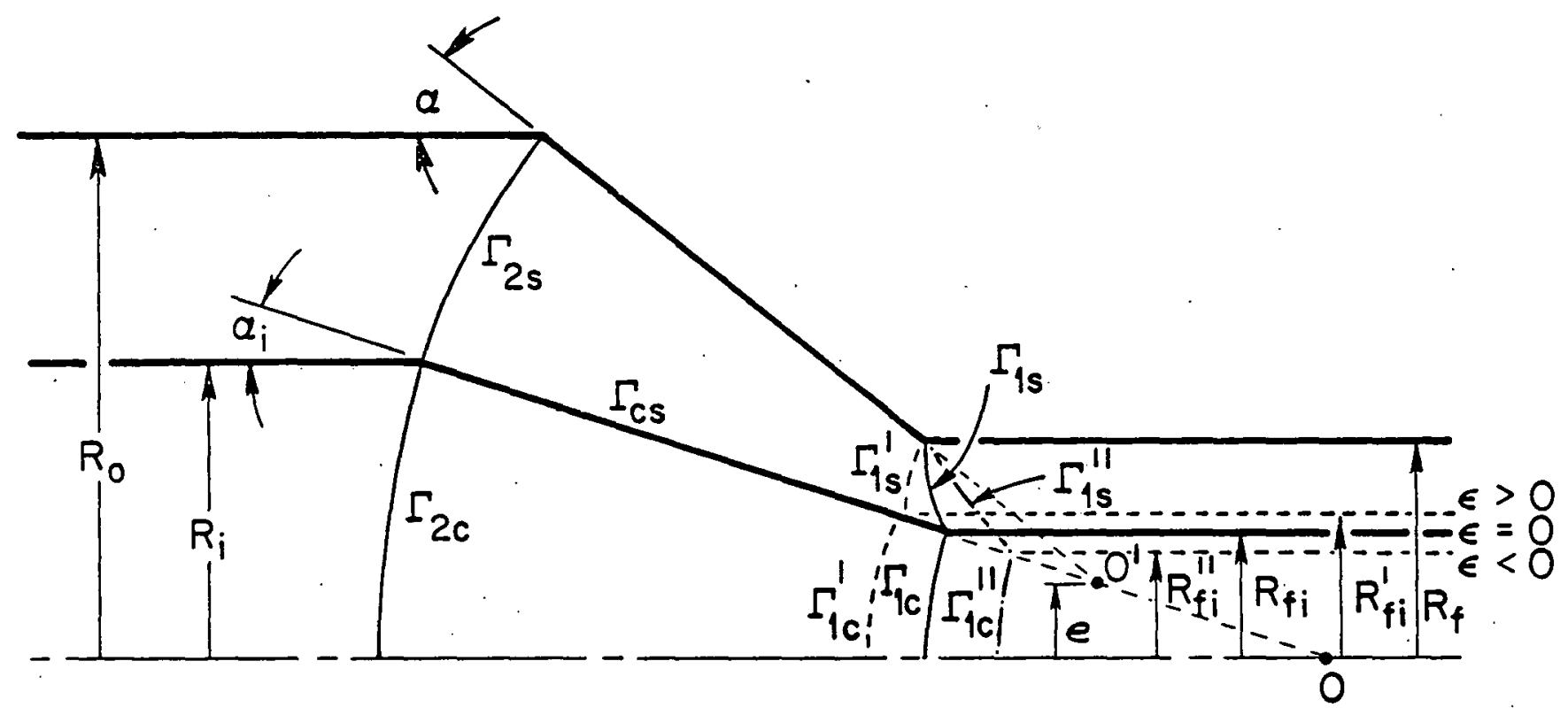

(c) THE EFFECT OF $\epsilon$ ON SLEEVE THINNING AND THICKENING.

FIG.II DETAIL VARIATIONS IN TOROIDAL VELOCITY FIELD. 


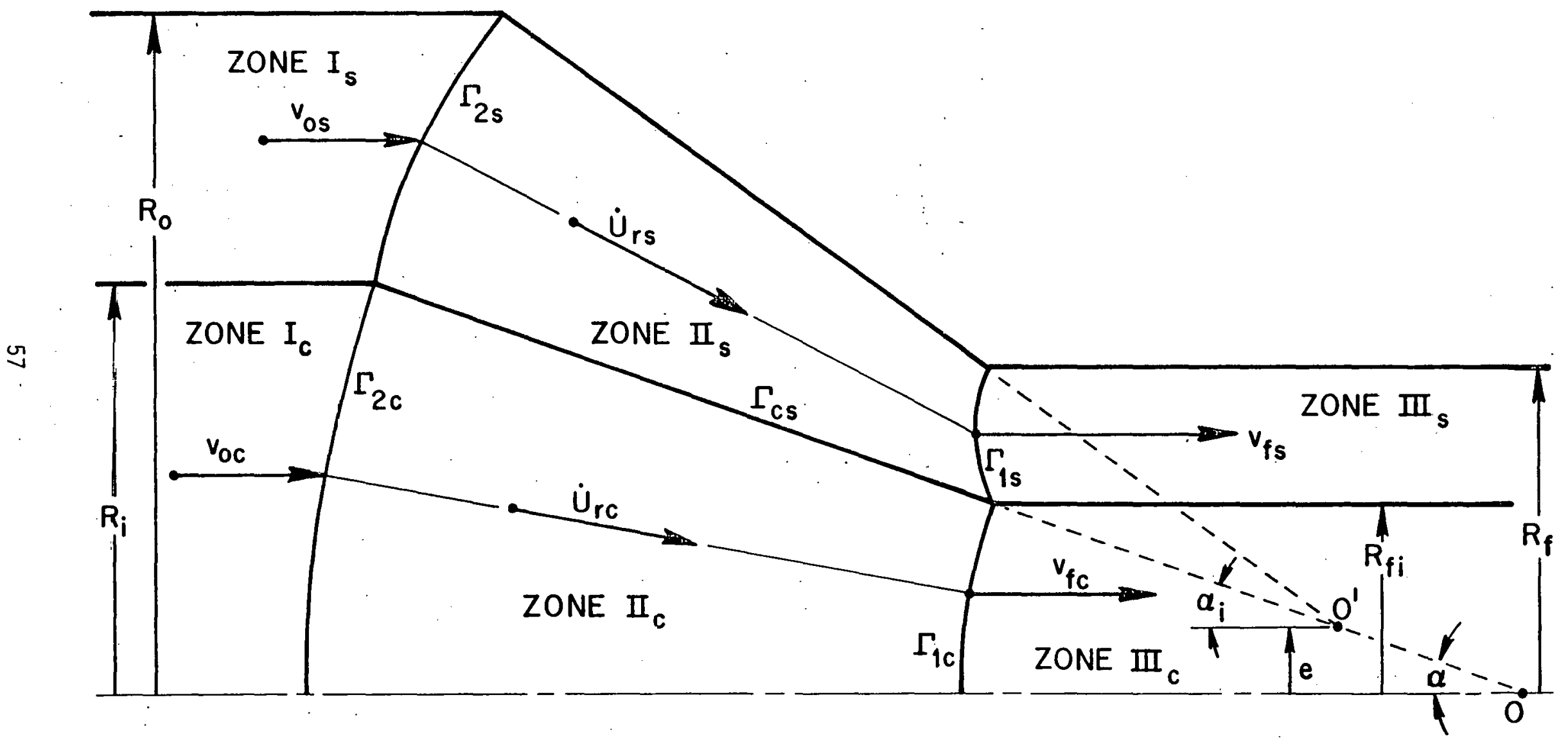

FIG.I2 COMBINED FLOW OF THE SLEEVE AND THE CORE. 


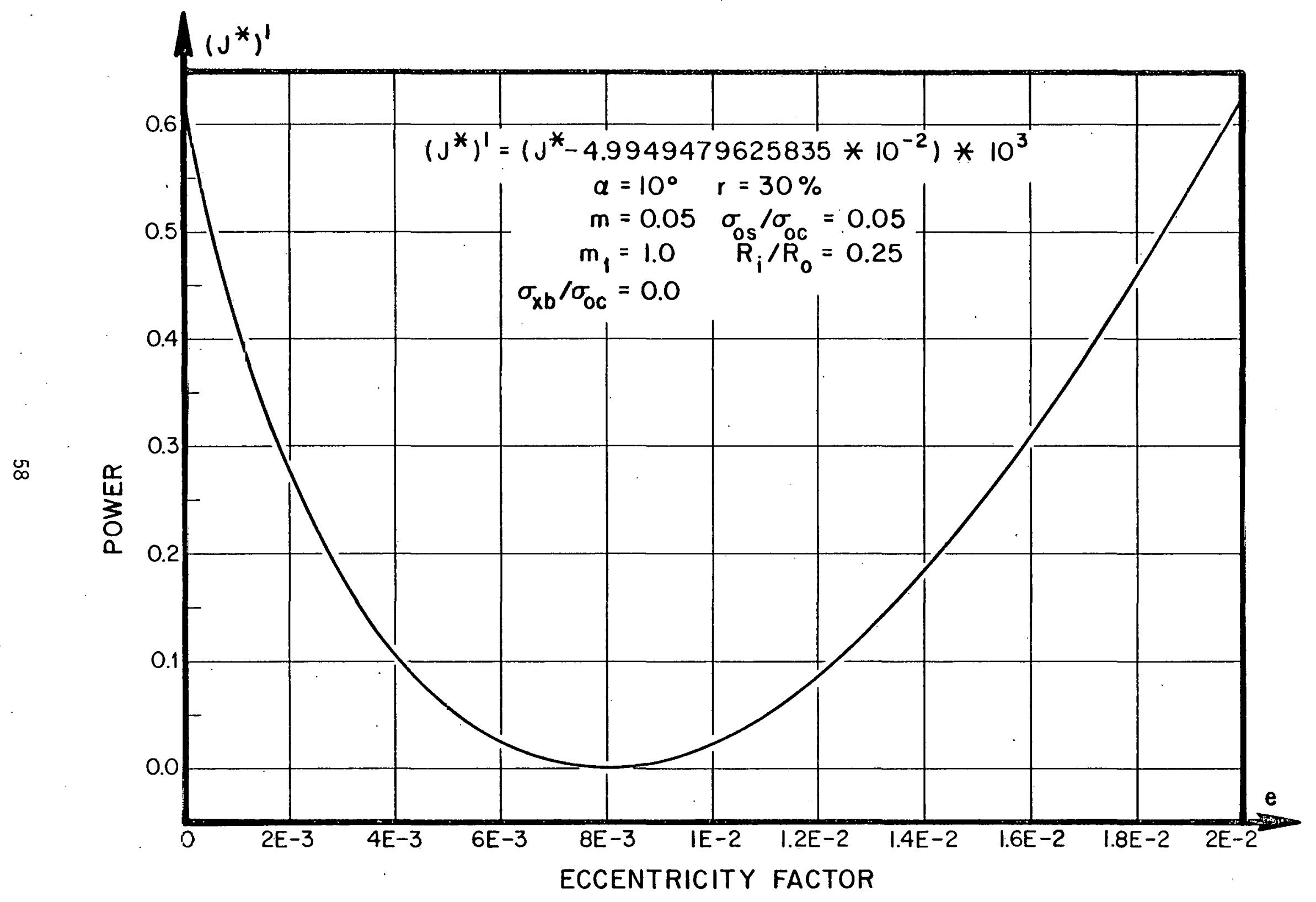

FIG. I3 TOTAL POWER VS. ECCENTRICITY FACTOR E WHEN DEVIATION FACTOR $\in$ IS ZERO. 


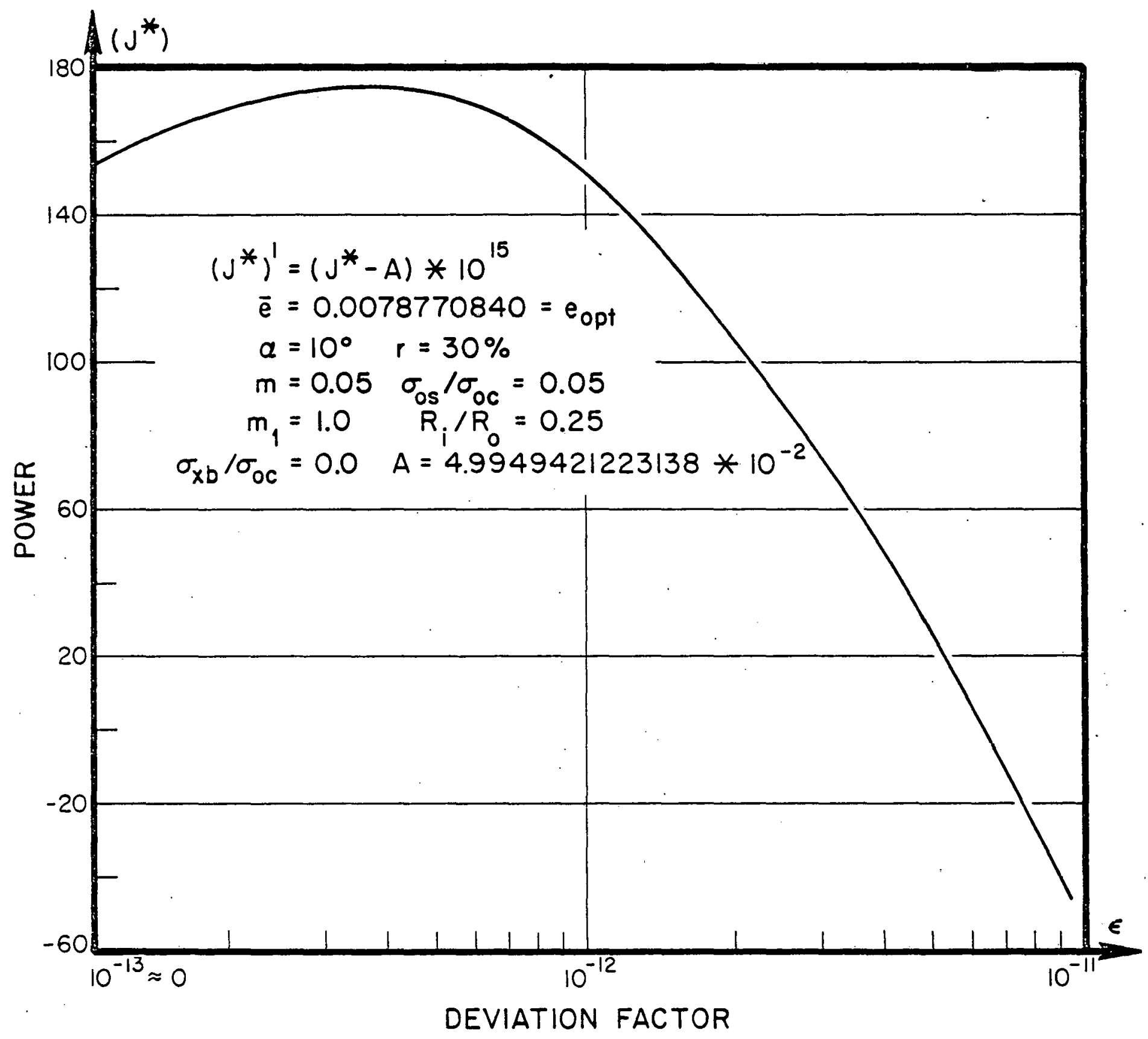

FIG.I4 TOTAL POWER VS. DEVIATION FACTOR $\epsilon$ $\left(A T e=e_{\text {opt }}\right)$ FOR PROPORTIONAL FLOW. 


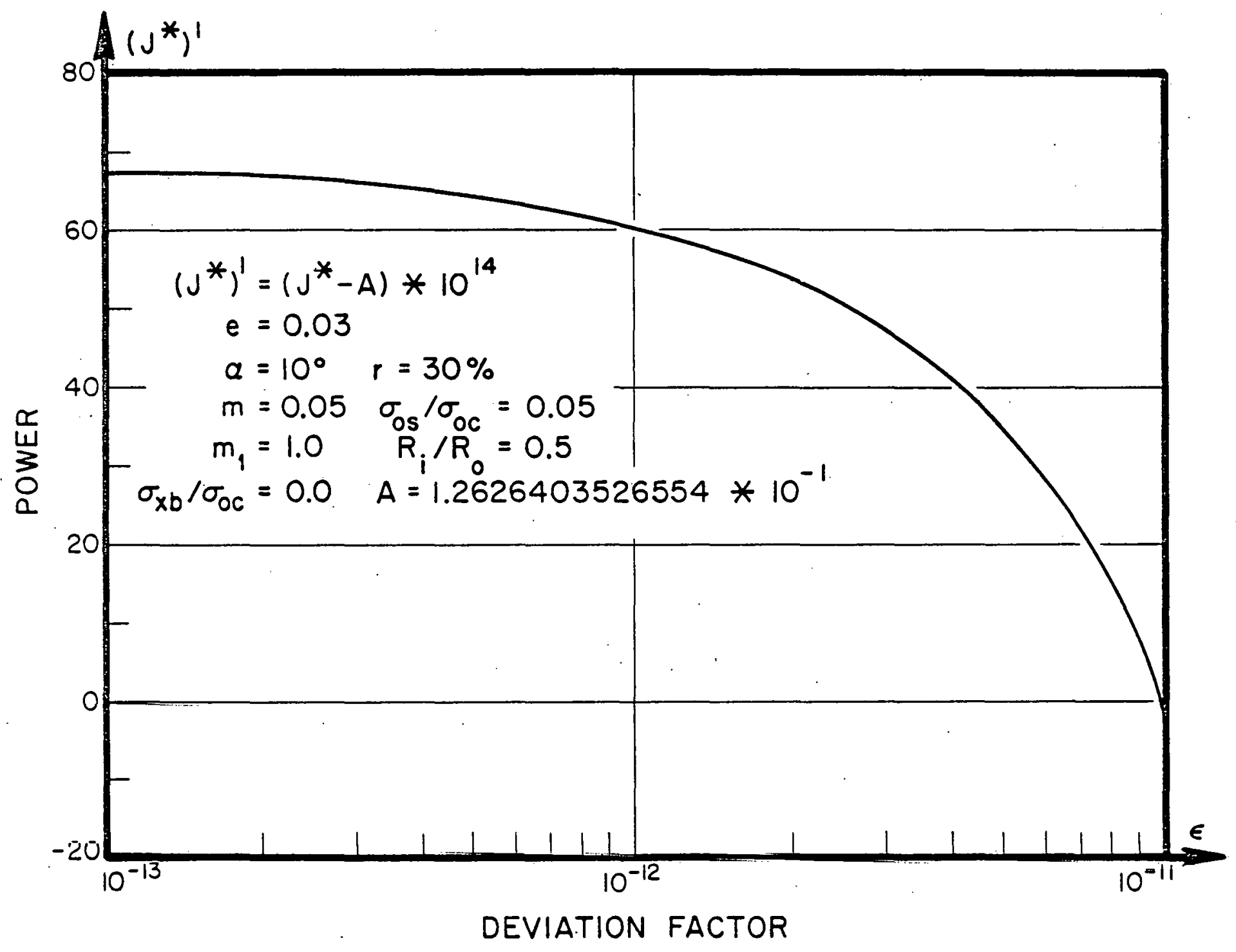

FIG.15a TOTAL POWER VS. DEVIATION FACTOR $\epsilon$ $\left(A T e=e_{\text {opt }}\right)$ FOR CORE FRACTURE. 


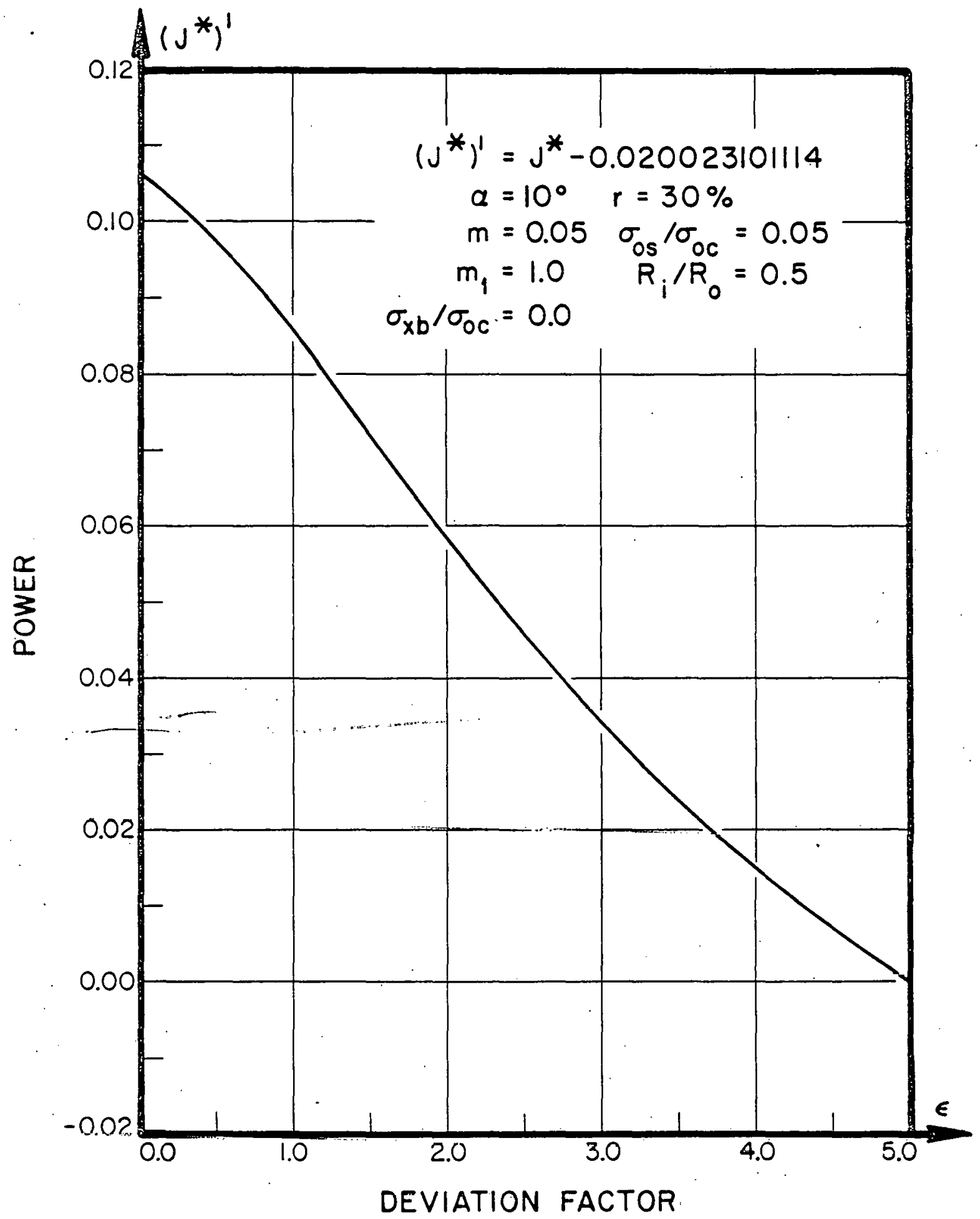

FIG.I5b TOTAL POWER VS. DEVIATION FACTOR $\epsilon$ $\left(A T e=e_{\text {opt }}\right)$ FOR CORE FRACTURE. 


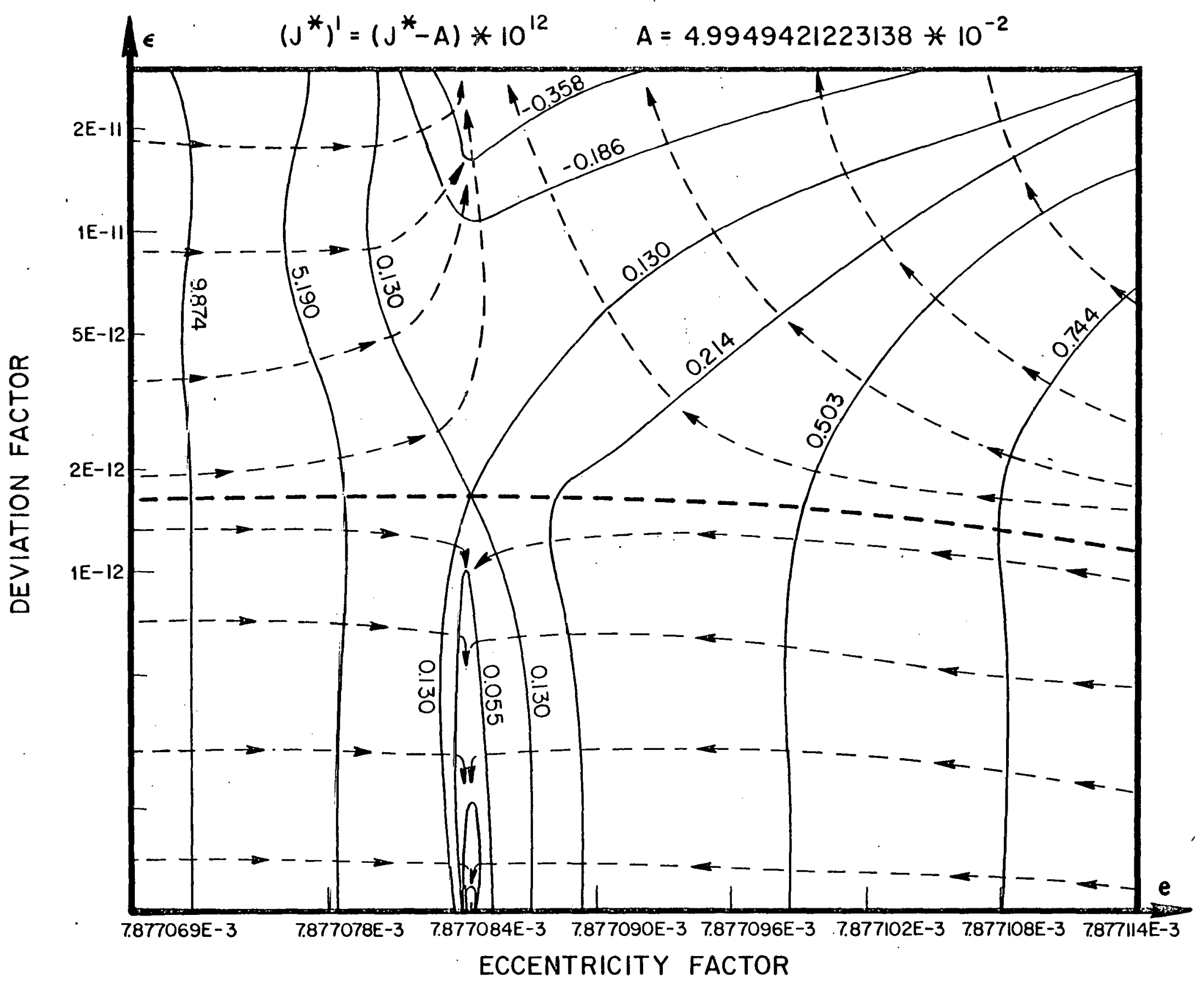

FIG.16 $\jmath^{*}$ CONTOUR FOR PROPORTIONAL FLOW. 


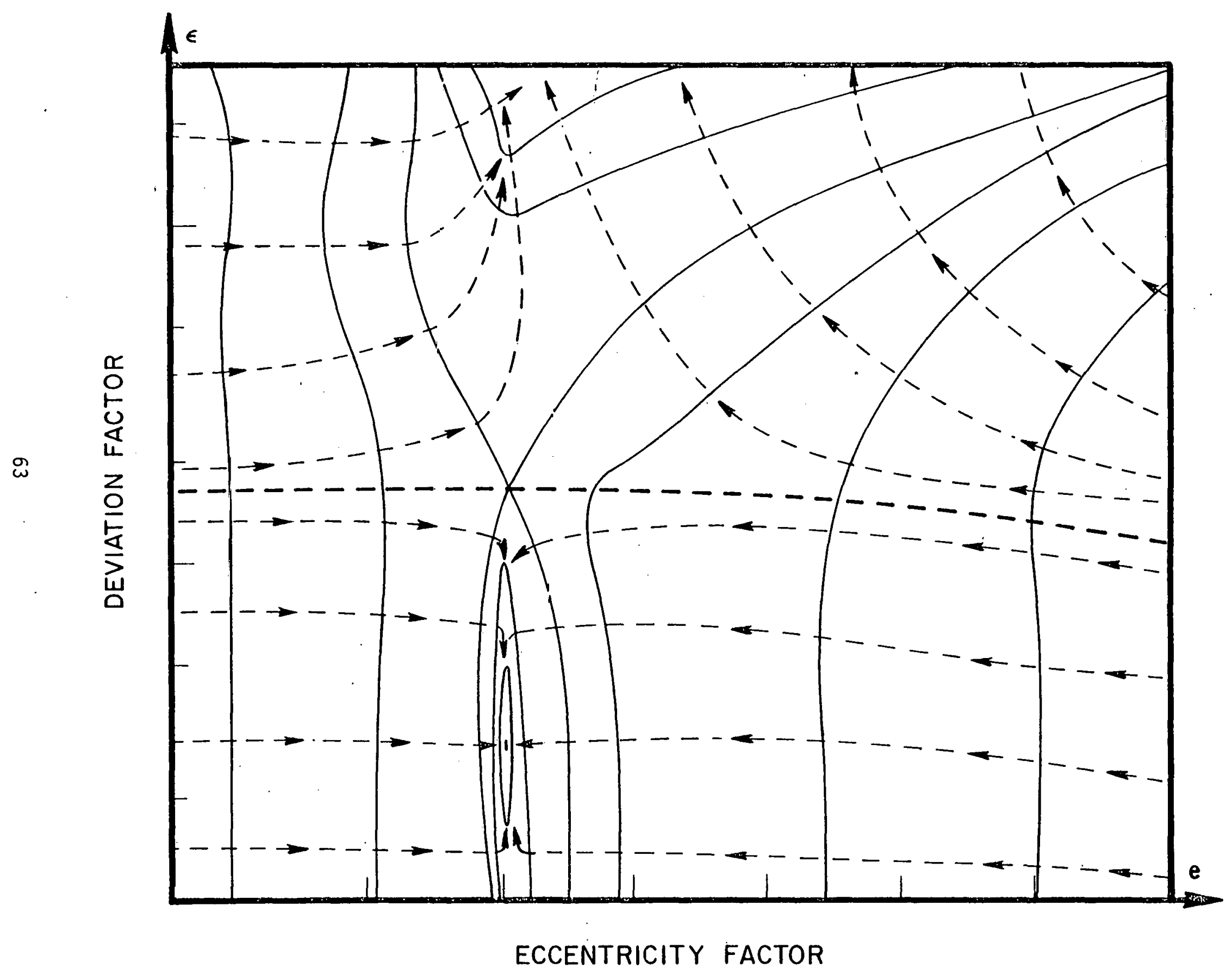

FIG.I7 $J^{*}$ CONTOUR FOR CORE FRACTURE. (HYPOTHETICAL) 


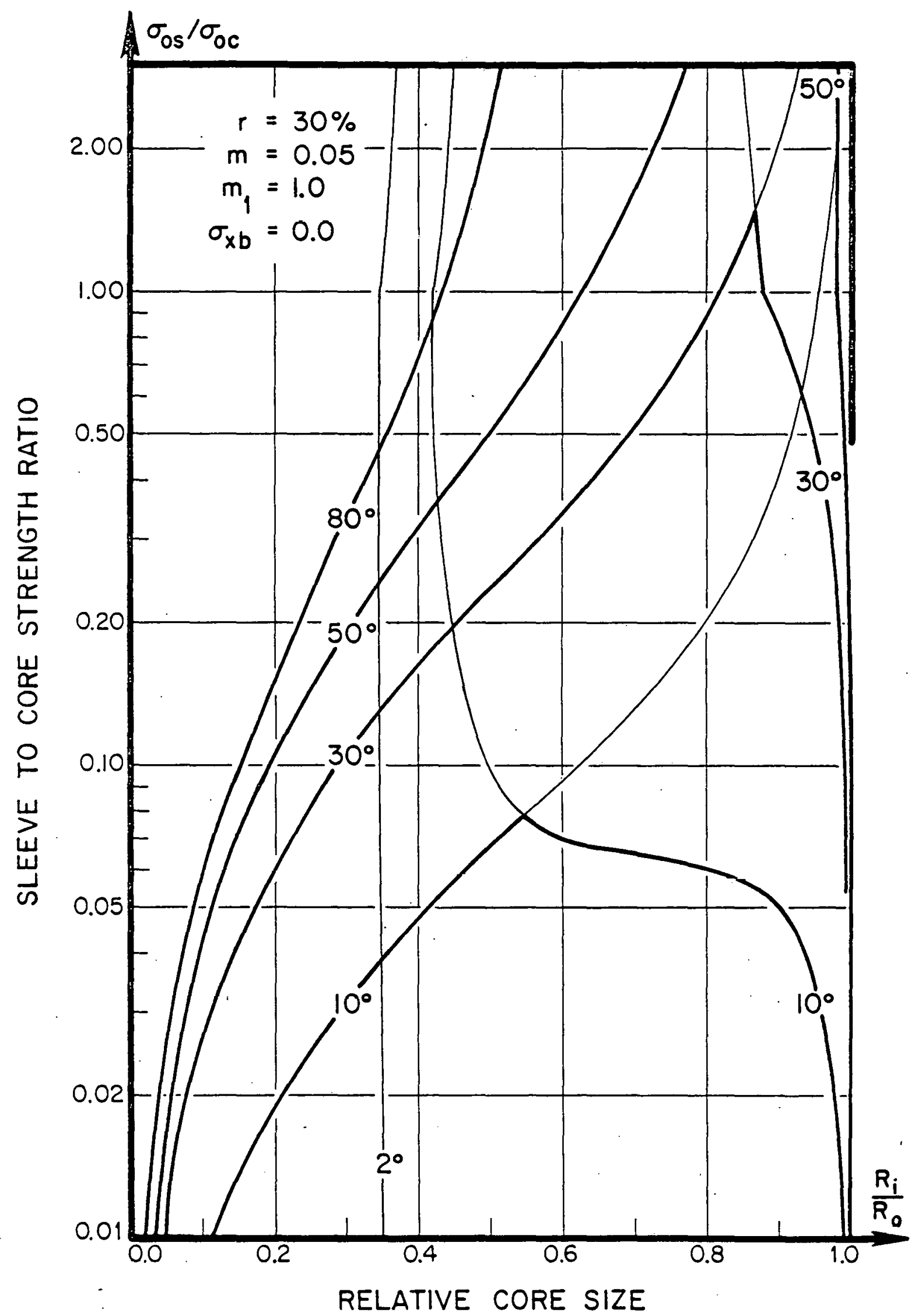

FIG.I8a CRITERION FOR CORE FRACTURE IN DRAWING. $\left(\sigma_{o s} / \sigma_{o c}\right.$ VS. $R_{i} / R_{0}$ WITH CHANGING a.) 


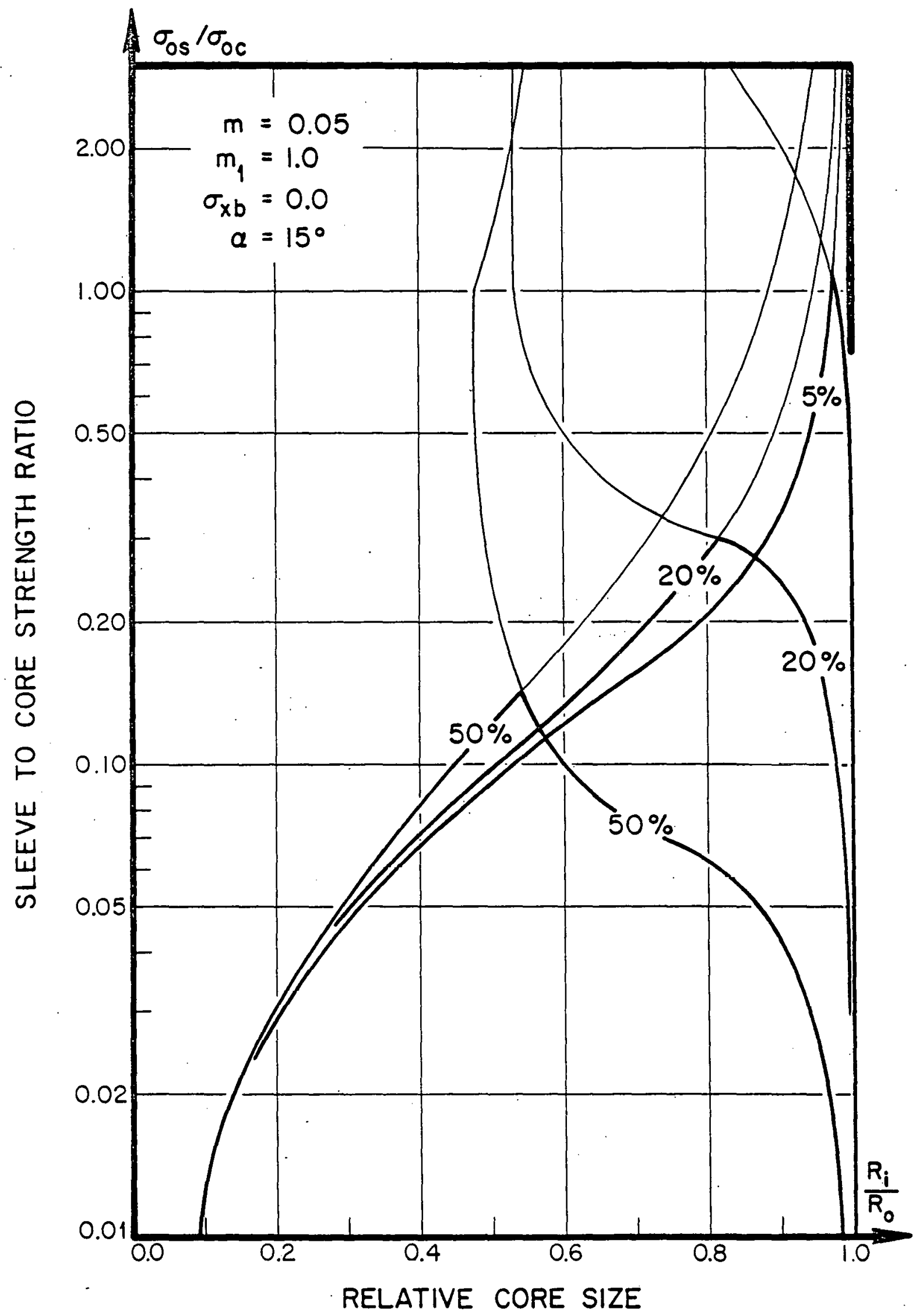

FIG.I8b CRITERION FOR CORE FRACTURE IN DRAWING. $\left(\sigma_{o s} / \sigma_{o c}\right.$ VS. $R_{i} / R_{o}$ WITH CHANGING $\left.r \%\right)$ 


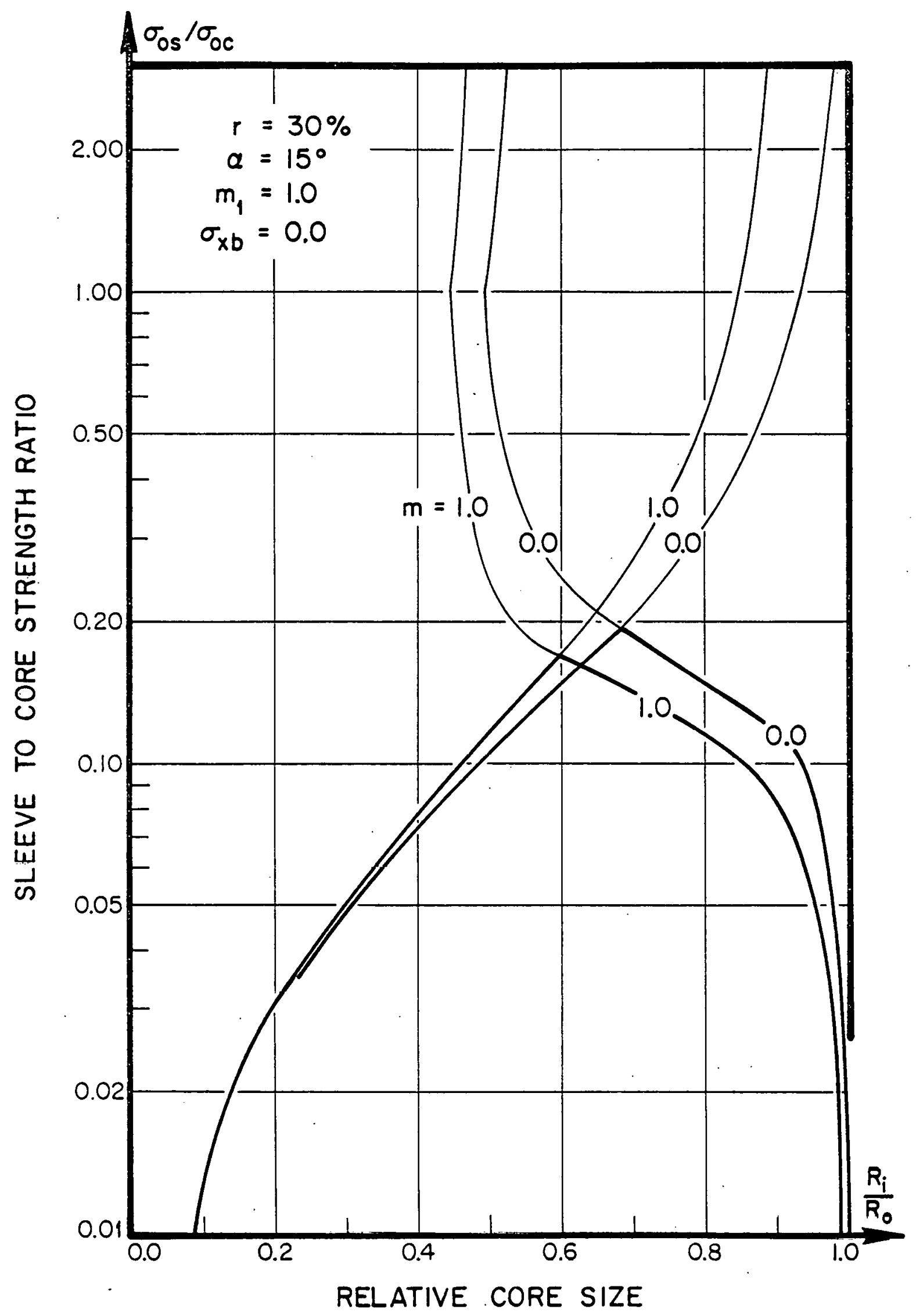

FIG.I8C CRITERION FOR CORE FRACTURE IN DRAWING. $\left(\sigma_{o s} / \sigma_{o c}\right.$ VS. $R_{i} / R_{0}$ WITH CHANGING m.) 


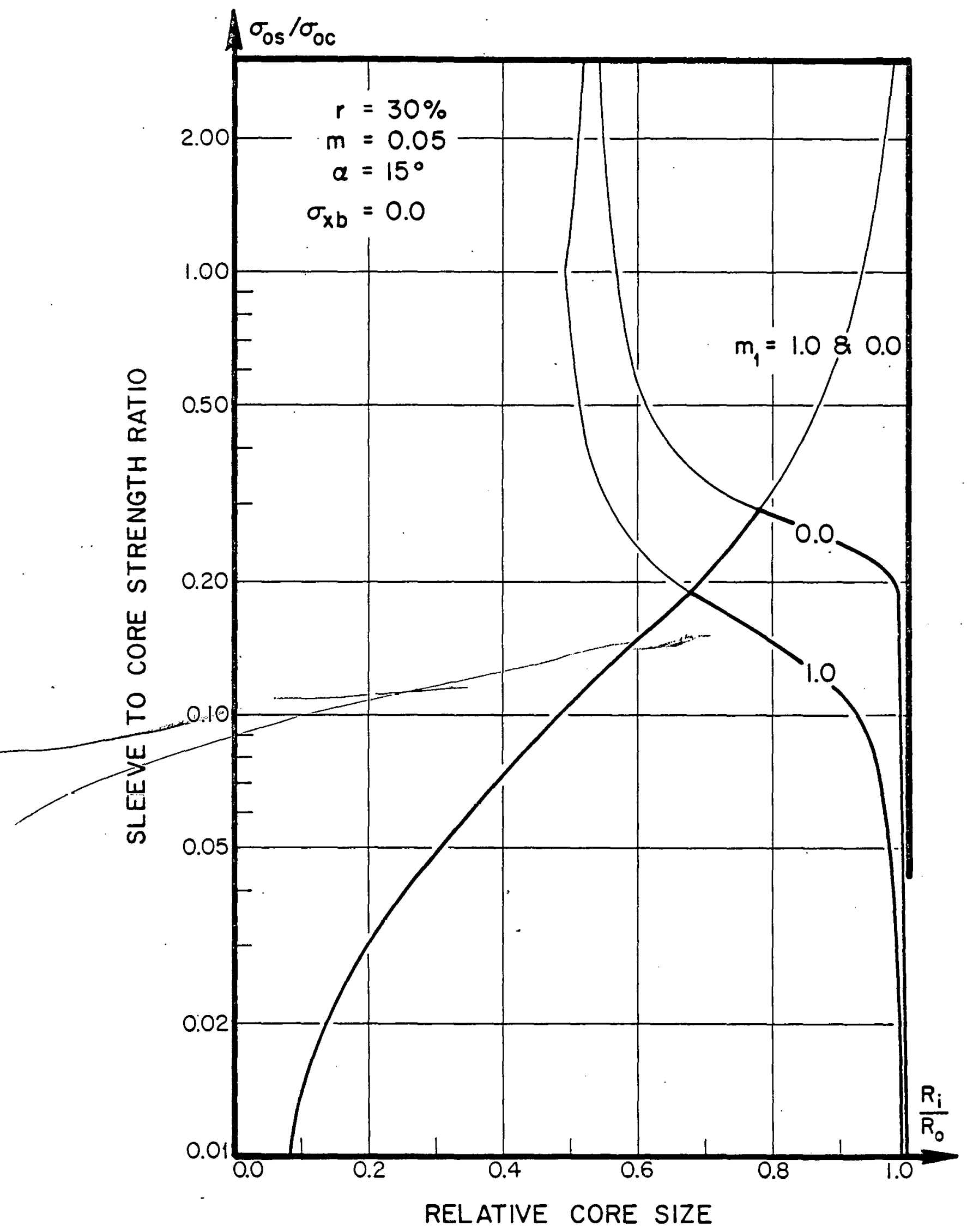

FIG.I8d CRITERION FOR CORE FRACTURE IN DRAWING. $\left(\sigma_{o s} / \sigma_{o c}\right.$ VS. $R_{i} / R_{0}$ WITH CHANGING $\left.m_{1}\right)$ 


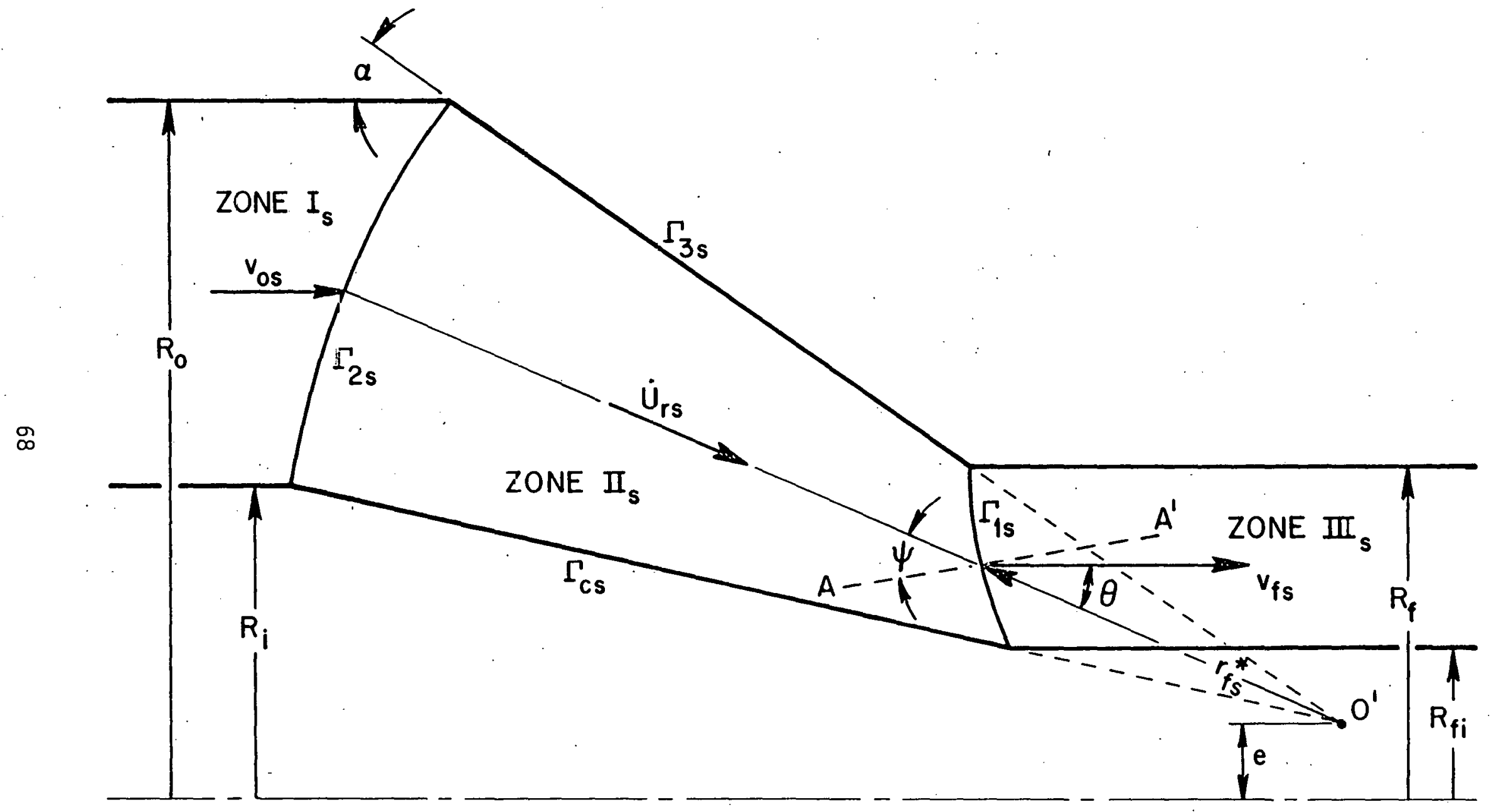

FIG.1 DETERMINATION OF THE SURFACE OF VELOCITY DISCONTINUITY AT THE EXIT SIDE OF THE SLEEVE. 


\section{APPENDIX A}

IS BULKY, CONTAINS ONLY BACKGROUND INFORMATION AND CAN BE FORWARDED ON REQUEST. 
APPENDIX B

Determination of the Radial Coordinate $r_{f s}^{\star}$

of the Surface of Discontinuity $\Gamma_{15}$. 
The principle of volume constancy, requires that the normal component of velocity across the surface of discontinuity $\Gamma_{7 \mathrm{~s}}$ must remain continuous. The radial distance $r_{f s}^{*}$ of this surface $\Gamma$ fs be found from the principle of volume constancy.

As shown in Fig. 1 , each plane AA, normal to the surface $\Gamma_{15}$, makes an angle $\psi$ with the radial direction. Thus, the velocity continuity of the normal component across $\Gamma_{1 s}$ surface dictates

$$
-\left(\dot{U}_{r s}\right)_{r=r_{f s}^{*}}: \cos \psi=v_{f s} \cos (\psi-\theta)
$$

where $\psi$ is defined by Eq. (46) as

$$
\psi=\tan ^{-1}\left(\frac{1}{r_{f}^{*}} \frac{d^{r} t s}{d \theta}\right)
$$

Substituting $\left(\dot{U}_{r s}\right)_{r=r_{f s}^{*}}^{*}$ of Eq. (5) and Eq. (46) into
$(66)$, leads to Eq. (66), leads to

$$
(\tan \theta) \frac{d r_{f s}^{\star}}{d \theta}+r_{f s}^{\star}-\frac{V_{o s}}{V_{f s}} \cdot \frac{r_{0 s}\left(r_{0 s} \sin \theta+e\right)}{\left(r_{f s}^{\star} \sin \theta+e\right)}=0
$$

Eq. (67) is a first order differential equation with the boundary condition

$$
\left(r_{f s}^{*}\right)_{\theta=\alpha}=\frac{R_{f}-e}{\sin \alpha}
$$

After lengthy manipulations, the solution of Eq. (67) is obtained as

$$
r_{f s}^{\star}(\theta, e, \varepsilon)=\frac{-e}{\sin \theta}+\frac{R_{0}}{\sin \theta}\left\{\frac{V_{o s}}{V_{f s}}\left[\left\langle\frac{\sin \theta}{\sin \alpha}+\frac{e}{R_{0}}\left(1-\frac{\sin \theta}{\sin \alpha}\right)\right\rangle^{2}-1\right]+\left(\frac{R_{f}}{R_{0}}\right)^{2}\right\}^{1 / 2}
$$




\section{APPENDIX}

ESTIMATION OF $\eta_{0}$ DUE TO INERTIA AND INTERCEPTION FOR LOW N $N_{S t}$ AND ZERO $N_{\text {Re, }, \text { : }}$

(A Case of Sinusoidal Wall Geometry and Perturbation Solution)

A calculation of the initial collection efficiency of a constricted tube with sinusoidal wall geometry, due to the combined effect of interception and particle inertia for low $N_{S t}\left(N_{S t}=0.001\right)$ with the flow field given by the perturbation solution, yields the following results:

\begin{tabular}{cccccc}
\hline$N_{R}$ & 0.05 & 0.02 & 0.0124 & 0.003 & 0.001 \\
\hline$\left\{\left(n_{0}\right)_{I, i}\right\}_{S}$ & 0.195 & 0.160 & 0.149 & 0.141 & 0.131 \\
\hline
\end{tabular}

Using linear regression for en $\left\{\left(n_{0}\right)_{1, i}\right\}$ variation with $\ln \left\{\mu_{R}\right\}$, one has

$$
\left\{\left(n_{0}\right)_{I, i}\right\}_{S}=(0.2302) N_{R}^{0.08243}
$$

